\title{
Global boundedness of a class of multilinear Fourier integral operators
}

\author{
Salvador Rodríguez-López ${ }^{1}$, David Rule ${ }^{2}$ and Wolfgang Staubach ${ }^{1,3}$ \\ ${ }^{1}$ Department of Mathematics, Stockholm University, SE-106 91 Stockholm, Sweden; \\ E-mail: s.rodriguez-lopez@math.su.se. \\ ${ }^{2}$ Department of Mathematics, Linköping University, SE-581 83 Linköping, Sweden; E-mail: david.rule@liu.se. \\ ${ }^{3}$ Department of Mathematics, Uppsala University, SE-751 06 Uppsala, Sweden; E-mail: wulf@math.uu.se.
}

Received: 15 May 2020; Revised: 15 January 2021; Accepted: 27 January 2021

2020 Mathematics Subject Classification: Primary - 35S30, 42B20, 30H10; Secondary - 30H35, 42B25, 42B35

\begin{abstract}
We establish the global regularity of multilinear Fourier integral operators that are associated to nonlinear wave equations on products of $L^{p}$ spaces by proving endpoint boundedness on suitable product spaces containing combinations of the local Hardy space, the local BMO and the $L^{2}$ spaces.
\end{abstract}

\section{Introduction}

This paper deals with the global boundedness of a class of multilinear Fourier integral operators that appear frequently in connection to nonlinear wave equations. To illustrate this, fix a smooth, compactly supported multilinear symbol $m$ on $\mathbb{R}^{n N}$. Let $T_{m}$ denote the multilinear paraproduct

$$
T_{m}\left(f_{1}, \ldots, f_{N}\right)(x):=\int_{\mathbb{R}^{n N}} m(\Xi) \prod_{j=1}^{N}\left(\widehat{f}_{j}\left(\xi_{j}\right) e^{i x \cdot \xi_{j}}\right) \mathrm{d} \Xi,
$$

where $x \in \mathbb{R}^{n}, \xi_{j} \in \mathbb{R}^{n}(j=1, \ldots, N), \Xi=\left(\xi_{1}, \ldots, \xi_{N}\right) \in \mathbb{R}^{n N}$ and

$$
\widehat{f}(\xi)=\int_{\mathbb{R}^{n}} f(x) e^{-i x \cdot \xi} \mathrm{d} x,
$$

is the Fourier transform of $f$.

Furthermore, set

$$
\sqrt{-\Delta} f(x)=\int_{\mathbb{R}^{n}}|\xi| \widehat{f}(\xi) \cdot e^{i x \cdot \xi} \mathrm{d} \xi
$$

where $\mathrm{d} \xi$ denotes the normalised Lebesgue measure $\mathrm{d} \xi /(2 \pi)^{n}$. Consider now the wave equations

$$
\left\{\begin{array} { l } 
{ i \partial _ { t } u + \sqrt { - \Delta } u = T _ { m } ( v _ { 1 } , \ldots , v _ { N } ) } \\
{ i \partial _ { t } v _ { k } + \sqrt { - \Delta } v _ { k } = 0 , \quad k = 1 , \ldots , N , }
\end{array} \quad \text { with } \quad \left\{\begin{array}{l}
u(0, x)=0 \\
v_{k}(0, x)=f_{k}(x), k=1, \ldots, N
\end{array}\right.\right.
$$

The functions $u$ and $v_{k}$ are complex valued, and each $f_{k}$ maps $\mathbb{R}^{n}$ to $\mathbb{C}$. The system of equations (2) is used to study the nonlinear interaction of free waves, as a first step towards understanding a nonlinear

(C) The Author(s), 2021. Published by Cambridge University Press. This is an Open Access article, distributed under the terms of the Creative Commons Attribution licence (http://creativecommons.org/licenses/by/4.0/), which permits unrestricted re-use, distribution, and reproduction in any medium, provided the original work is properly cited. 
wave equation $i \partial_{t} u+\sqrt{-\Delta} u=F(u)$, with a suitable nonlinearity. The main question here is, given $f_{k}$ in some function spaces, how does $u$ behave in some other suitable function space? In order to answer this question, one uses the Duhamel formula to represent the solution $u$ as

$$
u(t, x)=\int_{0}^{t} \int_{\mathbb{R}^{n N}} m(\Xi) \prod_{j=1}^{N}\left(\widehat{f}_{j}\left(\xi_{j}\right) e^{i x \cdot \xi_{j}+i s\left|\xi_{j}\right|}\right) e^{i(t-s)\left|\xi_{1}+\cdots+\xi_{N}\right|} \mathrm{d} \Xi \mathrm{d} s .
$$

The inner integral in equation (3) is precisely of the form of the operators whose boundedness are studied in this paper. This is of course along the lines of the far-reaching method of space-time resonances which was introduced by Germain, Masmoudi and Shatah and which they explored and applied to nonlinear partial differential equations in [8], and which Bernicot and Germain also expored in [1]. In our case, we are ignoring the effect of the integral in $s$, which amounts to ignoring the effects of the time resonance.

Motivated by equation (3), we study multilinear Fourier integral operators (abbreviated multilinear FIOs) of the form

$$
T_{\sigma}^{\Phi}\left(f_{1}, \ldots, f_{N}\right)(x)=\int_{\mathbb{R}^{n N}} \sigma(x, \Xi) \prod_{j=1}^{N}\left(\widehat{f}_{j}\left(\xi_{j}\right) e^{i x \cdot \xi_{j}}\right) e^{i \Phi(\Xi)} \mathrm{d} \Xi
$$

where $\sigma$ is an amplitude and

$$
\Phi(\Xi)=\varphi_{0}\left(\xi_{1}+\cdots+\xi_{N}\right)+\sum_{j=1}^{N} \varphi_{j}\left(\xi_{j}\right)
$$

is a combination of phase functions $\varphi_{j}(j=0,1, \ldots, N)$. Here the terms 'amplitude' and 'phase function' are defined as follows:

Definition 1.1. For integers $n, N \geqslant 1$ and $m \in \mathbb{R}$, the set of (multilinear) amplitudes $S^{m}(n, N)$ is the set of functions $\sigma \in \mathcal{C}^{\infty}\left(\mathbb{R}^{n} \times \mathbb{R}^{n N}\right)$ that satisfy

$$
\left|\partial_{\Xi}^{\alpha} \partial_{x}^{\beta} \sigma(x, \Xi)\right| \leqslant C_{\alpha, \beta}\langle\Xi\rangle^{m-|\alpha|},
$$

for all multi-indices $\alpha$ and $\beta$. Here and in what follows,

$$
\langle\Xi\rangle=\left(1+\sum_{j=1}^{N}\left|\xi_{j}\right|^{2}\right)^{1 / 2} \quad \text { for } \Xi=\left(\xi_{1}, \ldots, \xi_{N}\right) \in \mathbb{R}^{n N} \text { with } \xi_{j} \in \mathbb{R}^{n}, \mathrm{j}=1, \ldots, \mathrm{N} \text {. }
$$

The parameter $m$ is referred to as the order or decay of the amplitude.

Remark 1.2. The terminology 'multilinear FIOs' that is used in this paper stems from the fact that for the linear (half-)wave equation $i \partial_{t} u+\sqrt{-\Delta} u=0$ with initial data $u(0, x)=f(x)$, there is a representation of the solution in the form of an FIO acting on $f$, with the phase function $x \cdot \xi+|\xi|$ which is positively homogeneous of degree 1 in $\xi$. This is one of the historical sources for the theory of Fourier integral operators. Using this analogy in connection to the nonlinear system of wave equations (2), we believe that the nomenclature 'multilinear FIOs' is justified. However, one should note that for general multilinear FIOs, the phase functions are of the form $\Phi(x, \Xi)$, in which the linear phases $x \cdot \sum_{j=1}^{N} \xi_{j}$ are subsumed, and one assumes that $\Phi(x, \Xi)$ is positively homogeneous of degree 1 in $\Xi$ and also satisfies some suitable geometric conditions. Although the investigation that is carried out in this paper is not suited for these more general multilinear FIOs, our motivation is based on questions in the theory of nonlinear wave equations connected to the system (2), for which we are able to carry out a fairly complete analysis. 
Definition 1.3. A function $\varphi: \mathbb{R}^{n} \rightarrow \mathbb{R}$ which belongs to $\mathcal{C}^{\infty}\left(\mathbb{R}^{n} \backslash\{0\}\right)$ and is positively homogeneous of degree 1 - that is, it satisfies $\varphi(t \xi)=t \varphi(\xi)$ for all $\xi \in \mathbb{R}^{n}$ and all $t>0$ - is called a phase function (or phase).

In order to state the main result of this paper - Theorem 1.4 - we define

$$
X^{p}:= \begin{cases}h^{p} & \text { if } p \leqslant 1, \\ L^{p} & \text { if } 1<p<\infty, \\ \text { bmo } & \text { if } p=\infty,\end{cases}
$$

where $L^{p}$ is the usual Lebesgue space, $h^{p}$ is the local Hardy space defined in Definition 2.2 and bmo is the dual space of $h^{1}$. We remind the reader that $L^{p}$ and $h^{p}$ coincide when $1<p<\infty$.

Theorem 1.4. Given integers $n, N \geqslant 2$ and exponents $p_{j} \in[1, \infty](j=0, \ldots, N)$ which satisfy

$$
\frac{1}{p_{0}}=\sum_{j=1}^{N} \frac{1}{p_{j}}
$$

suppose that

$$
m \leqslant-(n-1)\left(\sum_{j=1}^{N}\left|\frac{1}{p_{j}}-\frac{1}{2}\right|+\left|\frac{1}{p_{0}}-\frac{1}{2}\right|\right) .
$$

Then if $\sigma \in S^{m}(n, N)$ and $\Phi$ is of the form in equation (5), with each phase $\varphi_{j}$ as in Definition 1.3 $(j=0,1, \ldots, N)$, then the multilinear operator $T_{\sigma}^{\Phi}$ initially defined by equation (4) for $f_{1}, \ldots, f_{N} \in \mathcal{S}$ (the Schwartz class) extends to a bounded multilinear operator from $X^{p_{1}} \times \cdots \times X^{p_{N}}$ to $X^{p_{0}}$.

We can compare this result with earlier work [21] of ours. The first novelty of the present result is its global nature, in the sense that it doesn't require the amplitudes $\sigma(x, \Xi)$ to be compactly supported in the spatial variable $x$. Indeed, this paper establishes the first global results to date for multilinear (or even bilinear) Fourier integral operators. The second novelty is that we allow a component of the phase function of $T_{\sigma}^{\Phi}$ to depend on a mix of the variables $\xi_{1}, \ldots, \xi_{N}$ in a way that is dictated by the nonlinear wave equation applications, as already demonstrated. In [21], the phase $\varphi_{0}$ was not present (that is, it was identically zero). The third novelty is that the results are proved for multilinear and not just bilinear operators, as was the case in [21]. There is also a difference in the function spaces considered. In [21], the endpoint function spaces whose products formed the domain of the operator were the real Hardy space $H^{1}$ and its dual BMO, whereas here we consider the larger function space $h^{1}$ and its dual bmo. Although $h^{1}$ was used as an important technical tool in [21], here it takes centre stage. In [21] the restriction $p_{0} \geqslant 1$ was not imposed and the target space $X^{p_{0}}$ was $L^{p_{0}}$ even for $p_{0} \leqslant 1$. The natural improvement to consider here would be $X^{p_{0}}$ equal to the local Hardy space $h^{p_{0}}$ when $p_{0} \leqslant 1$, but this possibility is reserved for a forthcoming paper. In the present paper we provide an example showing that both Theorem 1.4 and the main result in [21] are sharp in the case where $N=2$ and $p_{1}=p_{2}=2$.

In proving our multilinear boundedness results, it behooved us also to prove the global regularity of linear Fourier integral operators on local Hardy spaces $h^{p}$ and local spaces of functions of bounded mean oscillations bmo. The local version of this result is stated in the work of Seeger, Sogge and Stein [23] (the case where $1<p<\infty$ ) and of Peloso and Secco [19] (the case where $0<p<1$ ), but is not enough for our purposes. Indeed, it is not enough even if the amplitude $\sigma(x, \Xi)$ is assumed to have compact $x$-support. This is because the introduction of the mixed phase $\varphi_{0}\left(\xi_{1}+\cdots+\xi_{N}\right)$ leads to the appearance of global Fourier integral operators in the subsequent high-frequency decomposition of the operator, so the more complicated phase appears to necessitate the study of global regularity of linear Fourier integral operators. The global linear regularity is proved by a transference procedure due to Ruzhansky and Sugimoto [22]. We prove this regularity for exponents $p>n /(n+1)$, which differs from 
the range in the local case, where $p$ can take any positive value. However, we prove this is the sharp range in the global setting. In the present paper we only make use of this linear result in the case where $p=1$, but the full range of exponents will come into play in the forthcoming paper already mentioned.

Beyond the need to understand global Fourier integral operators, the presence of the mixed phase $\varphi_{0}\left(\xi_{1}+\cdots+\xi_{N}\right)$ leads to other difficulties. The underlying cause of these difficulties is the failure of the commutator techniques which were an essential ingredient in [21]. To successfully apply such techniques in this context would require better control of the commutator between a linear Fourier integral operator and a multiplication operator (that which is denoted $M_{\mathfrak{m}}$ in Section 7) than seems reasonable to expect. Instead, we succeed in decomposing the operators into a sum of the constant coefficient operators (that is, the case $\sigma(x, \Xi)$ does not depend on $x$, which corresponds to $M_{\mathfrak{m}}$ being the identity operator). This requires at times careful control of the Carleson measure generated by a bmo function.

The multilinear results of this paper are then achieved through the following steps. First we identify the endpoints that are needed for the complex interpolation which leads to the regularity of multilinear Fourier integral operators on products of $L^{p}$ spaces. Thereafter we make a multilinear phase space analysis to divide the operator according to various frequency supports of the amplitude. This creates a number of cases with their associated difficulties, which are dealt with in accordance to the form of the endpoints in question. Finally, complex interpolation yields the main result.

The paper is organised as follows. In Section 2 we recall some definitions, as well as results from linear and multilinear harmonic and microlocal analysis. In Section 3 we prove the global $h^{p}$ and bmo regularity of Fourier integral operators using, among other things, Ruzhansky and Sugimoto's globalisation procedure. In Section 4 we show that the global $h^{p}$ and bmo regularity result and Theorem 1.4 are in some sense sharp. Section 5 is devoted to finding the so-called endpoints for which complex interpolation would provide the final regularity result for multilinear Fourier integral operators. Finally, in Sections 7, 8 and 9 we systematically analyse all the endpoint cases for various frequency localisations.

\section{Definitions and preliminaries}

In this section, we will collect all the definitions that will be used throughout this paper. We also state some useful results from both harmonic and microlocal analysis which will be used in the proofs of our results.

The proof of Theorem 1.4 builds upon the corresponding linear results. Indeed, as mentioned in Section 1, the proof we present requires new linear boundedness results. We begin by recalling the definitions of the linear versions of the main objects of study in this paper. The multilinear amplitudes defined in Definition 1.1 reduce to the classical Hörmander classes $S^{m}$ of amplitudes (or symbols) in the case where $N=1$ - that is to say, $S^{m}=S^{m}(n, 1)$. The same is true of linear Fourier integral operators: they are the special case of equation (4) when $N=1$, so in that case we write

$$
T_{a}^{\varphi} f(x):=\int_{\mathbb{R}^{n}} e^{i x \cdot \xi+i \varphi(\xi)} a(x, \xi) \widehat{f}(\xi) \mathrm{d} \xi,
$$

for a given amplitude $a \in S^{m}$ and phase function $\varphi$. Such an operator is called a pseudodifferential operator under the further restriction that $\varphi \equiv 0$. In this case it is useful to introduce slightly different (although widely used) notation: for $a \in S^{m}$ we define a (linear) pseudodifferential operator to be the operator

$$
a(x, D) f(x):=\int_{\mathbb{R}^{n}} e^{i x \cdot \xi} a(x, \xi) \widehat{f}(\xi) \mathrm{d} \xi,
$$

which, as is the case for all FIOs, is a priori defined on the Schwartz class $\delta\left(\mathbb{R}^{n}\right)$. The terminology symbol is typically used in connection with pseudodifferential operators, and amplitude with Fourier integral operators.

We will denote constants which can be determined by known parameters in a given situation, but whose values are not crucial to the problem at hand, by $C$ or $c$, sometimes adding a subscript - for 
example, $c_{\alpha}$ - to emphasise a dependency on a given parameter (here, $\alpha$ ). Such parameters are those which determine function spaces, such as $p$ or $m$, the dimension $n$ of the underlying Euclidean space and the constants connected to the seminorms of various amplitudes or phase functions. The value of the constants may differ from line to line, but in each instance could be estimated if necessary. We also write $a \lesssim b$ as shorthand for $a \leqslant C b$ and $a \approx b$ when $a \lesssim b$ and $b \lesssim a$. By

$$
B(x, r):=\left\{y \in \mathbb{R}^{n}:|y-x|<r\right\}
$$

we denote the open ball of radius $r>0$ centred at $x \in \mathbb{R}^{n}$.

The following partition of unity is a standard tool in harmonic analysis and is even used to define the function spaces that we are concerned with.

Definition 2.1. Let $\psi_{0} \in \mathcal{C}_{c}^{\infty}\left(\mathbb{R}^{n}\right)$ be equal to 1 on $B(0,1)$ and be supported in $B(0,2)$. We define

$$
\psi_{j}(\xi):=\psi_{0}\left(2^{-j} \xi\right)-\psi_{0}\left(2^{-(j-1)} \xi\right)
$$

for integers $j \geqslant 1$. Then one has the following Littlewood-Paley partition of unity:

$$
\sum_{j=0}^{\infty} \psi_{j}(\xi)=1 \quad \text { for all } \xi \in \mathbb{R}^{n} .
$$

With the help of the Littlewood-Paley partition of unity we define local Hardy spaces first introduced by Goldberg [11].

Definition 2.2. For each $0<p<\infty$, the following characterisations of the local Hardy space $h^{p}\left(\mathbb{R}^{n}\right)$ are equivalent (see, for example, [26]):

(i) The set of tempered distributions $f \in \mathcal{S}^{\prime}\left(\mathbb{R}^{n}\right)$ such that

$$
\|f\|_{h^{p}\left(\mathbb{R}^{n}\right)}^{[1]}:=\left(\int \sup _{0<t<1}\left|\psi_{0}(t D) f(x)\right|^{p} \mathrm{~d} x\right)^{\frac{1}{p}}<\infty .
$$

(ii) The set of all $f \in \mathcal{S}^{\prime}\left(\mathbb{R}^{n}\right)$ such that

$$
\|f\|_{h^{p}\left(\mathbb{R}^{n}\right)}^{[2]}:=\left\|\left(\sum_{j=0}^{\infty}\left|\psi_{j}(D) f\right|^{2}\right)^{\frac{1}{2}}\right\|_{L^{p}\left(\mathbb{R}^{n}\right)}<\infty .
$$

(iii) The set of all $f \in \mathcal{S}^{\prime}\left(\mathbb{R}^{n}\right)$ for which there exist a sequence $\left(\lambda_{j}\right)_{j=1}^{\infty}$ of numbers and a sequence $\left(a_{j}\right)_{j=1}^{\infty}$ of $\left(h^{p}, M\right)$-atoms (abbreviated $h^{p}$-atoms) such that

$$
f=\sum_{j} \lambda_{j} a_{j}
$$

and

$$
\|f\|_{h^{p}\left(\mathbb{R}^{n}\right)}^{[3]}:=\inf \left(\sum_{j}\left|\lambda_{j}\right|^{p}\right)^{1 / p}<\infty
$$

where

$$
M \geqslant\left\lfloor n\left(\frac{1}{p}-1\right)_{+}\right\rfloor,
$$


with $\lfloor x\rfloor$ denoting the integer part of $x$. A function $a$ is called an $\left(h^{p}, M\right)$-atom if for some $x_{0} \in \mathbb{R}^{n}$ and $r>0$, the following three conditions are satisfied:

(a) $\operatorname{supp} a \subseteq B\left(x_{0}, r\right)$,

(b) $|a(x)| \leqslant\left|B\left(x_{0}, r\right)\right|^{-\frac{1}{p}}$ and

(c) if $r \leqslant 1$ and $|\alpha| \leqslant M$, then

$$
\int_{\mathbb{R}^{n}} x^{\alpha} a(x) \mathrm{d} x=0
$$

(iv) The set of all $f \in \mathcal{S}^{\prime}\left(\mathbb{R}^{n}\right)$ such that

$$
\|f\|_{h^{p}}^{[4]}:=\left\|\psi_{0}(D) f\right\|_{L^{p}}+\sum_{M \leqslant|\alpha| \leqslant M+1} \sup _{0<\varepsilon \leqslant 1}\left\|r_{\varepsilon}^{\alpha}(D) f\right\|_{L^{p}}<\infty
$$

where $M$ is as in (iii) and

$$
r_{\varepsilon}^{\alpha}(\xi)=\psi_{0}(\varepsilon \xi) \prod_{i=1}^{n}\left(\frac{\xi_{i}}{|\xi|}\right)^{\alpha_{i}}\left(1-\psi_{0}(\xi)\right)^{\alpha_{i}}
$$

(v) The set of all $f \in \mathcal{S}^{\prime}\left(\mathbb{R}^{n}\right)$ such that

$$
\|f\|_{h^{p}\left(\mathbb{R}^{n}\right)}^{[5]}:=\left(\int \sup _{0<t<1} \sup _{|x-y|<t}\left|\psi_{0}(t D) f(y)\right|^{p} \mathrm{~d} x\right)^{\frac{1}{p}}<\infty .
$$

Moreover all the norms here are equivalent - that is,

$$
\|f\|_{h^{p}\left(\mathbb{R}^{n}\right)}^{[1]} \approx\|f\|_{h^{p}\left(\mathbb{R}^{n}\right)}^{[2]} \approx\|f\|_{h^{p}\left(\mathbb{R}^{n}\right)}^{[3]} \approx\|f\|_{h^{p}\left(\mathbb{R}^{n}\right)}^{[4]} \approx\|f\|_{h^{p}\left(\mathbb{R}^{n}\right)}^{[5]},
$$

with implicit constants that depend only on the dimension $n$ and the choice of $\psi_{0}$ in the Littlewood-Paley decomposition - so we simply write $\|f\|_{h^{p}\left(\mathbb{R}^{n}\right)}$ for all of them.

It is also shown in [11] that a function $f$ belongs to the local Hardy space $h^{1}$ if and only if $f \in L^{1}$ and $\mathfrak{R}_{j}\left(\left(1-\psi_{0}\right)(D) f\right) \in L^{1}$, where $\mathfrak{R}_{j}$ denotes the $j$ th Riesz transform - that is, $\widehat{\mathfrak{R}_{j} f}(\xi):=-i \frac{\xi_{j}}{|\xi|} \widehat{f}(\xi)$, $j=1, \ldots, n$. We record here for future use the more familiar special case of Definition 2.2(iv) when $p=1$ :

$$
\|f\|_{h^{1}} \approx\|f\|_{L^{1}}+\sum_{j=1}^{n}\left\|\Re_{j}\left(\left(1-\psi_{0}\right)(D) f\right)\right\|_{L^{1}} \approx\left\|\psi_{0}(D) f\right\|_{L^{1}}+\left\|\left(1-\psi_{0}\right)(D) f\right\|_{H^{1}}
$$

The dual of the local Hardy space $h^{1}$ is the local BMO space, which is denoted bmo and consists of locally integrable functions that verify

$$
\|f\|_{\mathrm{bmo}}:=\|f\|_{\mathrm{BMO}}+\left\|\psi_{0}(D) f\right\|_{L^{\infty}}<\infty
$$

where BMO is the usual John-Nirenberg space of functions of bounded mean oscillation (see [24] for the definition) and $\psi_{0}$ is the cutoff function introduced in Definition 2.1. 
To bound the low-frequency part of an FIO, where the phase function is singular, we will make use of the following lemma, whose proof is a scholium of [6, Lemma 1.17], and therefore left to the reader:

Lemma 2.3. Let $a(\xi) \in C_{c}^{\infty}\left(\mathbb{R}^{n}\right)$ be supported in a neighbourhood of the origin. Assume also that $\varphi(\xi) \in C^{\infty}\left(\mathbb{R}^{n} \backslash 0\right)$ is positively homogeneous of degree 1 . Then for all $0 \leqslant \varepsilon<1$ we have

$$
\left|\int e^{i \varphi(\xi)-i x \cdot \xi} a(\xi) \mathrm{d} \xi\right| \lesssim\langle x\rangle^{-n-\varepsilon} .
$$

The following lemma will also prove useful in bounding the low-frequency part of an FIO. It is a consequence of a result due to Peetre [20].

Lemma 2.4. Let $f \in \mathfrak{C}^{1}\left(\mathbb{R}^{n}\right)$ have Fourier support contained inside the unit ball. Then for every $\rho>n$ and $r \in(n / \rho, 1]$, we have

$$
\left(\langle\cdot\rangle^{-\rho} *|f|\right)(x) \lesssim\left(M\left(|f|^{r}\right)(x)\right)^{1 / r}, \quad x \in \mathbb{R}^{n},
$$

where $M$ denotes the Hardy-Littlewood maximal function on $\mathbb{R}^{n}$.

Proof. As was shown by Peetre (see, for example, [26, Section 2.3.6]), for $r \geqslant n / \rho$ we have

$$
\sup _{y \in \mathbb{R}^{n}} \frac{|f(x-y)|}{\langle y\rangle^{\rho}} \lesssim\left(M\left(|f|^{r}\right)(x)\right)^{1 / r} .
$$

Now taking $r \in(n / \rho, 1]$ and using formula (13), we obtain

$$
\begin{aligned}
\left|\langle\cdot\rangle^{-\rho} * f(x)\right| & \lesssim \int_{\mathbb{R}^{n}} \frac{|f(x-y)|}{\langle y\rangle^{\rho}} \mathrm{d} y \leqslant\left(\sup _{y \in \mathbb{R}^{n}} \frac{|f(x-y)|}{\langle y\rangle^{\rho}}\right)^{1-r} \int_{\mathbb{R}^{n}} \frac{|f(x-y)|^{r}}{\langle y\rangle^{\rho r}} \mathrm{~d} y \\
& \lesssim\left(M\left(|f|^{r}\right)(x)\right)^{1 / r} .
\end{aligned}
$$

In the analysis of multilinear operators, a basic tool is a certain type of measure whose definition we now recall. A Borel measure $\mathrm{d} \mu(x, t)$ on $\mathbb{R}_{+}^{n+1}$ is called a Carleson measure if

$$
\|\mathrm{d} \mu\|_{\mathcal{e}}:=\sup _{Q} \frac{1}{|Q|} \int_{0}^{\ell(Q)} \int_{Q}|\mathrm{~d} \mu(x, t)|<\infty,
$$

where the supremum is taken over cubes all $Q \subset \mathbb{R}^{n}$ and $\ell(Q)$ denotes the diameter of $Q$ and $|Q|$ its Lebesgue measure. The quantity $\|\mathrm{d} \mu\|_{\mathcal{C}}$ is called the Carleson norm of $\mathrm{d} \mu$. In this paper we are exclusively interested in Carleson measures which are supported on lines parallel to the boundary of $\mathbb{R}_{+}^{n+1}$. More precisely, in what follows all Carleson measures will be supported on the set

$$
E:=\left\{(x, t): x \in \mathbb{R}^{n} \text { and } t=2^{-k} \text { for some } k \in \mathbb{Z}\right\},
$$

so they take the form

$$
\sum_{k \in \mathbb{Z}} \mathrm{d} \mu(x, t) \delta_{2^{-k}}(t)
$$

where $\delta_{2^{-k}}(t)$ is a Dirac measure at $2^{-k}$. This will be assumed throughout without further comment.

We recall some basic results concerning Carleson measures due to Carleson [4] which are also (as we shall see) useful in the context of multilinear operators. (See also Stein [24] for more streamlined and simplified proofs of the following results.) 
Lemma 2.5. If $\mathrm{d} \mu(x, t)$ is a Carleson measure, then

$$
\sum_{k} \int_{\mathbb{R}^{n}} F\left(x, 2^{-k}\right) \mathrm{d} \mu\left(x, 2^{-k}\right) \leqslant C_{n}\|\mathrm{~d} \mu\|_{\mathcal{e}} \int\left(\sup _{k} \sup _{|y-x|<2^{-k}}\left|F\left(y, 2^{-k}\right)\right|\right) \mathrm{d} x .
$$

Moreover, for $0<p<\infty$ we have

$$
\sum_{k} \int_{\mathbb{R}^{n}}\left|F\left(x, 2^{-k}\right)\right|^{p} \mathrm{~d} \mu\left(x, 2^{-k}\right) \leqslant C_{n}\|\mathrm{~d} \mu\|_{\mathcal{C}} \int\left(\sup _{k} \sup _{|y-x|<2^{-k}}\left|F\left(y, 2^{-k}\right)\right|\right)^{p} \mathrm{~d} x .
$$

Consequently, if $\varphi$ satisfies $|\varphi(x)| \lesssim\langle x\rangle^{-n-\varepsilon}$ (for some $0<\varepsilon<\infty$ ), then

$$
\sum_{k} \int_{\mathbb{R}^{n}}\left|\varphi\left(2^{-k} D\right) f(x)\right|^{2} \mathrm{~d} \mu\left(x, 2^{-k}\right) \leqslant C_{n}\|\mathrm{~d} \mu\|_{\mathcal{C}}\|f\|_{L^{2}}^{2},
$$

and if $\varphi$ is a bump function supported in a ball near the origin with $\phi(0)=1$, then we also have

$$
\sum_{k} \int_{\mathbb{R}^{n}}\left|\varphi\left(2^{-k} D\right) f(x)\right| \mathrm{d} \mu\left(x, 2^{-k}\right) \leqslant C_{n}\|\mathrm{~d} \mu\|_{\mathcal{C}}\|f\|_{h^{1}} .
$$

We also recall the quadratic estimate, which is a consequence of Plancherel's theorem: if $\varphi \in \mathcal{S}$ is such that $\varphi(0)=0$, then

$$
\sum_{k} \int\left|\varphi\left(2^{-k} D\right) f(x)\right|^{2} \mathrm{~d} x \lesssim\|f\|_{L^{2}}^{2}
$$

Finally, we shall also use the following result, which was stated and proved as [21, Lemma 4.10]:

Lemma 2.6. For any Carleson measure $\mathrm{d} \mu$ supported on $E$ and $K_{k}$ satisfying

$$
\left|K_{k}(x-y)\right| \lesssim 2^{k n}\left(1+\frac{|x-y|}{2^{-k}}\right)^{-n-\delta}
$$

for some $\delta>0$, we have

$$
\mathrm{d} \tilde{\mu}(x, t):=\sum_{k}\left(\int\left|K_{k}(x-y)\right| \mathrm{d} \mu(y, t)\right) \delta_{2^{-k}}(t) \mathrm{d} x,
$$

which defines a Carleson measure, and $\|\mathrm{d} \tilde{\mu}\|_{\mathrm{e}} \lesssim\|\mathrm{d} \mu\|_{\mathrm{e}}$.

As stated in Section 1, Theorem 1.4 is proved by interpolating between certain endpoint cases. In connection to those endpoint cases, the Hardy space $H^{1}$ and its dual BMO (see [24] for the definitions) will play an important role. In this context the following variant of [21, Corollary 4.12] will be useful:

Proposition 2.7. Let $\psi \in \mathcal{S}\left(\mathbb{R}^{n}\right)$ be supported in an annulus and $\phi \in \mathcal{S}\left(\mathbb{R}^{n}\right)$ satisfy $\phi(0)=0$. Then for any $F \in H^{1}, G \in \mathrm{BMO}$ and $v \in L_{k, x}^{\infty}$,

$$
\left|\int \sum_{k=-\infty}^{\infty} \psi\left(2^{-k} D\right) F(x) \phi\left(2^{-k} D\right) G(x) v\left(2^{-k}, x\right) \mathrm{d} x\right| \lesssim\|F\|_{H^{1}}\|G\|_{\mathrm{BMO}}\|v\|_{L_{k, x}^{\infty}} .
$$

\section{Global $h^{p} \rightarrow h^{p}$ boundedness of linear FIOs for $n /(n+1)<p \leqslant \infty$}

In this section we establish the global $h^{p}$ boundedness of a class of linear FIOs. This is formulated as Theorem 3.1 and will be needed to prove Theorem 1.4. Since $H^{1} \subset h^{1} \subset L^{1}$, this result strengthens 
the global $H^{1} \rightarrow L^{1}$ boundedness obtained by Ruzhansky and Sugimoto [22] for these FIOs. It also extends the local $h^{p} \rightarrow h^{p}$ boundedness of FIOs proven by Peloso and Secco [19] to a global result that is, we remove the requirement that the amplitude have compact $x$-support.

While this article was being written, this result was generalised further to cover more general phases and amplitudes, and Besov-Lipschitz as well as Triebel-Lizorkin spaces. This was carried out by Hassell, Portal and Rozendaal in [14] (in the realm of Hardy spaces), and by the first and third authors together with Israelsson in [15] (in the realm of Besov-Lipschitz and Triebel-Lizorkin spaces). Since the details of the following analysis are presented in [15] (albeit in a more involved setting), we concentrate here on presenting the main ideas of the $h^{p}$ boundedness of the FIOs that occur in this paper, and skip some of the technical details. The interested reader can find these details in [15].

Theorem 3.1. Set $m=-(n-1)\left|\frac{1}{p}-\frac{1}{2}\right|$ and $\frac{n}{n+1}<p \leqslant \infty$. Then any linear Fourier integral operator

$$
T_{\sigma}^{\varphi} f(x)=\int_{\mathbb{R}^{n}} \sigma(x, \xi) e^{i x \cdot \xi+i \varphi(\xi)} \widehat{f}(\xi) \mathrm{d} \xi,
$$

with an amplitude $\sigma(x, \xi) \in S^{m}$ and a phase function $\varphi$ (as in Definitions 1.1 and 1.3), satisfies the estimate

$$
\left\|T_{\sigma}^{\varphi} f\right\|_{X^{p}} \leqslant C\|f\|_{X^{p}}
$$

where $X^{p}$ is defined in definition (6).

We begin the proof of Theorem 3.1 by reducing to the case of $x$-independent amplitudes and $p<\infty$. We can write

$$
T_{\sigma}^{\varphi} f(x)=b(x, D) T_{\widetilde{\sigma}}^{\varphi} f(x),
$$

where $b(x, \xi)=\sigma(x, \xi)\langle\xi\rangle^{-m} \in S^{0}$ and $\widetilde{\sigma}=\langle\xi\rangle^{m} \in S^{m}$ is independent of $x$. Since pseudodifferential operators $b(x, D)$ are bounded on $X^{p}$ (see [11] for the case where $p \leqslant 1$ and $p=\infty$ and, for example, [24] for $1<p<\infty)$, to prove Theorem 3.1 we only need to prove the boundedness of $T_{\widetilde{\sigma}}^{\varphi}$. Since $T_{\widetilde{\sigma}}^{\varphi}$ is a self-adjoint operator, duality implies that the $p=\infty$ case follows immediately from the $p=1$ case. To avoid unnecessarily cumbersome notation, for the rest of the proof we drop the tilde and assume that $\sigma$ depends only on $\xi$.

Next we observe that the $L^{2}$ boundedness of $T_{\sigma}^{\varphi}$ is obvious when $\sigma$ does not depend on $x$, since it is a Fourier multiplier with bounded symbol (observe that $m \leqslant 0$ ). Therefore, we only need to consider $p \in(n /(n+1), 1]$, since once the theorem is proved for these values of $p$, the others follow by interpolation and duality.

We now split the operator into high- and low-frequency portions. Let $\chi(\xi)$ be a smooth cutoff function supported in the ball $B(0,1)$ and equal to 1 in $B(0,1 / 2)$. We set

$$
\sigma_{1}:=\chi(\xi) \sigma(\xi) \text { and } \quad \sigma_{2}(\xi):=(1-\chi(\xi)) \sigma(\xi)
$$

so $\sigma=\sigma_{1}+\sigma_{2}$. We shall study the boundedness of $T_{\sigma_{1}}^{\varphi}$ and $T_{\sigma_{2}}^{\varphi}$ separately, beginning with the estimates for $T_{\sigma_{1}}^{\varphi}$.

\subsection{Low-frequency analysis}

Our goal is to show that $T_{\sigma_{1}}^{\varphi}$ is bounded on $h^{p}$ for $\frac{n}{n+1}<p \leqslant 1$. For this we make use of Definition 2.2(ii) and let $\psi_{j}$ be a standard Littlewood-Paley partition of unity introduced in Definition 2.1.

Clearly the operator $\psi_{j}(D) T_{\sigma_{1}}^{\varphi}$ is an FIO with amplitude

$$
r_{j}(\xi)=\psi_{j}(\xi) \sigma_{1}(\xi)=\psi_{j}(\xi) \chi(\xi) \sigma(\xi)
$$


and phase function $x \cdot \xi+\varphi(\xi)$. The support properties of $\psi_{j}$ and $\chi$ imply that $r_{j}(\xi)=0$ for $j \geqslant 1$. This yields

$$
\left\|T_{\sigma_{1}}^{\varphi} f\right\|_{h^{p}}=\left\|\left(\sum_{j=0}^{\infty}\left|T_{r_{j}}^{\varphi} f\right|^{2}\right)^{\frac{1}{2}}\right\|_{L^{p}}=\left\|T_{r_{0}}^{\varphi} f\right\|_{L^{p}} .
$$

We can write

$$
T_{r_{0}}^{\varphi} f(x)=\int K(x, y)\left(\psi_{0}(D) f\right)(y) \mathrm{d} y,
$$

where $K(x, y)=\int \chi(\xi) \sigma(\xi) e^{i(x-y) \cdot \xi+i \varphi(\xi)} \mathrm{d} \xi$. By Lemma 2.3, we have $|K(x, y)| \lesssim\langle x-y\rangle^{-n-\varepsilon}$ for all $\varepsilon \in[0,1)$. Using this and Lemma 2.4 yields

$$
\left|T_{r_{0}}^{\varphi} f(x)\right| \lesssim\left|\left(\psi_{0}(D) f\right) *\langle\cdot\rangle^{-n-\varepsilon}\right| \lesssim\left(M\left(\left|\psi_{0}(D) f\right|^{r}\right)(x)\right)^{1 / r}
$$

for all $f \in \mathcal{S}, r \in\left(\frac{n}{n+\varepsilon}, 1\right)$ and $\varepsilon \in(0,1)$, where $M$ is the Hardy-Littlewood maximal function.

Thus, by choosing $\frac{n}{n+1}<r<p$ and making use of the boundedness of $M$ on $L^{p / r}$, we obtain

$$
\left\|T_{\sigma_{1}}^{\varphi} f\right\|_{h^{p}}=\left\|T_{r_{0}}^{\varphi} f\right\|_{L^{p}} \lesssim\left\|M\left(\left|\psi_{0}(D) f\right|^{r}\right)\right\|_{L^{p / r}}^{1 / r} \lesssim\left\|\psi_{0}(D) f\right\|_{L^{p}} \lesssim\|f\|_{h^{p}},
$$

where the last inequality follows by Definition 2.2(ii). A standard density argument yields the result.

\subsection{High-frequency analysis}

To analyse $T_{\sigma_{2}}^{\varphi}$ we need to use the atomic characterisation/decomposition of local Hardy spaces - that is, Definition 2.2(iii). It is also worth mentioning that the high-frequency case of the proof does not require the restriction $p \in(n / n+1,1]$, and works for all $p \in(0,1]$. Indeed, it is the lack of smoothness in the low-frequency part of the operator that leads to the counterexample in Section 4.

We also claim that in proving the boundedness of $T_{\sigma_{2}}^{\varphi}$ from $h^{p}$ to $h^{p}$, it is enough to show the boundedness of $T_{\sigma_{2}}^{\varphi}$ from $h^{p}$ to $L^{p}$. Assuming this claim, we first observe that the operators $r_{\varepsilon}^{\alpha}(D)$ and $\psi_{0}(D)$ in Definition 2.2(iv) are pseudodifferential operators with symbols in $S^{0}$ (uniformly in $\varepsilon$ ). As such, they map $h^{p}$ to itself by a result of Goldberg [11]. Now since $r_{\varepsilon}^{\alpha}(D) \circ T_{\sigma_{2}}^{\varphi}=T_{\sigma_{2}}^{\varphi} r_{\varepsilon}^{\alpha}(D)$ and $\psi_{0}(D) \circ T_{\sigma_{2}}^{\varphi}=T_{\sigma_{2}}^{\varphi} \psi_{0}(D)$, under the hypothesis of the claim both $r_{\varepsilon}^{\alpha}(D) \circ T_{\sigma_{2}}^{\varphi}$ and $\psi_{0}(D) \circ T_{\sigma_{2}}^{\varphi}$ map $h^{p}$ to $L^{p}$, and thus by Definition 2.2(iv) we would have that $T_{\sigma_{2}}^{\varphi}$ maps $h^{p}$ to itself. Therefore from now on our goal will be to prove the claim regarding the boundedness of $T_{\sigma_{2}}^{\varphi}$ from $h^{p}$ to $L^{p}$.

\subsubsection{Estimates of the norm on small balls}

Here we are concerned with estimates of the $L^{p}$-norm of $T_{\sigma_{2}}^{\varphi}$ for atoms $a$ supported in balls of radius smaller than 1 . To this end, we introduce a second frequency decomposition to the Littlewood-Paley decomposition of Definition 2.1. This was inspired by the work of Fefferman [7] and famously used by Seeger, Sogge and Stein in [23].

This section closely follows the same line of thought as [23], in which each Littlewood-Paley shell $\left\{\xi: 2^{j-1} \leqslant|\xi| \leqslant 2^{j+1}\right\}$ is further partitioned into $O\left(2^{j(n-1) / 2}\right)$ truncated cones of thickness $2^{j / 2}$. A clear exposition of the claims to follow can be found in [24, pp. 402-12].

For each $j \in \mathbb{N}$ we choose a collection of unit vectors $\left\{\xi_{j}^{v}\right\}_{v}$ such that

$\circ\left|\xi_{j}^{v}-\xi_{j}^{\nu^{\prime}}\right| \geqslant 2^{-\frac{j}{2}}$ for $v \neq v^{\prime}$ and

$\circ$ for each $\xi \in \mathbb{S}^{n-1}$, there exists a $\xi_{j}^{v}$ such that $\left|\xi-\xi_{j}^{v}\right|<2^{-j / 2}$, 
which is maximal with respect to the first property. It follows that it contains at most $O\left(2^{j(n-1) / 2}\right)$ elements. Associated to each $\xi_{j}^{v}$ is a cone

$$
\Gamma_{j}^{\nu}:=\left\{\xi \in \mathbb{R}^{n}:\left|\frac{\xi}{|\xi|}-\xi_{j}^{v}\right| \leqslant 2 \cdot 2^{-\frac{j}{2}}\right\}
$$

whose central axis lies along $\xi_{j}^{\nu}$.

One can construct a partition of unity

$$
\sum_{v} \chi_{j}^{v}=1
$$

of $\mathbb{R}^{n} \backslash\{0\}$ subordinate to $\left\{\Gamma_{j}^{v}\right\}_{j, v}$ which satisfies the estimates

$$
\left|\partial_{\xi}^{\alpha} \chi_{j}^{\nu}(\xi)\right| \leqslant C_{\alpha} 2^{j \frac{|\alpha|}{2}}|\xi|^{-|\alpha|}
$$

for all multi-indices $\alpha$, and the better estimate

$$
\left|\left(\xi_{j}^{v} \cdot \nabla\right)^{N} \chi_{j}^{v}(\xi)\right| \leqslant C_{N}|\xi|^{-N},
$$

for $N \geqslant 1$ along the direction $\xi_{j}^{v}$. Therefore, with $\psi_{j}$ from Definition 2.1,

$$
\psi_{0}(\xi)+\sum_{j=1}^{\infty} \sum_{v} \chi_{j}^{v}(\xi) \psi_{j}(\xi)=1, \quad \text { for all } \xi \in \mathbb{R}^{n}
$$

We now fix an $h^{p}$-atom $a$ supported in a ball $B(\bar{y}, r)$ with $r \leqslant 1$. We need to show that $\|T a\|_{L^{p}} \leqslant C$, where the constant $C$ does not depend on the atom $a$ or the radius of its support $r$. To do this we introduce the rectangles

$$
R_{j}^{v}=\left\{x \in \mathbb{R}^{n}:\left|x-\bar{y}+\nabla_{\xi} \varphi\left(\xi_{j}^{v}\right)\right| \leqslant A 2^{-\frac{j}{2}},\left|\pi_{j}^{v}\left(x-\bar{y}+\nabla_{\xi} \varphi\left(\xi_{j}^{v}\right)\right)\right| \leqslant A 2^{-j}\right\},
$$

where $\pi_{j}^{v}$ is the orthogonal projection in the direction $\xi_{j}^{v}$ and the size of the constant $A$ depends on the size of the Hessian $\partial_{\xi \xi}^{2} \varphi$ but not on $j$; and we define the 'region of influence' as

$$
B^{*}=\bigcup_{2^{-j} \leqslant r} \bigcup_{v} R_{j}^{v}
$$

We then split

$$
\int_{\mathbb{R}^{n}}\left|T_{\sigma_{2}}^{\varphi} a(x)\right|^{p} \mathrm{~d} x=\int_{B^{*}}\left|T_{\sigma_{2}}^{\varphi} a(x)\right|^{p} \mathrm{~d} x+\int_{B^{* c}}\left|T_{\sigma_{2}}^{\varphi} a(x)\right|^{p} \mathrm{~d} x .
$$

It can be shown that

$$
\left|B^{*}\right| \lesssim r
$$

so

$$
\int_{B^{*}}\left|T_{\sigma_{2}}^{\varphi} a(x)\right|^{p} \mathrm{~d} x \leqslant\left|B^{*}\right|^{1-p / 2}\left(\int_{B^{*}}\left|T_{\sigma_{2}}^{\varphi} a(x)\right|^{2} \mathrm{~d} x\right)^{p / 2} \lesssim r^{(1-p / 2)}\left\|T_{\sigma_{2}}^{\varphi} a\right\|_{L^{2}}^{p} .
$$

To estimate $\left\|T_{\sigma_{2}}^{\varphi} a\right\|_{L^{2}}^{p}$ we consider two cases: $-n / 2<m \leqslant 0$ and and $m \leqslant-n / 2$. 
In the case where $-n / 2<m \leqslant 0$, we can fix $q \in(1,2]$ which satisfies

$$
\frac{1}{2}=\frac{1}{q}+\frac{m}{n} \text {. }
$$

Using the $L^{2}$-boundedness of $T_{\sigma_{2}}^{\varphi} \circ\langle D\rangle^{-m}$ (which is clear when it is viewed as a 0th-order Fourier multiplier) and the $L^{q}$ to $L^{2}$ boundedness of the Riesz potential $\langle D\rangle^{m}$, we obtain

$$
\left\|T_{\sigma_{2}}^{\varphi} a\right\|_{L^{2}}^{p} \lesssim\|a\|_{L^{q}}^{p} \lesssim c|B|^{p / q-1} \lesssim r^{n(p / q-1)} .
$$

Combining this with formula (24), we obtain

$$
\int_{B^{*}}\left|T_{\sigma_{2}}^{\varphi} a(x)\right|^{p} \mathrm{~d} x \lesssim r^{(1-p / 2)+n(p / q-1)} \lesssim 1,
$$

where the last estimate follows from equation (25) because then

$$
\begin{aligned}
1-\frac{p}{2}+n\left(\frac{p}{q}-1\right) & =1-\frac{p}{2}+n\left(\frac{p}{2}-\frac{p m}{n}-1\right)=p\left(\frac{1}{p}-\frac{1}{2}+\frac{n}{2}-m-\frac{n}{p}\right) \\
& =p\left[-(n-1)\left(\frac{1}{p}-\frac{1}{2}\right)-m\right]=0 .
\end{aligned}
$$

If instead $m \leqslant \frac{-n}{2}$, then by setting $b=|B|^{1 / p-1 / q} a$, with $q$ once again satisfying equation (25) (so now $q<p<1$ ), we see that $b$ is an $h^{q}$-atom which is also supported in $B$. In fact, since $r \leqslant 1, b$ is even an atom in $H^{q}$, so by [16, Corollary 2.3], we have that $T_{\sigma_{2}}^{\varphi}$ is bounded from $H^{q}$ to $L^{2}$, and so

$$
\left.\int_{B^{*}}\right] a b s T_{\sigma_{2}}^{\varphi} a(x)^{p} \mathrm{~d} x \lesssim r^{(1-p / 2)}\|a\|_{H^{q}}^{p} \lesssim r^{(1-p / 2)}|B|^{(1 / q-1 / p) p}\|b\|_{H^{q}}^{p} \lesssim r^{(1-p / 2)+n(p / q-1)} \lesssim 1
$$

once again.

To analyse the second term on the right-hand side of equation (23) we use the partition of unity (22) and decompose

$$
T_{\sigma_{2}}^{\varphi}=\sum_{j=0}^{\infty} T_{j}=\sum_{j} \sum_{v} T_{j}^{v},
$$

where $T_{j}^{v}$ is the operator with kernel

$$
K_{j}^{v}(x, y)=\int \sigma(\xi) \chi_{j}^{v}(\xi) \psi_{j}(\xi) e^{i(x-y) \cdot \xi+i \varphi(\xi)} \mathrm{d} \xi .
$$

Since $\varphi$ is homogeneous of degree 1 , we can write $\varphi(\xi)=\nabla \varphi(\xi) \cdot \xi$ and so

$$
(x-y) \cdot \xi+\varphi(\xi)=\left(x-y+\nabla \varphi\left(\xi_{j}^{v}\right)\right) \cdot \xi+\left(\nabla \varphi(\xi)-\nabla \varphi\left(\xi_{j}^{v}\right)\right) \cdot \xi .
$$

Just as in [23], the kernel can therefore be written as

$$
K_{j}^{v}(x, y)=\int b_{j}^{\nu}(\xi) e^{i\left(x-y+\nabla \varphi\left(\xi_{j}^{\nu}\right)\right) \cdot \xi} \mathrm{d} \xi
$$

where $b_{j}^{\nu}(\xi):=\sigma(\xi) \chi_{j}^{\nu}(\xi) \psi_{j}(\xi) e^{i\left(\nabla \varphi(\xi)-\nabla \varphi\left(\xi_{j}^{\nu}\right)\right) \cdot \xi}$ satisfies the estimates

$$
\left|\partial^{\alpha} b_{j}^{\nu}(\xi)\right| \leqslant C_{\alpha} 2^{-j \frac{|\alpha|}{2}} \text { and }\left|\left(\xi_{j}^{v} \cdot \nabla\right)^{\mathscr{N}} b_{j}^{\nu}(\xi)\right| \leqslant C_{\mathscr{N}} 2^{-j \mathcal{N} / 2}
$$


for all multi-indices $\alpha$ and for $\mathcal{N} \geqslant 1$, in a similar way to formulas (20) and (21).

This leads to the kernel estimate

$$
\left|\partial_{y}^{\alpha} K_{j}^{v}(x, y)\right| \lesssim \frac{2^{j\left(m+\frac{n+1}{2}+|\alpha|\right)}}{\left(1+\left|2^{j} \pi_{j}^{\nu}\left(x-y+\nabla_{\xi} \varphi\left(\xi_{j}^{\nu}\right)\right)\right|^{2}\right)^{\mathcal{N}}\left(1+\left|2^{\frac{j}{2}}\left(x-y+\nabla_{\xi} \varphi\left(\xi_{j}^{\nu}\right)\right)^{\prime}\right|^{2}\right)^{\mathcal{N}}}
$$

for all multi-indices $\alpha$ and $\mathcal{N} \geqslant 0$, where $x^{\prime}$ denotes $x-\pi_{j}^{\nu}(x)$, the orthogonal complement to the projection in the direction $\xi_{j}^{v}$. (See [15, Lemma 3.2] for the details.)

To make use of this decomposition we estimate the second term on the right-hand side of equation (23) by

$$
\int_{B^{* c}}\left|T_{\sigma_{2}}^{\varphi} a(x)\right|^{p} \mathrm{~d} x \leqslant \sum_{2^{j}<r^{-1}} \int_{B^{* c}}\left|T_{j} a(x)\right|^{p} \mathrm{~d} x+\sum_{2^{j} \geqslant r^{-1}} \int_{B^{* c}}\left|T_{j} a(x)\right|^{p} \mathrm{~d} x .
$$

From formula (26) it is possible to prove that for $x \in B^{* c}$, any $\mathcal{N}$ and any $M \geqslant\left\lfloor n\left(\frac{1}{p}-1\right)_{+}\right\rfloor$, we have the pointwise estimates

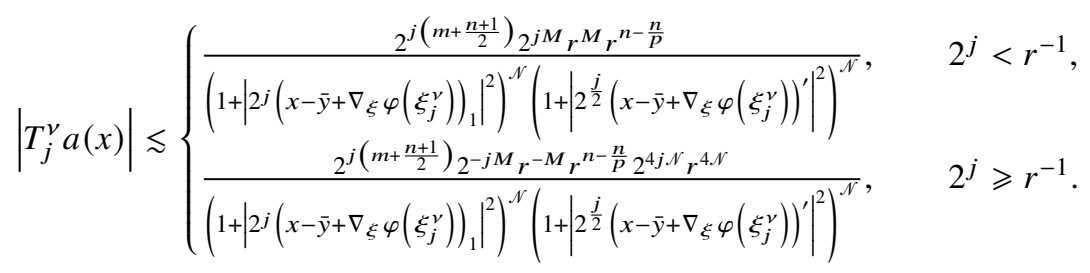

(See [15, Lemma 3.4] for details.) For the first term on the right-hand side of formula (27), we use the first estimate of formula (28) to deduce

$$
\int_{B^{* c}}\left|T_{j} a(x)\right|^{p} \mathrm{~d} x \lesssim 2^{j \frac{n-1}{2}} 2^{j p\left(m+\frac{n+1}{2}\right)} 2^{j M p} 2^{-j \frac{n+1}{2}} r^{M p} r^{n p-n} .
$$

(See [15, Proposition 6.2] for details.) Summing over $2^{j}<r^{-1}$ yields

$$
\sum_{2^{j}<r^{-1}} \int_{B^{* c}}\left|T_{j} a(x)\right|^{p} \mathrm{~d} x \lesssim 1
$$

if $M$ and $\mathcal{N}$ are chosen appropriately. For the second term in formula (27) we have that $2^{j} \geqslant r^{-1}$, and therefore using the second estimate of formula (28) yields

$$
\int_{B^{* c}}\left|T_{j} a(x)\right|^{p} \mathrm{~d} x \lesssim 2^{j \frac{n-1}{2}} 2^{j p\left(m+\frac{n+1}{2}\right)} 2^{-j M p} 2^{-j \frac{n+1}{2}} r^{-M p} r^{n p-n} 2^{4 j \mathcal{N} p} r^{4 \mathcal{N} p} .
$$

(See once again [15, Proposition 6.2] for details.) Summing over $2^{j} \geqslant r^{-1}$ yields

$$
\sum_{2^{j} \geqslant r^{-1}} \int_{B^{* c}}\left|T_{j} a(x)\right|^{p} \mathrm{~d} x \lesssim 1
$$

for appropriate $M$ and $\mathcal{N}$, which concludes the proof for atoms supported on balls of radius less than or equal to 1 .

\subsubsection{Estimates of the norm on large balls}

When the atom is supported on a ball with radius greater or equal to 1 , we use a strategy developed by Ruzhanksy and Sugimoto [22]. Once again, we wish to show $\left\|T_{\sigma_{2}}^{\varphi} a\right\|_{L^{p}} \lesssim 1$, where $a$ is an atom, but this 
time supported on a ball of radius $r \geqslant 1$. Without loss of generality, one can assume that this ball is centred at the origin. This is because the translation invariance of $L^{p}$ yields $\left\|T_{\sigma_{2}}^{\varphi} a\right\|_{L^{p}}=\left\|\tau_{s}^{*} T_{\sigma_{2}}^{\varphi} \tau_{s} \tau_{-s} a\right\|_{L^{p}}$, where $\tau_{s}$ is the operator of translation by $s \in \mathbb{R}^{n}$ and $\tau_{s}^{*} T_{\sigma_{2}}^{\varphi} \tau_{s}$ is exactly the same operator as $T_{\sigma_{2}}^{\varphi}$.

Following [22], we introduce the function

$$
H(z):=\inf _{\xi \in \mathbb{R}^{n}}|z+\nabla \varphi(\xi)|
$$

and its associated level sets

$$
\Delta_{r}:=\left\{z \in \mathbb{R}^{n} ; H(z) \geqslant r\right\}
$$

Clearly for $r_{1} \leqslant r_{2}$ we have $\Delta_{r_{1}} \supseteq \Delta_{r_{2}}$, and setting

$$
\begin{aligned}
\mathcal{M} & :=\sum_{|\gamma| \leqslant n+1} \sup _{x, y, \xi \in \mathbb{R}^{n}}\left|\partial_{\xi}^{\gamma} \sigma_{2}(\xi)\langle\xi\rangle^{-m_{c}(p)+|\gamma|}\right|, \\
\mathcal{N} & :=\sum_{1 \leqslant|\gamma| \leqslant n+2} \sup _{\xi \in \mathbb{R}^{n}}\left|\partial_{\xi}^{\gamma} \varphi(\xi)\langle\xi\rangle^{-1+|\gamma|}\right|,
\end{aligned}
$$

it is easy to check that both $\mathcal{M}$ and $\mathcal{N}$ are finite due to the decay, support and homogeneity properties of $\sigma$ and $\varphi$.

The following lemmas are special cases of [22, Theorem 2.2].

Lemma 3.2. Let $r \geqslant 1$. Then we have $\mathbb{R}^{n} \backslash \Delta_{2 r} \subseteq\{z:|z|<(2+\mathcal{N}) r\}$. Furthermore, for $x \in \Delta_{2 r}$ and $|y| \leqslant r$ we have

$$
H(x) \leqslant 2 H(x-y)
$$

and therefore $x-y \in \Delta_{r}$.

Lemma 3.3. The kernel

$$
K(z)=\int_{\mathbb{R}^{n}} \sigma_{2}(\xi) e^{i z \cdot \xi+i \varphi(\xi)} \mathrm{d} \xi
$$

of $T_{\sigma_{2}}^{\varphi}$ is smooth on $\bigcup_{r>0} \Delta_{r}$, and for all $L>n$ it satisfies

$$
\left\|H^{L} K\right\|_{L^{\infty}\left(\mathbb{R}^{n} \times \mathbb{R}^{n} \times \Delta_{r}\right)} \leqslant C(r, L, \mathcal{M}, \mathcal{N}),
$$

where $C(r, L, \mathcal{M}, \mathcal{N})$ is a positive constant depending only on $L, r>0, \mathcal{M}$ and $\mathcal{N}$. For $0<p \leqslant 1$ and $L>n / p$, the function $H(z)$ satisfies the bound

$$
\left\|H^{-L}\right\|_{L^{p}\left(\Delta_{r}\right)} \leqslant C(r, L, \mathcal{N}, p)
$$

Now returning to the problem of bounding the $L^{p}$-norm of $T_{\sigma_{2}}^{\varphi} a$, we split

$$
\left\|T_{\sigma_{2}}^{\varphi} a\right\|_{L^{p}\left(\mathbb{R}^{n}\right)} \leqslant\left\|T_{\sigma_{2}}^{\varphi} a\right\|_{L^{p}\left(\Delta_{2 r}\right)}+\left\|T_{\sigma_{2}}^{\varphi} a\right\|_{L^{p}\left(\mathbb{R}^{n} \backslash \Delta_{2 r}\right)} .
$$

We first estimate the integral in formula (30) over $\Delta_{2 r}$. For $x \in \Delta_{2 r}$ and $|y| \leqslant r$, Lemma 3.2 yields that $H(x) \leqslant 2 H(x-y)$ and $x-y \in \Delta_{r}$. This together with Lemma 3.3 in turn implies

$$
\begin{aligned}
\left|T_{\sigma_{2}}^{\varphi} a(x)\right| & \leqslant 2^{L} H(x)^{-L} \int_{|y| \leqslant r}\left|H(x-y)^{L} K(x-y) a(y)\right| \mathrm{d} y \\
& \leqslant 2^{L} H(x)^{-L}\left\|H^{L} K\right\|_{L^{\infty}\left(\mathbb{R}^{n} \times \Delta_{r}\right)}\|a\|_{L^{1}} \leqslant C(n, L, \mathcal{M}, \mathcal{N}) H(x)^{-L}
\end{aligned}
$$


for $x \in \Delta_{2 r}$, since $\|a\|_{L^{1}} \leqslant|B|^{1-1 / p}$ and $r \geqslant 1$. Therefore, choosing $L>n / p$, Lemma 3.3 and the monotonicity of $\Delta_{r}$ yield

$$
\left\|T_{\sigma_{2}}^{\varphi} a\right\|_{L^{p}\left(\Delta_{2 r}\right)} \leqslant\left\|H(x)^{-L}\right\|_{L^{p}\left(\Delta_{2 r}\right)} \leqslant C(n, \mathcal{M}, \mathcal{N}),
$$

as required.

For the integral in formula (30) over $\mathbb{R}^{n} \backslash \Delta_{2 r}$, Lemma 3.2 and Hölder's inequality yield

$$
\begin{aligned}
\left\|T_{\sigma_{2}}^{\varphi} a\right\|_{L^{p}\left(\mathbb{R}^{n} \backslash \Delta_{2 r}\right)} & \leqslant\left|\mathbb{R}^{n} \backslash \Delta_{2 r}\right|^{1-p / 2}\left\|T_{\sigma_{2}}^{\varphi} a\right\|_{L^{2}\left(\mathbb{R}^{n}\right)}^{p} \\
& \lesssim r^{n(1-p / 2)}\|a\|_{L^{2}\left(\mathbb{R}^{n}\right)}^{p} \lesssim 1,
\end{aligned}
$$

which together with formula (31) proves the estimate $\left\|T_{\sigma_{2}}^{\varphi} a\right\|_{L^{p}} \lesssim 1$.

\section{Examples showing the sharpness of results}

In Section 3.1 we only succeeded in proving that the low-frequency part of an FIO is bounded on $h^{p}$ for $p>n /(n+1)$. Here we shall constructively prove that the generic behaviour of an FIO acting on a Schwartz function is no better than $O\left(|x|^{-(n+1)}\right)$ as $|x| \rightarrow \infty$, and so we cannot expect the boundedness of an FIO into $h^{p} \subseteq L^{p}$ for $p \leqslant n /(n+1)$ to hold. More specifically, for each dimension $n$, we will find a function $f \in \mathcal{S} \subseteq h^{p}$ for which

$$
T(f)(x):=\int_{\mathbb{R}^{n}} \widehat{f}(\xi) e^{i x \cdot \xi+i|\xi|} \mathrm{d} \xi=\left(\frac{\Gamma\left(\frac{n+1}{2}\right) \widehat{f}(0)}{\pi^{(n+1) / 2} i}\right)|x|^{-(n+1)}+O\left(\frac{1+\log |x|}{|x|^{n+3}}\right)
$$

as $|x| \rightarrow \infty$. The function $f$ will be chosen so that $\widehat{f}$ has compact support, thus showing that regardless of the order of the decay of the amplitude, Theorem 3.1 cannot hold if $0<p \leqslant n /(n+1)$. In the case where $n=1$, this fact can also be proved directly, without the need for equation (33), using integration by parts. A different proof, again in the case where $n=1$, which yields the slightly stronger statement

$$
T(f)(x)=\left(\frac{\widehat{f}(0)}{\pi i}\right) \frac{1}{x^{2}}+O\left(x^{-4}\right)
$$

as $|x| \rightarrow \infty$ can be found in [15].

We consider here the case where $n>1$. For a function $f_{0}: \mathbb{R}^{+} \rightarrow \mathbb{C}$, we can define a radial function $f: \mathbb{R}^{n} \rightarrow \mathbb{C}$ by $f(x)=f_{0}(|x|)$ for all $x \in \mathbb{R}^{n}$. The Fourier transform of this $f$ is then also a radial function and can be used to define a transformation on $f_{0}$, as

$$
\mathcal{F}_{n}\left(f_{0}\right)(r):=\widehat{f}(\xi),
$$

where $r=|\xi|$. For $n>1$, the representation of the Fourier transform of a radial function (see, for example, [25, Chp 4, Thm 3.10]) together with properties of Bessel functions lead to the relation

$$
\mathcal{F}_{n}\left(f_{0}\right)=-\frac{1}{2 \pi} \mathcal{F}_{n+2}\left(f_{1}\right),
$$

for $f_{1}(r)=f_{0}^{\prime}(r) / r$, provided $f_{0}$ is continuously differentiable and

$$
f_{0}(r)= \begin{cases}O\left(r^{(1-n) / 2}\right) & \text { as } r \rightarrow \infty \\ O\left(r^{-n}\right) & \text { as } r \rightarrow 0 .\end{cases}
$$


In order to prove definition (32), choose $f$ to be a smooth radial function whose Fourier transform $\widehat{f}$ is compactly supported and equal to 1 in a neighbourhood of the origin. Furthermore, we set $g_{0}(r)=\widehat{f}(\xi)$ for $r=|\xi|$,

$$
\begin{aligned}
& g_{1}(r)=g_{0}(r) e^{i r}, \\
& g_{2}(r)=g_{0}(r)\left(e^{i r}-1-i r+r^{2} / 2\right), \\
& g_{3}(r)=g_{0}(r)\left(1-r^{2} / 2\right) \text { and } \\
& g_{4}(r)=g_{0}(r) i r .
\end{aligned}
$$

Then $T(f)(x)=(2 \pi)^{-n} \mathcal{F}_{n}\left(g_{1}\right)(|x|)$ and

$$
\mathcal{F}_{n}\left(g_{1}\right)=\mathcal{F}_{n}\left(g_{2}\right)+\mathcal{F}_{n}\left(g_{3}\right)+\mathcal{F}_{n}\left(g_{4}\right) .
$$

Since $x \mapsto g_{3}(|x|)$ is smooth and compactly supported, $\mathcal{F}_{n}\left(g_{3}\right)(r)=O\left(r^{-\mathcal{N}}\right)$ as $r \rightarrow \infty$ for each $\mathcal{N} \in \mathbb{N}$. We introduce a smooth cutoff function $\chi$ which is equal to 1 on the unit ball supported in the double of the unit ball. Thus,

$$
\begin{aligned}
\mathcal{F}_{n}\left(g_{2}\right)(|x|)= & \int_{\mathbb{R}^{n}} \widehat{f}(\xi)\left(e^{i|\xi|}-1-i|\xi|+\frac{|\xi|^{2}}{2}\right) e^{i x \cdot \xi} \mathrm{d} \xi \\
= & \int_{\mathbb{R}^{n}} \widehat{f}(\xi) \chi(\xi / \lambda)\left(e^{i|\xi|}-1-i|\xi|+\frac{|\xi|^{2}}{2}\right) e^{i x \cdot \xi} \mathrm{d} \xi \\
& +\int_{\mathbb{R}^{n}} \widehat{f}(\xi)(1-\chi(\xi / \lambda))\left(e^{i|\xi|}-1-i|\xi|+\frac{|\xi|^{2}}{2}\right) e^{i x \cdot \xi} \mathrm{d} \xi \\
= & A+B .
\end{aligned}
$$

To estimate $A$ and $B$ we can easily see that for $\xi \in \operatorname{supp}(\widehat{f})$ we have

$$
\left|\partial_{\xi}^{\alpha}\left(e^{i|\xi|}-1-i|\xi|+|\xi|^{2} / 2\right)\right| \lesssim|\xi|^{3-|\alpha|} .
$$

Therefore $A \lesssim \lambda^{n+3}$, and for each $\mathcal{N}$,

$$
\begin{array}{r}
|B|=\left|\int_{\mathbb{R}^{n}} \widehat{f}(\xi)(1-\chi(\xi / \lambda))\left(e^{i|\xi|}-1-i|\xi|+\frac{|\xi|^{2}}{2}\right)\left[\frac{x \cdot \nabla_{\xi}}{2 \pi i|x|^{2}}\right]^{\mathcal{N}}\left(e^{i x \cdot \xi}\right) \mathrm{d} \xi\right| \\
\lesssim \frac{1}{|x|^{\mathcal{N}}} \sum_{\left|\alpha_{1}\right|+\left|\alpha_{2}\right|+\left|\alpha_{3}\right|=\mathcal{N}} \int_{\mathbb{R}^{n}}\left|\partial^{\alpha_{1}} \widehat{f}(\xi)\right|\left|\partial^{\alpha_{2}}(1-\chi(\xi / \lambda))\right| \partial^{\alpha_{3}}\left(e^{i|\xi|}-1-i|\xi|+\frac{|\xi|^{2}}{2}\right) \mid \mathrm{d} \xi \\
\lesssim \frac{1}{|x|^{\mathcal{N}}} \sum_{\left|\alpha_{1}\right|+\left|\alpha_{2}\right|+\left|\alpha_{3}\right|=\mathcal{N},\left|\alpha_{2}\right|>0} \lambda^{-\left|\alpha_{2}\right|} \int_{|\xi| \sim \lambda}|\xi|^{3-\left|\alpha_{1}\right|-\left|\alpha_{3}\right|} \mathrm{d} \xi \\
+\frac{1}{|x|^{\mathcal{N}}} \sum_{\left|\alpha_{1}\right|+\left|\alpha_{3}\right|=\mathcal{N}} \int_{\lambda<|\xi| \lesssim 1}|\xi|^{-\left|\alpha_{1}\right|+3-\left|\alpha_{3}\right|} \mathrm{d} \xi
\end{array}
$$

where by splitting the sum we can take advantage of the different support properties of $(1-\chi(\xi / \lambda))$ and its derivatives. Taking $\mathcal{N}=n+3$, we find

$$
|B| \lesssim \frac{1}{|x|^{n+3}}(1-\log (\lambda)),
$$


and therefore taking $\lambda=1 /|x|$ yields

$$
\mathcal{F}_{n}\left(g_{2}\right)(|x|) \leqslant|A|+|B| \lesssim \frac{1}{|x|^{n+3}}(1+\log |x|),
$$

and thus $\mathcal{F}_{n}\left(g_{2}\right)(r)=O\left((1+\log r) / r^{n+3}\right)$.

To estimate $\mathcal{F}_{n}\left(g_{4}\right)$, we make use of equation (33). For this purpose, we define

$$
\begin{aligned}
& h_{0}(r)=g_{4}^{\prime}(r) / r, \\
& h_{1}(r)=i g_{0}^{\prime}(r), \\
& h_{2}(r)=i\left(g_{0}(r)-1\right) / r \quad \text { and } \\
& h_{3}(r)=i / r .
\end{aligned}
$$

Equation (33) then gives us

$$
\mathcal{F}_{n}\left(g_{4}\right)=-\frac{1}{2 \pi} \mathcal{F}_{n+2}\left(h_{0}\right)=-\frac{1}{2 \pi}\left(\mathcal{F}_{n+2}\left(h_{1}\right)+\mathcal{F}_{n+2}\left(h_{2}\right)+\mathcal{F}_{n+2}\left(h_{3}\right)\right) .
$$

We have that $\mathcal{F}_{n+2}\left(h_{1}\right)(r)=O\left(r^{-\mathcal{N}}\right)$ as $r \rightarrow \infty$ for each $\mathcal{N} \in \mathbb{N}$, since $h_{1}$ is smooth and compactly supported. It can also be shown that $\mathcal{F}_{n+2}\left(h_{2}\right)(r)=O\left(r^{-\mathcal{N}}\right)$ as $r \rightarrow \infty$ for each $\mathcal{N} \in \mathbb{N}$, since $h_{2}$ is smooth and its higher-order derivatives decay sufficiently rapidly. Moreover, $\mathcal{F}_{n+2}\left(h_{3}\right)(r)=$ $i 2^{n+1} \pi^{(n+1) / 2} \Gamma\left(\frac{n+1}{2}\right) r^{-(n+1)}$ (as can be found in, for example, [25, Chp 4, Thm 4.1]). Putting these together, we find that

$$
\mathcal{F}_{n}\left(g_{4}\right)=-\left(2^{n} \pi^{(n-1) / 2} \Gamma\left(\frac{n+1}{2}\right) i\right) r^{-(n+1)}+O\left(r^{-\mathcal{N}}\right)
$$

as $r \rightarrow \infty$ for each $\mathcal{N} \in \mathbb{N}$, and therefore we have proved definition (32) in the case where $n>1$.

Before we turn to the proof of Theorem 1.4, we provide an example to show that the value of $m$ in formula (8) is sharp. We do this in the case where $N=2, p_{0}=2$ and $p_{1}=p_{2}=4$. Consider the bilinear operator

$$
U(f, g)(x)=\int_{\mathbb{R}^{2 n}} a(\xi, \eta) \widehat{f}(\xi) \widehat{g}(\eta) e^{i x \cdot(\xi+\eta)} e^{i|\xi|-i|\eta|+i|\xi+\eta|} \mathrm{d} \xi \mathrm{d} \eta
$$

where

$$
a(\xi, \eta)=\sum_{k=1}^{\infty} \psi_{j}(\xi) \overline{\psi_{j}(-\eta)}|\xi|^{m / 2}|\eta|^{m / 2}
$$

the $\psi_{j}$ are a Littlewood-Paley partition of unity as defined in Definition 2.1 and $m$ fails to satisfy formula (8), so

$$
m=-(n-1)\left(\left|\frac{1}{p_{1}}-\frac{1}{2}\right|+\left|\frac{1}{p_{2}}-\frac{1}{2}\right|+\left|\frac{1}{p_{0}}-\frac{1}{2}\right|\right)+\varepsilon=-\frac{(n-1)}{2}+\varepsilon
$$

for $\varepsilon>0$. We can write $U(f, g)=e^{i \sqrt{-\Delta}}(T(f, g))$ for

$$
T(f, g)(x)=\int_{\mathbb{R}^{2 n}} a(\xi, \eta) \widehat{f}(\xi) \widehat{g}(\eta) e^{i x \cdot(\xi+\eta)} e^{i|\xi|-i|\eta|} \mathrm{d} \xi \mathrm{d} \eta,
$$


so the unitarity of $e^{i \sqrt{-\Delta}}$ implies that the boundedness of $U$ from $L^{4} \times L^{4}$ to $L^{2}$ is equivalent to the boundedness of $T$ on the same spaces. We compute

$$
\begin{aligned}
T(f, \bar{f})(x) & =\int_{\mathbb{R}^{2 n}}\left(\sum_{k=1}^{\infty} \psi_{j}(\xi) \psi_{j}(-\eta)|\xi|^{m / 2}|\eta|^{m / 2}\right) \widehat{f}(\xi) \widehat{f}(-\eta) e^{i x \cdot(\xi+\eta)} e^{i|\xi|-i|\eta|} \mathrm{d} \xi \mathrm{d} \eta \\
& =\sum_{k=1}^{\infty}\left(\int_{\mathbb{R}^{n}} \psi_{j}(\xi)|\xi|^{m / 2} \widehat{f}(\xi) e^{i x \cdot \xi} e^{i|\xi|} \mathrm{d} \xi\right)\left(\int_{\mathbb{R}^{n}} \overline{\psi_{j}(-\eta)}|\eta|^{m / 2} \overline{\widehat{f}(-\eta)} e^{i x \cdot \eta} e^{-i|\eta|} \mathrm{d} \eta\right) \\
& =\sum_{k=1}^{\infty}\left(\int_{\mathbb{R}^{n}} \psi_{j}(\xi)|\xi|^{m / 2} \widehat{f}(\xi) e^{i x \cdot \xi} e^{i|\xi|} \mathrm{d} \xi\right) \overline{\left(\int_{\mathbb{R}^{n}} \psi_{j}(\xi)|\xi|^{m / 2} \widehat{f}(\xi) e^{i x \cdot \xi} e^{i|\xi|} \mathrm{d} \xi\right)} \\
& =\left.\left.\sum_{k=1}^{\infty}\left|\int_{\mathbb{R}^{n}} \psi_{j}(\xi)\right| \xi\right|^{m / 2} \widehat{f}(\xi) e^{i x \cdot \xi} e^{i|\xi|} \mathrm{d} \xi\right|^{2}=\sum_{k=1}^{\infty}\left|\psi_{j}(D)(S(f))(x)\right|^{2},
\end{aligned}
$$

where

$$
S(f)(x)=\int_{\mathbb{R}^{n}}\left(1-\psi_{0}(\xi)\right)|\xi|^{m / 2} \widehat{f}(\xi) e^{i x \cdot \xi} e^{i|\xi|} \mathrm{d} \xi
$$

If we assume that $T$ is bounded from $L^{4} \times L^{4}$ to $L^{2}$ and $f \in L^{4}$, then [12, Theorem 6.1.2, estimate (6.1.8)] says that there exists a polynomial $Q$ such that we can estimate

$$
\begin{aligned}
\|S(f)-Q\|_{L^{4}} & \lesssim\left\|\left(\sum_{k=1}^{\infty}\left|\psi_{j}(D)(S(f))\right|^{2}\right)^{1 / 2}\right\|_{L^{4}} \\
& =\left\|\sum_{k=1}^{\infty}\left|\psi_{j}(D)(S(f))\right|^{2}\right\|_{L^{2}}^{1 / 2}=\|T(f, \bar{f})\|_{L^{2}}^{1 / 2} \lesssim\|f\|_{L^{4}} .
\end{aligned}
$$

However, if we let $\widehat{f}(\xi)=\left(1-\psi_{0}(\xi)\right)|\xi|^{-\lambda} e^{-i|\xi|}$ with

$$
\frac{2 n+1}{4}<\lambda<\frac{2 n+1}{4}+\frac{\varepsilon}{2},
$$

then [18, p. 302, fact (II-i)] shows us that $f \in L^{4}$ but $S(f) \notin L^{4}$. Moreover, $S(f)(x) \sim|x|^{-(n+\varepsilon) / 4}$ as $|x| \rightarrow 0$, so regardless which polynomial $Q$ is, estimate (34) leads to a contradiction. Therefore neither $T$ nor $U$ can be bounded operators from $L^{4} \times L^{4}$ to $L^{2}$.

\section{The identification of the endpoint cases}

In order to prove Theorem 1.4, we wish to identify the various values of the exponents $p_{1}, p_{2}, \ldots, p_{N}$ from which the general result claimed in Theorem 1.4 will follow via interpolation. These specific values are called endpoint cases, and to identify them we define the continuous convex piecewise linear function

$$
F(x)=\left\|x-\frac{1}{2} \mathbf{1}\right\|_{\ell^{1}}+\left|x \cdot \mathbf{1}-\frac{1}{2}\right|, \quad \text { for } x \in \mathbb{R}^{N},
$$

where $\mathbf{1}=(1, \ldots, 1) \in \mathbb{R}^{N}$. Bearing in mind that $p_{0}$ satisfies equation (7), the right-hand side of formula (8) can be written as

$$
-(n-1) F\left(1 / p_{1}, \ldots, 1 / p_{N}\right) .
$$


The fact that we are restricting our attention to exponents $1 \leqslant p_{j} \leqslant \infty(j=0,1, \ldots, N)$ means we are interested in the behaviour of $F$ on the domain

$$
D:=\left\{x \in[0,1]^{N}: x \cdot \mathbf{1} \leqslant 1\right\}
$$

and in understanding the set

$$
\{(x, s) \in D \times[0, \infty): F(x) \leqslant s\} .
$$

Since $F$ is convex and piecewise linear, this set is a convex unbounded polytope. Its extreme points lie on the graph of $F$ over $D$ and are in one-to-one correspondence with the extreme points of the subsets of $D$ on which $F$ is linear. The subsets of $D$ on which $F$ is linear, as intersections of the compact convex set $D$ with convex sets (in this case half-spaces), are compact and convex. By the Krein-Milman theorem, these subsets of $D$ are the closed convex hull of their extreme points. Thus our task is to identify these convex sets and their extreme points. This is the content of the following theorem:

Theorem 5.1. If $\left\{e_{j}\right\}_{j}$ is the standard basis in $\mathbb{R}^{N}$, then the set $D$ defined in definition (36) can be written as the union

$$
D=D_{0}^{0} \cup D_{0}^{1} \cup\left(\cup_{j=1}^{N} D_{1}^{j}\right)
$$

where

$$
\begin{aligned}
& D_{0}^{0}=\operatorname{Hull}\left(\{0\} \cup\left\{\frac{e_{k}}{2}\right\}_{k=1}^{N}\right) \\
& D_{0}^{1}=\operatorname{Hull}\left(\left\{\frac{e_{k}}{2}\right\}_{k=1}^{N} \cup\left\{\frac{e_{k}+e_{\ell}}{2}\right\}_{k, \ell=1}^{N}\right) \\
& D_{1}^{j}=\operatorname{Hull}\left(\left\{e_{j}, \frac{e_{j}}{2}\right\} \cup\left\{\frac{e_{j}+e_{k}}{2}\right\}_{k \neq j}\right) .
\end{aligned}
$$

Moreover, $F$ defined in equation (35) is a linear function on each of these sets.

Before proving Theorem 5.1, we observe that the values $\left(1 / p_{1}, \ldots, 1 / p_{N}\right)$ corresponding to the endpoint cases we need to consider are exactly the points of the set

$$
\{0\} \cup\left\{\frac{e_{k}}{2}\right\}_{k=1}^{N} \cup\left\{\frac{e_{k}+e_{\ell}}{2}\right\}_{k, \ell=1}^{N} \cup\left\{e_{k}\right\}_{k=1}^{N} .
$$

This leads to the following corollary:

Corollary 5.2. It is sufficient to prove Theorem 1.4 for the following values of exponents:

(i) $p_{j}=\infty$ for all $j=0, \ldots N$;

(ii) $p_{0}=2$; and for any $1 \leqslant j \leqslant N, p_{j}=2$; and $p_{k}=\infty$ for $k \neq j$;

(iii) $p_{0}=1$; and for any pair $1 \leqslant j_{1}<j_{2} \leqslant N, p_{j_{1}}=p_{j_{2}}=2$; and $p_{k}=\infty$ for $j_{1} \neq k \neq j_{2}$; and

(iv) $p_{0}=1$; and for any $1 \leqslant j \leqslant N, p_{j}=1$; and $p_{k}=\infty$ for $k \neq j$.

Proof. The proof is a fairly standard application of multilinear interpolation theory as described in [13], using known results for interpolation spaces (for example, [17, Theorem 11]. Therefore we confine ourselves to a brief sketch. We define an analytic family of operators $\left\{T_{z}\right\}$ on the strip $0<\operatorname{Re}(z) \leqslant 1$, where $T_{z}$ is given by equation (4) with an amplitude $\sigma_{z}(x, \Xi)$, where $\sigma_{z}(x, \Xi)=\sigma(x, \Xi)\left(1+\sum_{j=1}^{N}\left|\xi_{j}\right|^{2}\right)^{\frac{\gamma(z)}{2}}$ and $\gamma(z)=-m-z Q$, with $Q$ chosen so that when $\operatorname{Re}(z)=0$ and $\operatorname{Re}(z)=1$, we have $\sigma_{z} \in S^{\alpha}(n, N)$ and $\sigma_{z} \in S^{\beta}(n, N)$, where $\alpha$ and $\beta$ correspond to two different extremal cases as given in the statement of the corollary. Noting that in both cases the seminorms of $\sigma_{z}$ depend polynomially on $|\operatorname{Im} z|$, the validity of the bounds for the extremal cases together with the aforementioned result of Grafakos and Mastyło [13, Theorem 1.1] yield the desired results. 
Proof of Theorem 5.1. Let $N^{\prime}=N^{\prime}(x)$ denote the number of coordinates such that $x_{j} \geqslant 1 / 2$ (for $j=1, \ldots, N)$. That $x \in D$ means $\sum_{j=1}^{N} x_{j} \leqslant 1$, which in turn implies that $N^{\prime} \in\{0,1,2\}$. We can therefore decompose $D=D_{0} \cup D_{1} \cup D_{2}$, where for each $k=0,1,2$,

$$
D_{k} \text { is the closure of the set of points } x \in D \text { for which } N^{\prime}(x)=k \text {. }
$$

We observe that $D_{2}$ consists exactly of the vertices $\frac{1}{2}\left(e_{j}+e_{k}\right)$ for $1 \leqslant j<k \leqslant N$, and it is easy to check that these points are limit points of $D_{1}$. Therefore $D_{2} \subset D_{1}$ and

$$
D=D_{0} \cup D_{1}
$$

We can further decompose

$$
D_{0}=D_{0}^{0} \cup D_{0}^{1}
$$

where

$$
D_{0}^{0}=\left\{x \in D_{0}: 0 \leqslant x \cdot \mathbf{1} \leqslant \frac{1}{2}\right\} \quad \text { and } \quad D_{0}^{1}=\left\{x \in D_{0}: \frac{1}{2} \leqslant x \cdot \mathbf{1} \leqslant 1\right\}
$$

Since $0 \leqslant x \cdot \mathbf{1}<\frac{1}{2}$ and $x_{j} \geqslant 0$ for $x=\left(x_{1}, \ldots, x_{N}\right) \in D_{0}^{0}$, all points $x \in D_{0}^{0}$ can be expressed as the convex hull of the points 0 , and $\frac{1}{2} e_{k}$, for $k=1, \ldots, N$. Therefore, $D_{0}^{0}=\operatorname{Hull}\left(\left\{0, \frac{e_{1}}{2}, \ldots, \frac{e_{N}}{2}\right\}\right)$.

Leaving $D_{0}^{1}$ for a moment, we next consider $D_{1}$. We can write

$$
D_{1}=\cup_{j=1}^{n} D_{1}^{j},
$$

where

$$
D_{1}^{j}=\left\{x \in D_{1}: x_{j} \geqslant \frac{1}{2} \geqslant x_{k} \text { for all } k \neq j\right\}
$$

Note that $D_{1}^{j}$ is the translation of $D_{0}^{0}$ by $\frac{e_{j}}{2}$, so it follows that

$$
\begin{aligned}
D_{1}^{j} & =\frac{e_{j}}{2}+D_{0}^{0} \\
& =\frac{e_{j}}{2}+\operatorname{Hull}\left(\left\{0, \frac{e_{1}}{2}, \ldots, \frac{e_{N}}{2}\right\}\right) \\
& =\operatorname{Hull}\left(\left\{e_{j}, \frac{e_{j}}{2}\right\} \cup\left\{\frac{e_{j}+e_{k}}{2}\right\}_{k \neq j}\right) .
\end{aligned}
$$

We now return to $D_{0}^{1}$. Given a fixed arbitrary point $x \in D_{0}^{1}$, consider the maximal line segment contained in the ray from the origin through $x$ which is contained in $D_{0}^{1}$. This is a set of the form

$$
\left\{y=\lambda x: \lambda_{-} \leqslant \lambda \leqslant \lambda_{+}\right\}
$$

The factor $\lambda_{-}$will be determined by when the ray breaks through the plane $y \cdot \mathbf{1}=1 / 2$, so $\lambda_{-}$solves the equation $\lambda_{-} x \cdot \mathbf{1}=1 / 2$, and $\lambda_{+}$will be determined by when the ray first breaks through one of the planes $y \cdot e_{j}=1 / 2(j=1, \ldots, N)$ and $y \cdot \mathbf{1}=1$; therefore,

$$
\lambda_{+}=\min \left\{\lambda_{j}: \lambda_{j} x \cdot e_{j}=1 / 2(j=1, \ldots, N) \text { and } \lambda_{N+1} x \cdot \mathbf{1}=1\right\} .
$$



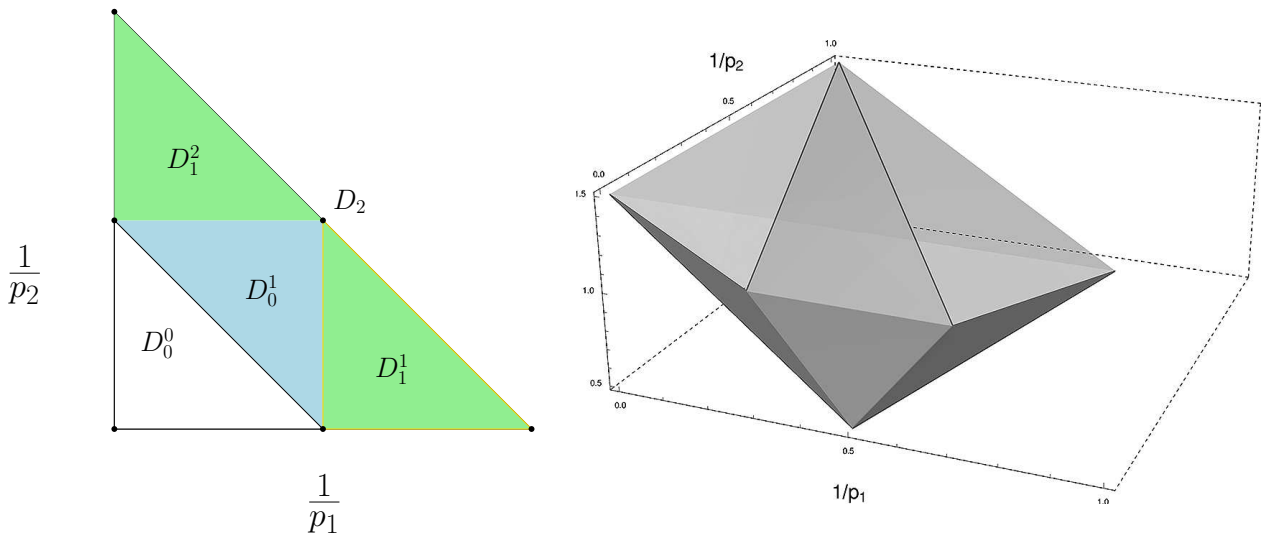

Figure 1. Decomposition of $D$ and graph of $F$ for $N=2$.

However,

$$
\begin{gathered}
\lambda_{-} x \in \operatorname{Hull}\left(\left\{\frac{e_{1}}{2}, \ldots, \frac{e_{N}}{2}\right\}\right), \\
\lambda_{j} x \in \operatorname{Hull}\left(\left\{\frac{e_{j}}{2}\right\} \cup\left\{\frac{e_{j}+e_{k}}{2}\right\}_{k \neq j}\right) \quad \text { if } \lambda_{+}=\lambda_{j} \text { for } j=1, \ldots, N \text { and } \\
\lambda_{N+1} x \in \operatorname{Hull}\left(\left\{\frac{e_{k}+e_{\ell}}{2}\right\}_{k \neq \ell}\right) \quad \text { if } \lambda_{+}=\lambda_{N+1},
\end{gathered}
$$

so it follows that

$$
x \in \operatorname{Hull}\left(\left\{\frac{e_{k}}{2}\right\}_{k=1}^{N} \cup\left\{\frac{e_{k}+e_{\ell}}{2}\right\}_{k, \ell=1}^{N}\right) .
$$

Summarising, we can write

$$
D=D_{0}^{0} \cup D_{0}^{1} \cup\left(\cup_{j=1}^{N} D_{1}^{j}\right),
$$

where each set is convex and the extreme points are the ones given in the statement of Theorem 5.1 (See Figure 1).

We now check that $F$ is linear on these sets. For $x \in D_{0}, x_{k} \leqslant \frac{1}{2}$ for all $k$, so we have

$$
\begin{aligned}
F(x) & =\sum_{k=1}^{N}\left(\frac{1}{2}-x_{k}\right)+\left|\sum_{k=1}^{N} x_{k}-\frac{1}{2}\right| \\
& = \begin{cases}\frac{N-1}{2} & \text { if } x \in D_{0}^{1}, \\
\frac{N-1}{2}+(1-2 x \cdot \mathbf{1}) & \text { if } x \in D_{0}^{0} .\end{cases}
\end{aligned}
$$

Now if $x \in D_{1}^{j}$, then $x_{k} \leqslant \frac{1}{2} \leqslant x_{j}$ for all $k \neq j$, and so $x \cdot \mathbf{1} \geqslant 1 / 2$. Thus we can write

$$
\begin{aligned}
F(x) & =\sum_{k \neq j}\left(\frac{1}{2}-x_{k}\right)+\left(x_{j}-\frac{1}{2}\right)+\sum_{k=1}^{N} x_{k}-\frac{1}{2} \\
& =\frac{N-1}{2}+2 x_{j}-1 .
\end{aligned}
$$

Thus, in all cases, we see that $F$ is linear on each of the convex sets $D_{0}^{0}, D_{0}^{1}$ and $D_{1}^{j}$, for $j=$ $1, \ldots, N$. 


\section{Frequency decomposition of multilinear FIOs}

In what follows we shall demonstrate that the regularity of $T_{\sigma}^{\Phi}$ can be obtained by considering three frequency regimes: when $\Xi$ lies inside a compact set, when one component of $\Xi=\left(\xi_{1}, \ldots, \xi_{N}\right)$ dominates the other; and when two fixed components of $\left(\xi_{1}, \ldots, \xi_{N}\right)$ are comparable to each other.

In all that follows we take $N>1$. First we define the component of $\sigma$ with frequency support contained in a compact set. We introduce the cutoff function $\chi: \mathbb{R}^{n N} \rightarrow \mathbb{R}$, such that $\chi(\Xi)=1$ for $|\Xi| \leqslant 1 / 8$ and $\chi(\Xi)=0$ for $|\Xi| \geqslant 1 / 4$, and define

$$
\sigma_{0}(x, \Xi)=\chi(\Xi) \sigma(x, \Xi) .
$$

To define the components of $\sigma$ where one frequency dominates all the others, we construct a cutoff function $v: \mathbb{R}^{n N} \rightarrow \mathbb{R}$ such that $v(\Xi)=0$ for $\left|\xi_{1}\right| \leqslant 32 \sqrt{N-1}\left|\Xi^{\prime}\right|$ and $v(\Xi)=1$ for $64 \sqrt{N-1}\left|\Xi^{\prime}\right| \leqslant$ $\left|\xi_{1}\right|$, where $\Xi^{\prime}:=\left(\xi_{2}, \ldots, \xi_{N}\right)$. This can be done by taking $\lambda \in \mathcal{C}^{\infty}(\mathbb{R})$ such that $\lambda(t)=1$ if $t \leqslant c_{1}$ and $\lambda(t)=0$ if $t \geqslant c_{2}$ for two real numbers $0<c_{1}<c_{2}<1$, which will be decided momentarily. Define

$$
v(\Xi)=1-\lambda\left(\frac{\left|\xi_{1}\right|^{2}}{|\Xi|^{2}}\right) \in \mathcal{C}^{\infty}\left(\mathbb{R}^{n N} \backslash 0\right)
$$

By construction, it follows that

$$
v(\Xi)=\left\{\begin{array}{ll}
0 & \text { if }\left|\xi_{1}\right|^{2} \leqslant c_{1}|\Xi|^{2} \\
1 & \text { if }\left|\xi_{1}\right|^{2} \geqslant c_{2}|\Xi|^{2}
\end{array},= \begin{cases}0 & \text { if }\left|\xi_{1}\right| \leqslant \sqrt{\frac{c_{1}}{1-c_{1}}}\left|\Xi^{\prime}\right|, \\
1 & \text { if }\left|\xi_{1}\right| \geqslant \sqrt{\frac{c_{2}}{1-c_{2}}}\left|\Xi^{\prime}\right|,\end{cases}\right.
$$

and a calculation shows that taking

$$
\begin{aligned}
& c_{1}=1-\frac{1}{1+32^{2}(N-1)}=\frac{2^{10}(N-1)}{1+2^{10}(N-1)}, \\
& c_{2}=1-\frac{1}{1+4 \cdot 32^{2}(N-1)}=\frac{2^{12}(N-1)}{1+2^{12}(N-1)},
\end{aligned}
$$

ensures that we obtain the function $v$ with the required properties. Given $j=1, \ldots N$, we define $\Xi_{j}^{\prime}:=\left(\xi_{1}, \ldots, \xi_{j-1}, \xi_{j+1}, \ldots, \xi_{N}\right)$ and

$$
v_{j}(\Xi):=v\left(\xi_{j}, \Xi_{j}^{\prime}\right)
$$

for all $\Xi \in \mathbb{R}^{n N}$. We then define the component of $\sigma$ for which $\xi_{j}$ dominates the other frequency components to be

$$
\sigma_{j}(x, \Xi)=(1-\chi(\Xi)) v_{j}(\Xi) \sigma(x, \Xi), \quad \text { for } j=1, \ldots N
$$

What remains of $\sigma$ will be split into functions on whose support two frequency components are comparable. Observe that the supports of the $v_{j}$ are disjoint, and therefore the $\Xi$-support of

$$
\sigma(x, \Xi)-\sum_{j=0}^{N} \sigma_{j}(x, \Xi)
$$

is contained in the set of all $\Xi$ for which no $v_{j}(\Xi)$ is equal to 1 . We define

$$
\widetilde{v}(\Xi)=1-\lambda\left(\frac{\left|\xi_{1}\right|^{2}}{c_{3}|\Xi|^{2}}\right) \in \mathcal{C}^{\infty}\left(\mathbb{R}^{n N} \backslash 0\right)
$$


for some constant $0<c_{3}<1$ (to be chosen momentarily) and

$$
\widetilde{v}_{j}(\Xi):=\widetilde{v}\left(\xi_{j}, \Xi_{j}^{\prime}\right)
$$

For fixed $k$, if $\Xi$ is not contained in the support of $\widetilde{v}_{j}$ for any $j \neq k$, then $\left|\xi_{j}\right|^{2} \leqslant c_{3} c_{1}|\Xi|^{2}$ for all $j \neq k$, and consequently

$$
\left|\xi_{k}\right|^{2} \geqslant\left(1-c_{3} c_{1}(N-1)\right)|\Xi|^{2}
$$

Thus, we choose $c_{3}$ so that $1-c_{3} c_{1}(N-1)>c_{2}$, and all $\Xi$ which are not contained in the support of $\widetilde{v}_{j}$ for any $j \neq k$ will be such that $v_{k}(\Xi)=1$. Therefore the functions

$$
\Theta_{j, k}(\Xi):=\frac{\widetilde{v}_{j}(\Xi) \widetilde{v}_{k}(\Xi)}{\left(\sum_{\ell=1}^{N} \widetilde{v}_{\ell}(\Xi)\right)^{2}}
$$

are a smooth partition of the $\Xi$-support of expression (38), and $\left|\xi_{j}\right| \approx\left|\xi_{k}\right|$ on the support of $\Theta_{j, k}$. Defining

$$
\sigma_{j, k}(x, \Xi)=(1-\chi(\Xi)) \Theta_{j, k}(\Xi)\left(\sigma(x, \Xi)-\sum_{\ell=0}^{N} \sigma_{\ell}(x, \Xi)\right), \quad \text { for } j, k=1, \ldots N,
$$

we have completed our decomposition of the amplitude $\sigma$ as

$$
\sigma(x, \Xi)=\sigma_{0}(x, \Xi)+\sum_{j=1}^{N} \sigma_{j}(x, \Xi)+\sum_{j \neq k} \sigma_{j, k}(x, \Xi),
$$

where $\sigma_{0}$ has compact $\Xi$-support, $\left|\xi_{j}\right|$ dominates $|\Xi|$ on the $\Xi$-support of $\sigma_{j}$ and $\left|\xi_{j}\right| \approx\left|\xi_{k}\right|$ on the $\Xi$-support of $\sigma_{j, k}$.

It is easy to check that if $\sigma \in S^{m}(n, N)$, then $\sigma_{j}$ and $\sigma_{j, k}$ are also in $S^{m}(n, N)$ for all $j, k=1, \ldots, N$, and $\sigma_{0} \in S^{\mu}(n, N)$ for all $\mu \in \mathbb{R}$.

\section{Boundedness results for $T_{\sigma_{j}}^{\Phi}$}

We will restrict our discussion to the amplitude $\sigma_{1}$. This will be sufficient for the treatment of an arbitrary $\sigma_{j}$, since a permutation of the frequency variables $\xi_{1}, \ldots, \xi_{N}$ reduces the boundedness of $\sigma_{j}$ in one of the endpoint cases from Corollary 5.2 to an endpoint case for $\sigma_{1}$.

We begin by decomposing $\sigma_{1}$ in a similar fashion to Coifman and Meyer [5]. The rough idea is to first introduce a Littlewood-Paley partition of unity in the $\xi_{1}$ variable. We can then make use of the fact that $|\Xi| \lesssim\left|\xi_{1}\right|$ on the $\Xi$-support of $\sigma_{1}$ to see that for each term in the Littlewood-Paley decomposition, we can introduce for free a second Littlewood-Paley cutoff function in the variable $\xi_{1}+\cdots+\xi_{N}$ (that is, the 'dual' frequency variable). The same support property allows us to also introduce low-frequency cutoff operators (written later as $\left.P_{k}^{u_{j}}\right)$ in each of the $\xi_{j}$-variables $(j=2, \ldots, N)$ which restrict $\left|\xi_{j}\right| \lesssim 2^{k}$ when $\left|\xi_{1}\right| \approx 2^{k}$. For this purpose, it is more useful to have the squares of the functions form a partition of unity than the functions themselves - that is, equation (40) holds instead of equation (9). So although the $\psi_{k}$ in the following construction are essentially a Littlewood-Paley partition of unity in the sense of Definition 2.1, we emphasise that they depart slightly from that definition.

We introduce a positive, radial, radially decreasing, smooth cutoff function $\vartheta: \mathbb{R}^{n} \rightarrow \mathbb{R}$ which satisfies $\vartheta(\xi)=1$ if $|\xi| \leqslant 1$ and $\vartheta(\xi)=0$ if $|\xi| \geqslant 2$; and we define the nonnegative functions $\theta_{k}, \psi_{k}$ and $\phi_{k}$ via the following relations: 
○ $\theta_{k}(\xi):=\vartheta\left(2^{3-k} \xi\right)$

$\circ \psi_{k}(\xi)^{2}:=\vartheta\left(2^{-1-k} \xi\right)^{2}-\vartheta\left(2^{2-k} \xi\right)^{2}$

$\circ \phi_{k}(\xi)^{2}:=\vartheta\left(2^{-3-k} \xi\right)^{2}-\vartheta\left(2^{4-k} \xi\right)^{2}$.

Using the support properties of these functions, it is easy to verify the following facts:

(i) $\psi_{k}(\xi)=1$ for $2^{k-1} \leqslant|\xi| \leqslant 2^{k+1}$.

(ii) $\psi_{k}(\xi)=0$ for $|\xi| \leqslant 2^{k-2}$ and $2^{k+2} \leqslant|\xi|$.

(iii) $\theta_{k}(\eta)=1$ for $|\eta| \leqslant 2^{k-3}$.

(iv) $\theta_{k}(\eta)=0$ for $2^{k-2} \leqslant|\eta|$.

(v) $\phi_{k}(\xi+\eta)=1$ for $2^{k-3} \leqslant|\xi+\eta| \leqslant 2^{k+3}$.

(vi) $\phi_{k}(\xi+\eta)=0$ for $|\xi+\eta| \leqslant 2^{k-4}$ and $2^{k+4} \leqslant|\xi+\eta|$.

Given the support properties of $\sigma_{1}$, it follows that if $\psi_{k}\left(\xi_{1}\right) \neq 0$ and $\sigma_{1}(x, \Xi) \neq 0$, then

$$
\left|2^{-k} \Xi_{1}^{\prime}\right| \leqslant \frac{\left|2^{-k} \xi_{1}\right|}{32 \sqrt{N-1}} \leqslant \frac{2^{2}}{32 \sqrt{N-1}}=\frac{2^{-3}}{\sqrt{N-1}},
$$

which implies that $\theta_{k}\left(\xi_{j}\right)=1$ for $j=2, \ldots, N$.

Likewise, when $\psi_{k}\left(\xi_{1}\right) \neq 0$ and $\sigma_{1}(x, \Xi) \neq 0$, then

$$
\begin{aligned}
\frac{1}{4}-\frac{1}{8} \leqslant & \left|2^{-k} \xi_{1}\right|-\sqrt{N-1}\left|2^{-k} \Xi_{1}^{\prime}\right| \\
& \leqslant\left|2^{-k}\left(\xi_{1}+\cdots+\xi_{N}\right)\right| \leqslant\left|2^{-k} \xi_{1}\right|+\sqrt{N-1}\left|2^{-k} \Xi_{1}^{\prime}\right| \leqslant 4+\frac{1}{8}<8 \\
& \text { which implies } \phi_{k}\left(\xi_{1}+\cdots+\xi_{N}\right)=1 .
\end{aligned}
$$

Observe that on the support of $\sigma_{1}$,

$$
|\Xi|^{2}=\left|\xi_{1}\right|^{2}+\left|\Xi_{1}^{\prime}\right|^{2} \geqslant 1 / 64, \quad\left|\xi_{1}\right|^{2} \geqslant 32^{2}(N-1)\left|\Xi_{1}^{\prime}\right|^{2} .
$$

Then

$$
1 / 64 \leqslant\left(1+\frac{1}{32^{2}(N-1)}\right)\left|\xi_{1}\right|^{2}
$$

and so

$$
\left|\xi_{1}\right|^{2} \geqslant \frac{16(N-1)}{1+32^{2}(N-1)}>0 .
$$

Finally, it follows directly from the definition before that each function $\psi_{k}$ is radial and real-valued, and

$$
\sum_{k=-\infty}^{\infty} \psi_{k}(\xi)^{2}=1 \quad \text { for all } \xi \neq 0
$$

Using these facts, there exists $k_{0} \in \mathbb{Z}$ (independent of $x$ ) such that we can write $T_{\sigma_{1}}^{\Phi}$ as

$$
\begin{aligned}
& T_{\sigma_{1}}^{\Phi}\left(f_{1}, \ldots, f_{N}\right)(x) \\
& =\int_{\mathbb{R}^{n N}} \sum_{k \geqslant k_{0}} \psi_{k}\left(\xi_{1}\right)^{2} \prod_{j=2}^{N} \theta_{k}\left(\xi_{j}\right)^{2} \phi_{k}\left(\xi_{1}+\cdots+\xi_{N}\right)^{2} \sigma_{1}(x, \Xi) \widehat{f}_{1}\left(\xi_{1}\right) \prod_{j=2}^{N} \widehat{f}_{j}\left(\xi_{j}\right) e^{i x \cdot\left(\xi_{1}+\cdots+\xi_{N}\right)} e^{i \Phi(\Xi)} \mathrm{d} \Xi,
\end{aligned}
$$


which by setting

$$
\Phi(x, \Xi):=x \cdot\left(\xi_{1}+\cdots+\xi_{N}\right)+\varphi_{0}\left(\xi_{1}+\cdots+\xi_{N}\right)+\sum_{j=1}^{N} \varphi_{j}\left(\xi_{j}\right)
$$

we can in turn write as

$$
\begin{aligned}
& \int_{\mathbb{R}^{n N}} \sum_{k \geqslant k_{0}}^{\infty} \mathfrak{a}\left(k, x, 2^{-k} \Xi\right)\left[\left|\xi_{1}+\cdots+\xi_{N}\right|^{m_{0}} \phi_{k}\left(\xi_{1}+\cdots+\xi_{N}\right)\right] \\
& \quad \times\left[\left|\xi_{1}\right|^{m_{1}}\left|2^{-k} \xi_{1}\right|^{m-m_{0}-m_{1}} \psi_{k}\left(\xi_{1}\right) \widehat{f}\left(\xi_{1}\right)\right]\left[\prod_{j=2}^{N} 2^{k m_{j}} \theta_{k}\left(\xi_{j}\right)^{2} \widehat{f}_{j}\left(\xi_{j}\right)\right] e^{i \Phi(x, \Xi)} \mathrm{d} \Xi,
\end{aligned}
$$

where $m=\sum_{j=0}^{N} m_{j}$ and

$$
\mathfrak{a}(k, x, \Xi)=\sigma_{1}\left(x, 2^{k} \Xi\right) \psi_{1}\left(\xi_{1}\right) \prod_{j=2}^{N} \theta_{1}\left(\xi_{j}\right) \phi_{1}\left(\xi_{1}+\cdots+\xi_{N}\right)\left(\frac{2^{-k}}{\left|\xi_{1}\right|}\right)^{m-m_{0}}\left(\frac{2^{-k}}{\left|\xi_{1}+\cdots+\xi_{N}\right|}\right)^{m_{0}} .
$$

If we introduce a high-frequency cutoff $\chi_{0}$ that satisfies

$\circ \chi_{0}(\xi)=1$ for $|\xi| \geqslant 2^{k_{0}-4}$ and

○ $\chi_{0}(\xi)=0$ for $|\xi| \leqslant 2^{k_{0}-5}$,

we can use formula (39) and facts (i) and (ii) before it to rewrite formula (43) as

$$
\begin{aligned}
& \int_{\mathbb{R}^{n N}} \sum_{k \geqslant k_{0}}^{\infty} \mathfrak{a}\left(k, x, 2^{-k} \Xi\right)\left[\left|\xi_{1}+\cdots+\xi_{N}\right|^{m_{0}} \chi_{0}\left(\xi_{1}+\cdots+\xi_{N}\right) \phi_{k}\left(\xi_{1}+\ldots+\xi_{N}\right)\right] \times \\
& {\left[\left|\xi_{1}\right|^{m_{1}}\left|2^{-k} \xi_{1}\right|^{m-m_{0}-m_{1}} \chi_{0}\left(\xi_{1}\right) \psi_{k}\left(\xi_{1}\right) \widehat{f}\left(\xi_{1}\right)\right]\left[\prod_{j=2}^{N} 2^{k m_{j}} \theta_{k}\left(\xi_{j}\right) \widehat{f}_{j}\left(\xi_{j}\right)\right] e^{i \Phi(x, \Xi)} \mathrm{d} \Xi .}
\end{aligned}
$$

Making use of the Fourier inversion formula, we can write

$$
\mathfrak{a}(k, x, \Xi)=\int \frac{\mathrm{m}(k, x, U)}{\left(1+|U|^{2}\right)^{M}} e^{i \Xi \cdot U} \mathrm{~d} U, \quad U=\left(u_{1}, \ldots, u_{N}\right),
$$

for a smooth bounded function $m$. This means we can then write $T_{\sigma_{1}}^{\Phi}\left(f_{1}, \ldots, f_{N}\right)(x)$ as a weighted average in $U$ of

$$
\begin{aligned}
\sum_{k=k_{0}}^{\infty} \mathrm{m}(k, x, U) \int\left[\left|\xi_{1}+\cdots+\xi_{N}\right|^{m_{0}} \phi_{k}\left(\xi_{1}+\ldots+\xi_{N}\right) \chi_{0}\left(\xi_{1}+\ldots+\xi_{N}\right)\right] \times \\
\quad\left[\left|\xi_{1}\right|^{m_{1}}\left|2^{-k} \xi_{1}\right|^{m-m_{0}-m_{1}} \chi_{0}\left(\xi_{1}\right) \psi_{k}\left(\xi_{1}\right) e^{i 2^{-k}} \xi_{1} \cdot u_{1} \widehat{f}_{1}(\xi)\right] \times \\
{\left[\prod_{j=2}^{N} 2^{k m_{j}} \theta_{k}\left(\xi_{j}\right) \widehat{f}_{j}\left(\xi_{j}\right) e^{i 2^{-k} \xi_{j} \cdot u_{j}}\right] e^{i \Phi(x, \Xi)} \mathrm{d} \Xi . }
\end{aligned}
$$


Finally, we can write formula (44) as

$$
B\left(f_{1}, \ldots, f_{N}\right)(x)=\sum_{k=k_{0}}^{\infty} M_{\mathfrak{m}} \circ T_{b_{0}}^{\varphi_{0}} \circ Q_{k}^{0}\left[\left(Q_{k}^{u_{1}} \circ T_{b_{1}}^{\varphi_{1}}\right)\left(f_{1}\right) \prod_{j=2}^{N}\left(P_{k}^{u_{j}} \circ T_{b_{j}}^{\varphi_{j}}\right)\left(f_{j}\right)\right](x),
$$

where

$$
\begin{array}{lrl}
\widehat{Q_{k}^{0}(f)}(\xi)=\phi_{k}(\xi) \widehat{f}(\xi), & b_{0}(\xi)=|\xi|^{m_{0}} \chi_{0}(\xi), \\
\widehat{Q_{k}^{u_{1}}(f)}(\xi)=\left|2^{-k} \xi\right|^{m-m_{0}-m_{1}} \psi_{k}(\xi) e^{i 2^{-k} \xi \cdot u_{1}} \widehat{f}(\xi), & b_{1}(\xi)=|\xi|^{m_{1}} \chi_{0}(\xi), \\
\overline{P_{k}^{u_{j}}(f)}(\xi)=\theta_{k}(\xi) e^{i 2^{-k} \xi \cdot u_{j}} \widehat{f}(\xi), & b_{j}(k, \xi)=2^{k m_{j}} \omega_{k}(\xi),
\end{array}
$$

for $j=2, \ldots, N ; \omega_{k}(\xi):=\theta_{k}(\xi / 2)$ is a bump function equal to 1 on the support of $\theta_{k}$; and $M_{\mathfrak{m}}$ denotes multiplication by $\mathrm{m}^{1}$

The position of the operator $M_{\mathfrak{m}}$ and the fact that $\mathrm{m}$ depends on both $k$ and $x$ cause problems if we wish to make use of various square-function and Carleson-measure estimates to estimate norms of equation (45). We can overcome the problems by observing that this dependency is in fact periodic. Indeed, since $Q_{k}^{0}=\left(Q_{k-1}^{0}+Q_{k}^{0}+Q_{k+1}^{0}\right) \circ Q_{k}^{0}$ and $Q_{k}^{0} \circ Q_{k^{\prime}}^{0} \equiv 0$ if $\left|k-k^{\prime}\right| \geqslant 2$, we can write

$$
M_{\mathfrak{m}} \circ T_{b_{0}}^{\varphi_{0}} \circ Q_{k}^{0}=\left(\sum_{k^{\prime}=k-1}^{k+1} T_{k, k^{\prime}}^{U}\right) \circ Q_{k}^{0}=\left(\sum_{\ell=-1}^{1} \sum_{k^{\prime}-k \equiv \ell(\bmod 3)} T_{k^{\prime}+\ell, k^{\prime}}^{U}\right) \circ Q_{k}^{0},
$$

where $T_{j, k}^{U}$ is the FIO with amplitude $\mathfrak{m}(j, x, U) b_{0}(\xi) \phi_{k}(\xi)$ and phase $\varphi_{0}$. Observe that

$$
\mathcal{T}_{k}^{U}:=\sum_{\ell=-1}^{1} \sum_{k^{\prime}-k \equiv \ell(\bmod 3)} T_{k^{\prime}+\ell, k^{\prime}}^{U}
$$

is periodic in $k$ with period 3, and is an FIO with amplitude in $S^{m_{0}}$. Thus, equation (45) can be rewritten as

$$
\sum_{\ell=0}^{2} \mathcal{T}_{\ell}^{U}\left(B_{\ell}\left(f_{1}, \ldots, f_{N}\right)\right)(x)
$$

where

$$
\begin{aligned}
& B_{\ell}\left(f_{1}, \ldots, f_{N}\right)(x) \\
& :=\sum_{k \equiv \ell(\bmod 3), k \geqslant k_{0}} \chi_{0}(2 D) Q_{k}^{0}\left[\left(Q_{k}^{u_{1}} \circ T_{b_{1}}^{\varphi_{1}}\right)\left(f_{1}\right) \prod_{j=2}^{N}\left(P_{k}^{u_{j}} \circ T_{b_{j}}^{\varphi_{j}}\right)\left(f_{j}\right)\right](x),
\end{aligned}
$$

and $\chi_{0}$ is the same high-frequency cutoff introduced before (and is a symbol belonging to $S^{0}$ ). Now, by Theorem 3.1, each $\mathcal{T}_{\ell}^{U}$ is a bounded operator on $X^{p}$ (with norms uniform in $U$ ), and so the boundedness of $T_{\sigma_{1}}^{\Phi}$ is reduced to studying the boundedness of $B_{\ell}$. In the remainder of this section, we prove this boundedness in each of the endpoint cases from Corollary 5.2. Due to the symmetry of definition (46) in the indices $j=2, \ldots, N$, we only need to consider endpoint cases $\left(p_{0}, \ldots, p_{N}\right)$ which are distinct within the equivalence class of permutations of $\left(p_{2}, \ldots, p_{N}\right)$.

\footnotetext{
${ }^{1}$ The notation $Q_{k}^{0}$ and $Q_{k}^{u_{1}}$ is potentially ambiguous, as $\left.Q_{k}^{u_{1}}\right|_{u_{1}=0}$ is not the same operator as $Q_{k}^{0}$. However, in practice no confusion need arise, so to avoid a profusion of notation, we tolerate this imprecision.
} 


\subsection{Boundedness with the target space $L^{2}$}

In this case we take $m_{0}=0$. By duality and definition (46), we have

$$
\begin{aligned}
& \left\|B_{\ell}\left(f_{1}, \ldots, f_{N}\right)\right\|_{L^{2}} \\
= & \sup _{\left\|f_{0}\right\|_{L^{2}}=1}\left|\int f_{0}(x) B_{\ell}\left(f_{1}, \ldots, f_{N}\right)(x) \mathrm{d} x\right| \\
= & \sup _{\left\|f_{0}\right\|_{L^{2}}=1}\left|\sum_{k \equiv \ell(\bmod 3), k \geqslant k_{0}} \int Q_{k}^{0}\left(\chi_{0}(2 D) f_{0}\right)(x)\left(Q_{k}^{u} \circ T_{b_{1}}^{\varphi_{1}}\right)\left(f_{1}\right)(x) \prod_{j=2}^{N}\left(P_{k}^{u_{j}} \circ T_{b_{j}}^{\varphi_{j}}\right)\left(f_{j}\right)(x) \mathrm{d} x\right| \\
\leqslant & \sup _{\left\|f_{0}\right\|_{L^{2}=1}}\left(\sum_{k \equiv \ell(\bmod 3), k \geqslant k_{0}} \int\left|Q_{k}^{0}\left(\chi_{0}(2 D) f_{0}\right)(x)\right|^{2} \mathrm{~d} x\right)^{1 / 2} \\
& \times\left(\sum_{k \equiv \ell(\bmod 3), k \geqslant k_{0}} \int\left|\left(Q_{k}^{u} \circ T_{b_{1}}^{\varphi_{1}}\right)\left(f_{1}\right)(x) \prod_{j=2}^{N}\left(P_{k}^{u_{j}} \circ T_{b_{j}}^{\varphi_{j}}\right)\left(f_{j}\right)\right|^{2} \mathrm{~d} x\right)^{1 / 2} .
\end{aligned}
$$

For the first factor we just use the quadratic estimate

$$
\left(\sum_{k \equiv \ell(\bmod 3), k \geqslant k_{0}} \int\left|Q_{k}^{0}\left(\chi_{0}(2 D) f_{0}\right)(x)\right|^{2} \mathrm{~d} x\right)^{1 / 2} \lesssim\left\|\chi_{0}(2 D) f_{0}\right\|_{L^{2}} \lesssim 1 .
$$

Thus it remains to control

$$
\left(\sum_{k \equiv \ell(\bmod 3), k \geqslant k_{0}} \int\left|\left(Q_{k}^{u_{1}} \circ T_{b_{1}}^{\varphi_{1}}\right)\left(f_{1}\right)(x) \prod_{j=2}^{N}\left(P_{k}^{u_{j}} \circ T_{b_{j}}^{\varphi_{j}}\right)\left(f_{j}\right)\right|^{2} \mathrm{~d} x\right)^{1 / 2},
$$

and precisely how this is done depends on the endpoint case considered, so we consider each case in turn.

\subsection{1. bmo $\times \cdots \times$ bmo $\times L^{2} \rightarrow L^{2}$}

Here we take $n \geqslant 2, m_{j}=-(n-1) / 2, f_{j} \in$ bmo for $j=1, \ldots, N-1, m_{N}=0$ and $f_{N} \in L^{2}$. By Theorem 3.1 we know that $T_{b_{1}}^{\varphi_{1}}\left(f_{1}\right) \in$ BMO when $f_{1} \in$ bmo. This implies that

$$
\mathrm{d} \mu(x, t)=\sum_{k \in \mathbb{Z}}\left|\left(Q_{k}^{u_{1}} \circ T_{b_{1}}^{\varphi_{1}}\right)\left(f_{1}\right)(x)\right|^{2} \mathrm{~d} x \delta_{2^{-k}}(t),
$$

where $\delta_{2^{-k}}$ is a Dirac mass at the point $2^{-k}$, is a Carleson measure with the Carleson norm bounded by a constant multiple of $\left\|f_{1}\right\|_{\mathrm{bmo}}$. Moreover, the nontangential maximal function of $(x, t) \mapsto\left(P_{k}^{u_{N}} \circ T_{b_{N}}^{\varphi_{N}}\right)\left(f_{N}\right)(x) \delta_{2^{-k}}(t)$ is in $L^{2}$ when $f_{N} \in L^{2}$. Thus, to control formula (47) with formula (15) and conclude the proof in this endpoint case, it is enough to apply formula (49) from the following lemma to $P_{k}^{u_{j}} \circ T_{b_{j}}^{\varphi_{j}}$ for each $j=2, \ldots, N-1$.

Lemma 7.1. Let

$$
m=-(n-1)\left|\frac{1}{p}-\frac{1}{2}\right|, \quad n /(n+1)<p \leqslant \infty .
$$

Let

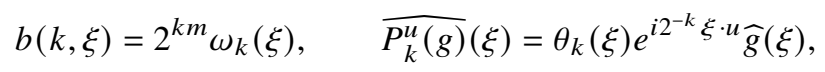


where $\omega_{k}$ and $\theta_{k}$ are the cutoff functions already defined. It follows that

$$
\sup _{k}\left\|\left(P_{k}^{u} \circ T_{b}^{\varphi}\right)(f)\right\|_{h^{p}} \lesssim\|f\|_{h^{p}}
$$

and for $n \geqslant 2$ we also have, for $m=-(n-1) / 2$,

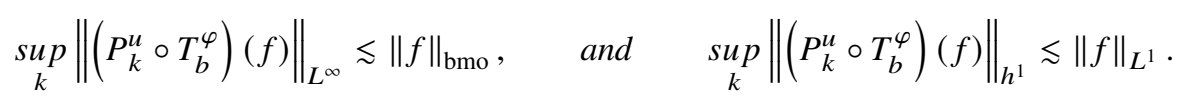

Proof. The proof of formula (48) follows from the fact that the amplitude of $P_{k}^{u} \circ T_{b}^{\varphi}$ is in $S^{m}$ uniformly in $k$. By duality, self-adjointness of the operators involved and interpolation, the second inequality in formula (49) follows from the first.

In order to establish the first inequality in formula (49), we write $b=b^{b}+b^{\sharp}$, where

$$
b^{b}(k, \xi)=b(k, \xi)\left(1-\chi_{0}(\xi)\right) \quad \text { and } \quad b^{\sharp}(k, \xi)=b(k, \xi) \chi_{0}(\xi) .
$$

Now since $m \leqslant 0$ and $1-\chi_{0}$ is a low frequency cutoff, we can throw away the $\omega$ in the definition of $b$, which would then eliminate the $k$-dependency in $b^{b}$. Then by the kernel estimates for the FIOs with amplitude $b^{b}$ (see, for example, Lemma 2.3), for $f \in$ bmo we have

$$
\left\|P_{k}^{u} T_{b^{b}}^{\varphi}(f)\right\|_{L^{\infty}} \lesssim\left\|T_{b^{b}}^{\varphi}(f)\right\|_{L^{\infty}} \lesssim\left\|\left(1-\chi_{0}\right)(D) f\right\|_{L^{\infty}} \lesssim\|f\|_{\mathrm{bmo}} .
$$

In order to ameliorate $\left(P_{k}^{u} \circ T_{b^{\sharp}}^{\varphi}\right)(f)$ so that we can better understand its action on bmo functions, we employ an argument from [21, page 27]. According to that argument, for $n \geqslant 2$ and $m=\frac{-(n-1)}{2}$ we introduce an operator

$$
R_{k}=\sum_{j=k_{0}}^{k} Q_{j} 2^{(k-j) m}
$$

with some positive $k_{0}$, which enables us to replace $\left(P_{k}^{u} \circ T_{b^{\sharp}}^{\varphi}\right)(f)$ by $P_{k}^{u} \circ R_{k} \circ T_{\gamma}^{\varphi}(f)$ for $n \geqslant 2$, where $\gamma(\xi):=\chi_{0}(\xi)|\xi|^{m} \in S^{-(n-1) / 2}$.

By [21, Lemma 4.8], the operator $R_{k}$ has a kernel $K_{k}$ which has the following properties:

$$
\int K_{k}(z) \mathrm{d} z=0
$$

and for each $0<\delta<\frac{n-1}{2}$, the estimates

$$
\left|K_{k}(x-y)\right| \lesssim 2^{k n}\left(1+\frac{|x-y|}{2^{-k}}\right)^{-n-\delta}
$$

and

$$
\left|K_{k}(x-y)-K_{k}\left(x-y^{\prime}\right)\right| \lesssim 2^{k(n+1)}\left|y-y^{\prime}\right|
$$

hold for all $x, y, y^{\prime} \in \mathbb{R}^{n}$ and $k \in \mathbb{Z}$. Therefore $R_{k}$ satisfies

$$
\sup _{k \in \mathbb{Z}}\left\|R_{k} f\right\|_{L^{q}} \lesssim\|f\|_{L^{q}}, \quad 1 \leqslant q<\infty,
$$

and

$$
\sup _{k \in \mathbb{Z}}\left\|R_{k} f\right\|_{L^{\infty}} \lesssim\|f\|_{\mathrm{BMO}} .
$$


Using this BMO- $L^{\infty}$-boundedness (valid for $n \geqslant 2$ ), the global bmo-boundedness of FIOs with amplitudes in $S^{-(n-1) / 2}$ (Theorem 3.1) and the $L^{\infty}$-boundedness of $P_{k}^{u}$ yield

$$
\underset{k}{\sup }\left\|P_{k}^{u} T_{b^{\sharp}}^{\varphi}(f)\right\|_{L^{\infty}} \lesssim\left\|\chi_{0}(D) f\right\|_{\mathrm{BMO}} \leqslant\|f\|_{\mathrm{bmo}} .
$$

Remark 7.2. Here we see that the assumption $n \geqslant 2$ is used in the proof of Lemma 7.1 to ensure that $\delta$ can be chosen positive. This is not just a feature of the proof, but is in fact necessary. As was shown in [21, Proposition 5.3], the bilinear operator in dimension $n=1$ with amplitude $\sigma \equiv 1$ and phase functions $\varphi_{1}=x \xi+|\xi|, \varphi_{2}=x \eta$ and $\varphi_{3}=0$ fails to be bounded from bmo $\times L^{2}$ to $L^{2}$.

\subsection{2. $L^{2} \times$ bmo $\times \cdots \times$ bmo $\rightarrow L^{2}$}

Here we take $m_{1}=0, f_{1} \in L^{2}, m_{j}=-(n-1) / 2$ and $f_{j} \in$ bmo for $j=2, \ldots, N$. Noting that $b_{1}$ does not depend on $k$, the quadratic estimate

$$
\left(\sum_{k \equiv \ell(\bmod 3), k \geqslant k_{0}} \int\left|\left(Q_{k}^{u_{1}} \circ T_{b_{1}}^{\varphi_{1}}\right)\left(f_{1}\right)(x)\right|^{2} \mathrm{~d} x\right)^{1 / 2} \lesssim\left\|T_{b_{1}}^{\varphi_{1}}\left(f_{1}\right)\right\|_{L^{2}} \lesssim\left\|f_{1}\right\|_{L^{2}}
$$

follows with the help of formula (17) and Theorem 3.1. Applying this and formula (49) to expression (47) yields

$$
\begin{aligned}
& \left(\sum_{k \equiv \ell(\bmod 3), k \geqslant k_{0}} \int\left|\left(Q_{k}^{u_{1}} \circ T_{b_{1}}^{\varphi_{1}}\right)\left(f_{1}\right)(x) \prod_{j=2}^{N}\left(P_{k}^{u_{j}} \circ T_{b_{j}}^{\varphi_{j}}\right)\left(f_{j}\right)\right|^{2} \mathrm{~d} x\right)^{1 / 2} \\
& \lesssim\left(\sum_{k \equiv \ell(\bmod 3), k \geqslant k_{0}} \int\left|\left(Q_{k}^{u_{1}} \circ T_{b_{1}}^{\varphi_{1}}\right)\left(f_{1}\right)(x)\right|^{2} \mathrm{~d} x\right)^{1 / 2} \prod_{j=2}^{N} \sup \left\|\left(P_{k}^{u_{j}} \circ T_{b_{j}}^{\varphi_{j}}\right)\left(f_{j}\right)\right\|_{L^{\infty}} \\
& \leqslant\left\|f_{1}\right\|_{L^{2}} \prod_{j=2}^{N}\left\|f_{j}\right\|_{\mathrm{bmo}} .
\end{aligned}
$$

\subsection{Boundedness with the target space $h^{1}$}

Now we take $m_{0}=-(n-1) / 2$, and so by duality and definition (46) we have

$$
\begin{aligned}
& \left\|B_{\ell}\left(f_{1}, \ldots, f_{N}\right)\right\|_{h^{1}}= \\
& \sup _{\left\|f_{0}\right\|_{\mathrm{bmo}}=1} \sum_{k \equiv \ell(\bmod 3), k \geqslant k_{0}} \int Q_{k}^{0}\left(\chi_{0}(2 D) f_{0}\right)\left(Q_{k}^{u_{1}} \circ T_{b_{1}}^{\varphi_{1}}\right)\left(f_{1}\right) \prod_{j=2}^{N}\left(P_{k}^{u_{j}} \circ T_{b_{j}}^{\varphi_{j}}\right)\left(f_{j}\right) \mathrm{d} x \mid .
\end{aligned}
$$

Since $f_{0} \in$ bmo, we have that $\chi_{0}(2 D) f_{0} \in$ BMO. Therefore,

$$
\mathrm{d} \mu_{f_{0}}(x, t):=\sum_{k \equiv \ell(\bmod 3), k \geqslant k_{0}}\left|Q_{k}^{0}\left(\chi_{0}(2 D) f_{0}\right)(x)\right|^{2} \mathrm{~d} x \delta_{2^{-k}}(t)
$$

is a Carleson measure with Carleson norm not exceeding a constant multiple of $\left\|f_{0}\right\|_{\mathrm{bmo}}^{2}$.

7.2.1. bmo $\times \cdots \times$ bmo $\times h^{1} \rightarrow h^{1}$

Here we take $m_{j}=-(n-1) / 2$ for $j=0, \ldots N, f_{j} \in$ bmo, $j=0, \ldots, N-1$, and $f_{N} \in h^{1}$ in equation (52). 
Since $f_{1} \in$ bmo, Theorem 3.1 and definition (11) yield that $T_{b_{1}}^{\varphi_{1}}\left(f_{1}\right) \in \mathrm{BMO}$, and therefore

$$
\mathrm{d} \mu_{f_{1}}(x, t):=\sum_{k \equiv \ell(\bmod 3), k \geqslant k_{0}}\left|Q_{k}^{u} \circ T_{b_{1}}^{\varphi_{1}}\left(f_{1}\right)(x)\right|^{2} \mathrm{~d} x \delta_{2^{-k}}(t)
$$

is a Carleson measure. Since we also have

$$
\begin{aligned}
& \left|Q_{k}^{0} \circ \chi_{0}(2 D)\left(f_{0}\right)(x)\right|\left|Q_{k}^{u_{1}} \circ T_{b_{1}}^{\varphi_{1}}\left(f_{1}\right)(x)\right| \\
& \leqslant \frac{1}{2}\left(\frac{\left\|f_{1}\right\|_{\mathrm{bmo}}}{\left\|f_{0}\right\|_{\mathrm{bmo}}}\left|Q_{k}^{0} \circ \chi_{0}(2 D)\left(f_{0}\right)(x)\right|^{2}+\frac{\left\|f_{0}\right\|_{\mathrm{bmo}}}{\left\|f_{1}\right\|_{\mathrm{bmo}}}\left|Q_{k}^{u_{1}} \circ T_{b_{1}}^{\varphi_{1}}\left(f_{1}\right)(x)\right|^{2}\right),
\end{aligned}
$$

the measure

$$
\mathrm{d} \mu_{f_{0}, f_{1}}(x, t):=\sum_{k \equiv \ell(\bmod 3), k \geqslant k_{0}} Q_{k}^{0} \circ \chi_{0}(2 D)\left(f_{0}\right)(x) Q_{k}^{u_{1}} \circ T_{b_{1}}^{\varphi_{1}}\left(f_{1}\right)(x) \mathrm{d} x \delta_{2^{-k}}(t)
$$

is also Carleson, with Carleson norm bounded by $\left\|f_{0}\right\|_{\mathrm{bmo}}\left\|f_{1}\right\|_{\mathrm{bmo}}$. Moreover, by formula (49), even

$$
\mathrm{d} \mu_{f_{0}, \ldots, f_{N-1}}(x, t):=\sum_{k} \prod_{j=2}^{N-1}\left(P_{k}^{u_{j}} \circ T_{b_{j}}^{\varphi_{j}}\right)\left(f_{j}\right)(x) \delta_{2^{-k}}(t) \mathrm{d} \mu_{f_{0}, f_{1}}(x, t)
$$

is a Carleson measure.

At this point we repeat the decomposition (50) of $b_{N}$ into the sum $b_{N}^{b}+b_{N}^{\sharp}$. We can see that since $m_{N}=-(n-1) / 2$ and $1-\chi_{0}$ is a low-frequency cutoff, we can disregard the $\omega_{k}$ in the definition of $b_{N}^{b}$, which means $b_{N}^{b}$ is independent of $k$. Then the characterisation of local Hardy spaces in Definition 2.2(v) and formula (14) yield

$$
\begin{aligned}
& \left|\sum_{k \equiv \ell(\bmod 3), k \geqslant k_{0}} \int\left(P_{k}^{u_{N}} \circ T_{b_{N}^{\mathrm{b}}}^{\varphi_{N}}\right)\left(f_{N}\right) \mathrm{d} \mu_{f_{0}, \ldots, f_{N-1}}\left(x, 2^{-k}\right)\right| \\
& \lesssim \prod_{j=0}^{N-1}\left\|f_{j}\right\|_{\mathrm{bmo}} \int_{\mathbb{R}^{n}} \sup _{k \geqslant k_{0}|x-y|<2^{-k}} \sup _{\mid{ }^{\prime}}\left|\left(P_{k}^{u_{N}} \circ T_{b_{N}^{\mathrm{b}}}^{\varphi_{N}}\right)\left(f_{N}\right)\right| \mathrm{d} x \\
& \lesssim\left\|T_{b_{N}^{\mathrm{b}}}^{\varphi_{N}}\left(f_{N}\right)\right\|_{h^{1}} \prod_{j=0}^{N-1}\left\|f_{j}\right\|_{\mathrm{bmo}} \lesssim \prod_{j=0}^{N-1}\left\|f_{j}\right\|_{\mathrm{bmo}}\left\|f_{N}\right\|_{h^{1}} .
\end{aligned}
$$

To deal with $\left(P_{k}^{u_{N}} \circ T_{b_{N}^{\sharp}}^{\varphi_{N}}\right)\left(f_{N}\right)$, we continue to follow the proof of Lemma 7.1 and replace it with $P_{k}^{u_{N}} \circ R_{k}^{N} \circ T_{\gamma}^{\varphi_{N}}\left(f_{N}\right)$, where $\gamma \in S^{m_{N}}$. Lemma 2.6 leads us to conclude that

$$
\sum_{k} R_{k}^{N *}\left(\mathrm{~d} \mu_{f_{0}, \ldots, f_{N-1}}\left(\cdot, 2^{-k}\right)\right)(x) \delta_{2^{-k}}(t) \mathrm{d} x
$$

\footnotetext{
${ }^{2}$ This is necessary because $b_{N}$ depends on $k$. If it did not, the proof of this endpoint could be completed by arguing as in formula (54) directly with $b_{N}$ instead of $b_{N}^{b}$.
} 
is also a Carleson measure. This, via formula (16), yields

$$
\begin{aligned}
& \left|\sum_{k \equiv \ell(\bmod 3), k \geqslant k_{0}} \int\left(P_{k}^{u_{N}} \circ T_{b_{N}^{\sharp}}^{\varphi_{N}}\right)\left(f_{N}\right) \mathrm{d} \mu_{f_{0}, \ldots, f_{N-1}}\left(x, 2^{-k}\right)\right| \\
& =\left|\sum_{k \equiv \ell(\bmod 3), k \geqslant k_{0}} \int\left(P_{k}^{u_{N}} \circ T_{\gamma}^{\varphi_{N}}\right)\left(f_{N}\right) R_{k}^{N *}\left(\mathrm{~d} \mu_{f_{0}, \ldots, f_{N-1}}\left(\cdot, 2^{-k}\right)\right) \mathrm{d} x\right| \\
& \lesssim\left\|T_{\gamma}^{\varphi_{N}}\left(f_{N}\right)\right\|_{h^{1}} \prod_{j=0}^{N-1}\left\|f_{j}\right\|_{\mathrm{bmo}} \lesssim \prod_{j=0}^{N-1}\left\|f_{j}\right\|_{\mathrm{bmo}}\left\|f_{N}\right\|_{h^{1}} .
\end{aligned}
$$

\subsection{2. $h^{1} \times$ bmo $\times \cdots \times$ bmo $\rightarrow h^{1}$}

Here we take $m_{j}=-(n-1) / 2$, for $j=0, \ldots, N, f_{0} \in$ bmo, $f_{1} \in h^{1}$ and $f_{j} \in$ bmo, $j=2, \ldots, N$. Using formula (49), from Lemma 7.1 we have

$$
\sup _{k}\left\|P_{k}^{u_{j}} T_{b_{j}}^{\varphi_{j}}\left(f_{j}\right)\right\|_{L^{\infty}} \lesssim\left\|f_{j}\right\|_{\mathrm{bmo}}, \quad j=2, \ldots, N .
$$

We now take

$$
\begin{aligned}
& G(x)=\chi_{0}(2 D)\left(f_{0}\right)(x), \quad v\left(2^{-k}, x\right)=\prod_{j=2}^{\infty} P_{k}^{u_{j}} \circ T_{b_{j}}^{\varphi_{j}}\left(f_{j}\right), \quad \text { and } \\
& F(x)=\left(Q_{k}^{u_{1}} \circ T_{b_{1}}^{\varphi_{1}}\right)\left(f_{1}\right)=\left(Q_{k}^{u_{1}} \circ T_{b_{1}}^{\varphi_{1}} \circ \chi_{0}(2 D)\right)\left(f_{1}\right),
\end{aligned}
$$

and thereafter apply Proposition 2.7, Theorem 3.1 and definition (11) to the right-hand side of equation (52) to obtain

$$
\begin{aligned}
\sup _{\left\|f_{0}\right\|_{\mathrm{bmo}}=1} \mid & \sum_{k \equiv \ell(\bmod 3), k \geqslant k_{0}} \int Q_{k}^{0}\left(\chi_{0}(2 D) f_{0}\right)\left(Q_{k}^{u_{1}} \circ T_{b_{1}}^{\varphi_{1}}\right)\left(\chi_{0}(2 D) f_{1}\right) \prod_{j=2}^{N}\left(P_{k}^{u_{j}} \circ T_{b_{j}}^{\varphi_{j}}\right)\left(f_{j}\right) \mathrm{d} x \mid \\
& \lesssim\left\|\chi_{0}(2 D) f_{1}\right\|_{H^{1}} \prod_{j=2}^{N}\left\|f_{j}\right\|_{\mathrm{bmo}} \lesssim\left\|f_{1}\right\|_{h^{1}} \prod_{j=2}^{N}\left\|f_{j}\right\|_{\mathrm{bmo}},
\end{aligned}
$$

where we have also used formula (10) in dealing with $\left\|\chi_{0}(2 D) f_{1}\right\|_{H^{1}}$.

7.2.3. $L^{2} \times L^{2} \times$ bmo $\times \cdots \times$ bmo $\rightarrow h^{1}$

We choose $m_{1}=m_{2}=0, f_{1}, f_{2} \in L^{2}, m_{j}=-\frac{n-1}{2}$ for $j=0$ and $j=3, \ldots N$ and $f_{0} \in$ bmo. Starting once again with equation (52), we have, for all $\left\|f_{0}\right\|_{\text {bmo }}=1$,

$$
\begin{aligned}
& \left|\sum_{k \equiv \ell(\bmod 3), k \geqslant k_{0}} \int Q_{k}^{0}\left(\chi_{0}(2 D) f_{0}\right)\left(Q_{k}^{u_{1}} \circ T_{b_{1}}^{\varphi_{1}}\right)\left(f_{1}\right) \prod_{j=2}^{N}\left(P_{k}^{u_{j}} \circ T_{b_{j}}^{\varphi_{j}}\right)\left(f_{j}\right) \mathrm{d} x\right| \\
& \lesssim\left(\int \sum_{k \geqslant k_{0}}\left|Q_{k}^{u_{1}} \circ T_{b_{1}}^{\varphi_{1}}\left(f_{1}\right)\right|^{2} \mathrm{~d} x\right)^{1 / 2} \\
& \left(\sum_{k \equiv \ell(\bmod 3), k \geqslant k_{0}} \int\left|P_{k}^{u_{2}} \circ T_{b_{2}}^{\varphi_{2}}\left(f_{2}\right)\right|^{2}\left|Q_{k}^{0}\left(\chi_{0}(2 D) f_{0}\right)\right|^{2} \prod_{j=3}^{N}\left|P_{k}^{u_{j}} \circ T_{b_{j}}^{\varphi_{j}}\left(f_{j}\right)\right|^{2} \mathrm{~d} x\right)^{1 / 2} .
\end{aligned}
$$


Since $f_{j} \in$ bmo for $j=0,3, \ldots, N$, we can argue as we did for definition (53) to conclude that

$$
\sum_{k \geqslant k_{0}}\left|Q_{k}^{0}\left(\chi_{0}(2 D) f_{0}\right)\right|^{2} \prod_{j=3}^{N}\left|P_{k}^{u_{j}} \circ T_{b_{j}}^{\varphi_{j}}\left(f_{j}\right)\right|^{2} \mathrm{~d} x \delta_{2^{-k}}(t)
$$

defines a Carleson measure, with Carleson norm bounded by $\left\|f_{0}\right\|_{\mathrm{bmo}}^{2} \prod_{j=3, \ldots, N}\left\|f_{j}\right\|_{\mathrm{bmo}}^{2}$. The $L^{2}$ boundedness of FIOs from Theorem 3.1, together with a quadratic estimate (17) in the first factor and a nontangential maximal function estimate (15) in the second, yields that formula (55) is bounded by

$$
\left\|f_{1}\right\|_{L^{2}} \times\left\|f_{2}\right\|_{L^{2}} \prod_{j=3}^{N}\left\|f_{j}\right\|_{\mathrm{bmo}}
$$

We would also like to note that when $N=2$, the functions $f_{j}$, with $j=3, \ldots, N$, do not appear in these estimates.

\subsection{4. bmo $\times L^{2} \times L^{2} \times$ bmo $\times \cdots \times$ bmo $\rightarrow h^{1}$}

We choose $m_{2}=m_{3}=0, f_{2}, f_{3} \in L^{2}, m_{j}=-\frac{n-1}{2}$ for $j=0,1,4, \ldots N$, and $f_{0}, f_{1}$ are both in bmo. Continuing from equation (52), we have, for all $\left\|f_{0}\right\|_{\mathrm{bmo}}=1$,

$$
\begin{aligned}
& \left|\sum_{k \equiv \ell(\bmod 3), k \geqslant k_{0}} \int Q_{k}^{0}\left(\chi_{0}(2 D) f_{0}\right)\left(Q_{k}^{u_{1}} \circ T_{b_{1}}^{\varphi_{1}}\right)\left(f_{1}\right) \prod_{j=2}^{N}\left(P_{k}^{u_{j}} \circ T_{b_{j}}^{\varphi_{j}}\right)\left(f_{j}\right) \mathrm{d} x\right| \\
& \lesssim\left(\int \sum_{k \geqslant k_{0}}\left|P_{k}^{u_{3}} T_{b_{3}}^{\varphi_{3}}\left(f_{3}\right)\right|^{2}\left|Q_{k}^{u_{1}} \circ T_{b_{1}}^{\varphi_{1}}\left(f_{1}\right)\right|^{2} \mathrm{~d} x\right)^{1 / 2} \\
& \quad \times\left(\sum_{k \equiv \ell(\bmod 3), k \geqslant k_{0}} \int\left|P_{k}^{u_{2}} \circ T_{b_{2}}^{\varphi_{2}}\left(f_{2}\right)\right|^{2}\left|Q_{k}^{0}\left(\chi_{0}(2 D) f_{0}\right)\right|^{2} \prod_{j=4}^{N}\left|P_{k}^{u_{j}} \circ T_{b_{j}}^{\varphi_{j}}\left(f_{j}\right)\right|^{2} \mathrm{~d} x\right)^{1 / 2} .
\end{aligned}
$$

Since $f_{j} \in$ bmo for $j=0$ and $j=4, \ldots, N$, arguing once again as we did for definition (53), we see that

$$
\sum_{k \geqslant k_{0}}\left|Q_{k}^{0}\left(\chi_{0}(2 D) f_{0}\right)\right|^{2} \prod_{j=4}^{N}\left|P_{k}^{u_{j}} \circ T_{b_{j}}^{\varphi_{j}}\left(f_{j}\right)\right|^{2} \mathrm{~d} x \delta_{2^{-k}}(t)
$$

is a Carleson measure, with Carleson norm bounded by $\left\|f_{0}\right\|_{\mathrm{bmo}}^{2} \prod_{j=4}^{N}\left\|f_{j}\right\|_{\mathrm{bmo}}^{2}$, and similarly,

$$
\sum_{k \geqslant k_{0}}\left|Q_{k}^{u_{1}} \circ T_{b_{1}}^{\varphi_{1}}\left(f_{1}\right)\right|^{2} \mathrm{~d} x \delta_{2^{-k}}(t)
$$

defines a Carleson measure, with Carleson norm bounded by $\left\|f_{1}\right\|_{\mathrm{bmo}}^{2}$. The $L^{2}$-boundedness of FIOs (Theorem 3.1) and the nontangential maximal function estimate (15) yield that the right-hand side of the previous inequality is bounded by

$$
\left\|f_{2}\right\|_{L^{2}} \times\left\|f_{3}\right\|_{L^{2}} \prod_{j=4}^{N}\left\|f_{j}\right\|_{\mathrm{bmo}} .
$$




\subsection{Boundedness with the target space bmo}

Here the only case to consider is the bmo $\times \cdots \times$ bmo $\rightarrow$ bmo boundedness of the operator in definition (46). In this case we take $m_{j}=-(n-1) / 2, j=0, \ldots N, f_{0} \in h^{1}$ and $f_{j} \in$ bmo for $j=1, \ldots, N$. Using definition (46) and duality, pairing against $f_{0}$, we must bound

$$
\sup _{\left\|f_{0}\right\|_{h^{1}}=1} \sum_{k \equiv \ell(\bmod 3), k \geqslant k_{0}} \int Q_{k}^{0}\left(\chi_{0}(2 D) f_{0}\right)(x)\left(Q_{k}^{u_{1}} \circ T_{b_{1}}^{\varphi_{1}}\right)(f)(x) \prod_{j=2}^{N}\left(P_{k}^{u_{j}} \circ T_{b_{j}}^{\varphi_{j}}\right)\left(f_{j}\right) \mathrm{d} x \mid .
$$

To bound this further, we apply Proposition 2.7. We take $F(x)=\chi_{0}(2 D) f_{0}(x), G(x)=T_{b_{1}}^{\varphi_{1}}\left(f_{1}\right)(x)$ and $v\left(2^{-k}, x\right)=\prod_{j=2}^{N}\left(P_{k}^{u_{j}} \circ T_{b_{j}}^{\varphi_{j}}\right)\left(f_{j}\right)(x)$. Clearly $\|F\|_{H^{1}} \lesssim\left\|f_{0}\right\|_{h^{1}}=1$, and definition (11) and Theorem 3.1 yield that $\|G\|_{\text {BMO }} \lesssim\left\|f_{1}\right\|_{\text {bmo. }}$. Applying formula (49), from Lemma 7.1 we have

$$
\left\|v\left(2^{-k}, t\right)\right\|_{L_{k, x}^{\infty}} \leqslant \prod_{j=2}^{N}\left\|P_{k}^{u_{j}} \circ T_{b_{j}}^{\varphi_{j}}\left(f_{j}\right)(x)\right\|_{L_{k, x}^{\infty}} \lesssim \prod_{j=2}^{N}\left\|f_{j}\right\|_{\mathrm{bmo}} .
$$

It follows that formula (56) is bounded by $\prod_{j=1}^{N}\left\|f_{j}\right\|_{\mathrm{bmo}}$, as required.

\section{Boundedness results for $\boldsymbol{T}_{\sigma_{j, k}}^{\Phi}$}

Our analysis of $T_{\sigma_{j, k}}^{\Phi}$ begins very similarly to that of $T_{\sigma_{j}}^{\Phi}$ in Section 7. Just as in that case, the symmetry of the operators under permutations of the frequency variables allows us to restrict our attention to just one of the $\sigma_{j, k}$, the argument for all the others being identical. We choose to study $\sigma_{1,2}$, so $\left|\xi_{1}\right|$ and $\left|\xi_{2}\right|$ are comparable to each other. More precisely, we know that

$$
c_{1} c_{3}|\Xi|^{2} \leqslant\left|\xi_{1}\right|^{2} \quad \text { and } \quad c_{1} c_{3}|\Xi|^{2} \leqslant\left|\xi_{2}\right|^{2}, \quad \text { so } \quad c_{1} c_{3}\left|\xi_{1}\right|^{2} \leqslant\left|\xi_{2}\right|^{2} \leqslant \frac{1}{c_{1} c_{3}}\left|\xi_{1}\right|^{2}
$$

on the $\Xi$-support of $\sigma_{1,2}(x, \Xi)$, with the constants $c_{1}$ and $c_{3}$ being the same as in Section 6 . We choose an integer $k_{1}$ so that $2^{-k_{1}} \leqslant c_{1} c_{3}$ and define $\widehat{\zeta}_{k}$ via

$$
\widehat{\zeta}_{k}(\xi)^{2}:=\vartheta\left(2^{-k-k_{1}-2} \xi\right)^{2}-\vartheta\left(2^{3+k_{1}-k} \xi\right)^{2}
$$

so that when $\psi_{k}\left(\xi_{1}\right) \neq 0$ and $\sigma_{1}(x, \Xi) \neq 0$,

$$
\begin{gathered}
2^{-k_{1}-2} \leqslant c_{1} c_{3}\left|2^{-k} \xi_{1}\right| \leqslant\left|2^{-k} \xi_{2}\right| \leqslant \frac{1}{c_{1} c_{3}}\left|2^{-k} \xi_{1}\right| \leqslant 2^{k_{1}+2}, \\
\text { which implies } \widehat{\zeta}_{k}\left(\xi_{2}\right)=1 .
\end{gathered}
$$

With the same choice of $\psi_{k}, \theta_{k}$ and $\chi_{0}$ as in Section 7, we can argue as we did there to write $T_{\sigma_{1,2}}^{\Phi}$ as

$$
\begin{aligned}
& T_{\sigma_{1,2}}^{\Phi}\left(f_{1}, \ldots, f_{N}\right)(x) \\
& =\int_{\mathbb{R}^{n N}} \sum_{k \geqslant k_{0}} \psi_{k}\left(\xi_{1}\right)^{2} \widehat{\zeta}_{k}\left(\xi_{2}\right)^{2} \sigma_{1,2}(x, \Xi) \chi_{0}\left(\xi_{1}\right) \widehat{f}_{1}\left(\xi_{1}\right) \times \\
& \quad \chi_{0}\left(\xi_{2}\right) \widehat{f}_{2}\left(\xi_{2}\right) \prod_{j=3}^{N} \theta_{k}\left(\xi_{j}\right)^{2} \widehat{f}_{j}\left(\xi_{j}\right) e^{i x \cdot\left(\xi_{1}+\cdots+\xi_{N}\right)+i \Phi(\Xi)} \mathrm{d} \Xi
\end{aligned}
$$

and then define

$$
\mathfrak{a}(k, x, \Xi)=\sigma_{1,2}\left(x, 2^{k} \Xi\right) \psi_{1}\left(\xi_{1}\right) \psi_{1}\left(\xi_{2}\right) \prod_{j=2}^{N} \theta_{1}\left(\xi_{j}\right)\left(\frac{2^{-k}}{\left|\xi_{1}\right|}\right)^{m-m_{2}}\left(\frac{2^{-k}}{\left|\xi_{2}\right|}\right)^{m_{2}},
$$

where once again $\sum_{j=1}^{N} m_{j}=m$, so that using definition (42), equation (57) can be rewritten as 


$$
\begin{aligned}
\sum_{k \geqslant k_{0}} & \int_{\mathbb{R}^{n N}} \mathfrak{a}\left(k, x, 2^{-k} \Xi\right) 2^{k m_{0}} \widehat{\theta_{k}}\left(\xi_{1}+\cdots+\xi_{N}\right)\left|2^{-k} \xi_{1}\right|^{m-m_{2}-m_{1}} \psi_{k}\left(\xi_{1}\right) \times \\
& \left|\xi_{1}\right|^{m_{1}} \chi_{0}\left(\xi_{1}\right) \widehat{f}_{1}\left(\xi_{1}\right) \widehat{\zeta}_{k}\left(\xi_{2}\right)\left|\xi_{2}\right|^{m_{2}} \chi_{0}\left(\xi_{2}\right) \widehat{f}_{2}\left(\xi_{2}\right) \prod_{j=3}^{N} 2^{k m_{j}} \theta_{k}\left(\xi_{j}\right)^{2} \widehat{f}_{j}\left(\xi_{j}\right) e^{i \Phi(x, \Xi) \mathrm{d} \Xi .}
\end{aligned}
$$

Just as in Section 7, the Fourier inversion formula yields

$$
\mathfrak{a}(k, x, \Xi)=\int \frac{\mathrm{m}(k, x, U)}{\left(1+|U|^{2}\right)^{M}} e^{i \Xi \cdot U} \mathrm{~d} U, \quad U=\left(u_{1}, \ldots, u_{N}\right),
$$

for a smooth bounded function $\mathrm{m}$.

So formula (58) can be written as a weighted average in $U=\left(u_{1}, \ldots, u_{N}\right)$ of

$$
\begin{gathered}
\sum_{k=k_{0}}^{\infty} \mathfrak{m}(k, x, U) \int 2^{k m_{0}} \theta_{k}\left(\xi_{1}+\cdots+\xi_{N}\right)\left[\left|2^{-k} \xi_{1}\right|^{m-m_{1}-m_{2}} \psi_{k}\left(\xi_{1}\right) e^{i 2^{-k} \xi_{1} \cdot u_{1}}\left|\xi_{1}\right|^{m_{1}} \chi_{0}\left(\xi_{1}\right) \widehat{f}_{1}\left(\xi_{1}\right)\right] \\
{\left[\widehat{\zeta}_{k}\left(\xi_{2}\right) e^{i 2^{-k} \xi_{2} \cdot u_{2}}\left|\xi_{2}\right|^{m_{2}} \chi_{0}\left(\xi_{2}\right) \widehat{f}_{2}\left(\xi_{2}\right)\right]\left[\prod_{j=3}^{N} 2^{k m_{j}} \theta_{k}\left(\xi_{j}\right) \widehat{f}_{j}\left(\xi_{j}\right) e^{i 2^{-k} \xi_{j} \cdot u_{j}}\right] e^{i \Phi(x, \Xi)} \mathrm{d} \Xi .}
\end{gathered}
$$

Therefore, we need to prove the boundedness of the operator

$$
\begin{aligned}
& D\left(f_{1}, \ldots, f_{N}\right)(x) \\
& =\sum_{k=k_{0}}^{\infty} M_{\mathfrak{m}} \circ T_{d_{0}}^{\varphi_{0}} \circ P_{k}^{0}\left[\left(Q_{k}^{u_{1}} \circ T_{d_{1}}^{\varphi_{1}}\right)\left(f_{1}\right)\left(Q_{k}^{u_{2}} \circ T_{d_{2}}^{\varphi_{2}}\right)\left(f_{2}\right) \prod_{j=3}^{N}\left(P_{k}^{u_{j}} \circ T_{d_{j}}^{\varphi_{j}}\right)\left(f_{j}\right)\right](x),
\end{aligned}
$$

where

$$
\begin{aligned}
\widehat{P_{k}^{0}(f)}(\xi) & =\theta_{k}(\xi) \widehat{f}(\xi), & d_{0}(k, \xi) & =2^{k m_{0}} \omega_{k}(\xi), \\
\widehat{Q_{k}^{u_{1}}(f)}(\xi) & =\left|2^{-k} \xi\right|^{m-m_{1}-m_{2}} \psi_{k}(\xi) e^{i 2^{-k} \xi \cdot u_{1}} \widehat{f}(\xi), & d_{1}(\xi) & =|\xi|^{m_{1}} \chi_{0}(\xi), \\
\widehat{Q_{k}^{u_{2}}(f)}(\xi) & =\psi_{k}(\xi) e^{i 2^{-k} \xi \cdot u_{2}} \widehat{f}(\xi), & d_{2}(\xi) & =|\xi|^{m_{2}} \chi_{0}(\xi), \\
\overline{P_{k}^{u_{j}}(f)}(\xi) & =\theta_{k}(\xi) e^{i 2^{-k} \xi \cdot u_{j}} \widehat{f}(\xi), & d_{j}(k, \xi) & =2^{k m_{j}} \omega_{k}(\xi),
\end{aligned}
$$

for $j=3, \ldots, N ; \omega_{k}(\xi):=\theta_{k}(\xi / 2)$ is a bump function equal to 1 on the support of $\theta_{k}$; and $M_{\mathfrak{m}}$ denotes multiplication by $\mathrm{m}^{3}$

We now proceed to consider all the necessary endpoint cases. Just as in Section 7, due to the symmetry of the form of equation (59) in the indices $j=1,2$ and $j=3, \ldots, N$, we only need to consider endpoint cases $\left(p_{0}, \ldots, p_{N}\right)$ which are distinct within the equivalence class of permutations of $\left(p_{1}, p_{2}\right)$ and $\left(p_{3}, \ldots, p_{N}\right)$. In each case, we fix

$$
\frac{1}{p_{0}}=\sum_{j=1}^{N} \frac{1}{p_{j}}, \quad 1 \leqslant p_{j} \leqslant \infty, \quad j=0, \ldots, N
$$

and

$$
m_{j}:=-(n-1)\left|\frac{1}{2}-\frac{1}{p_{j}}\right|, \quad j=0, \ldots, N,
$$

\footnotetext{
${ }^{3}$ The same ambiguity of notation arises here as in equation (45). See footnote 1.
} 
and consider $f_{j} \in X^{p_{j}}$ for $j=1, \ldots, N$. Using duality in equation (59), it is enough to estimate

$$
\sum_{k \geqslant k_{0}} \int P_{k}^{0} \circ T_{d_{0}}^{-\varphi_{0}}\left(M_{\mathfrak{m}} f_{0}\right)\left[\left(Q_{k}^{u_{1}} \circ T_{d_{1}}^{\varphi_{1}}\right)\left(f_{1}\right)\left(Q_{k}^{u_{2}} \circ T_{d_{2}}^{\varphi_{2}}\right)\left(f_{2}\right) \prod_{j=3}^{N}\left(P_{k}^{u_{j}} \circ T_{d_{j}}^{\varphi_{j}}\right)\left(f_{j}\right)\right] \mathrm{d} x,
$$

for $f_{0} \in X^{p_{0}^{\prime}}$ with $\left\|f_{0}\right\|_{X^{p_{0}^{\prime}}}=1$.

Comparing this analysis with that of $T_{\sigma_{j}}^{\Phi}$, observe that what was $Q_{k}^{0}$ (a multiplier supported on an annulus) in equation (45) has been replaced by $P_{k}^{0}$ (a multiplier supported on a ball) in equation (59). This means that our technique to remove the dependency of $M_{\mathfrak{m}}$ on $k$ will no longer be directly applicable. In the case where $p_{0}=1$ and $p_{0}=\infty$, the $k$-dependency is not problematic, and methods already introduced in Section 7 can be successfully applied again here. In the case where $p_{0}=2$, this dependency is more problematic. The possibility of replacing $\left(P_{k}^{u} \circ T_{d}^{\varphi}\right)(f)$ with $P_{k}^{u} \circ R_{k} \circ T_{\gamma}^{\varphi}(f)$ as in Lemma 7.1 is not available to us, since $m_{0}=0$, and therefore this method does not allow us to use formula (17) to estimate the $f_{0}$ term. We present an alternative approach which can successfully deal with this $k$-dependency in this case in Section 8.1.

\subsection{The endpoint cases with target space $L^{2}$}

We write

$$
P_{k}^{0}=\sum_{\ell=k_{0}+1}^{k} Q_{\ell}+P_{k_{0}}^{0}
$$

where $\widehat{Q_{\ell}(f)}(\xi)=\left(\theta_{\ell}(\xi)-\theta_{\ell-1}(\xi)\right) \widehat{f}(\xi)$, and so formula (60) is the sum of

$$
\begin{aligned}
& \sum_{k \geqslant k_{0}} \int P_{k_{0}}^{0} \circ T_{d_{0}}^{-\varphi_{0}}\left(M_{\mathrm{m}} f_{0}\right)\left[\left(Q_{k}^{u_{1}} \circ T_{d_{1}}^{\varphi_{1}}\right)\left(f_{1}\right)\left(Q_{k}^{u_{2}} \circ T_{d_{2}}^{\varphi_{2}}\right)\left(f_{2}\right) \prod_{j=3}^{N}\left(P_{k}^{u_{j}} \circ T_{d_{j}}^{\varphi_{j}}\right)\left(f_{j}\right)\right] \mathrm{d} x \\
& \lesssim\left(\sum_{k \geqslant k_{0}} \int\left|P_{k_{0}}^{0} \circ T_{d_{0}}^{-\varphi_{0}}\left(M_{\mathrm{m}} f_{0}\right)\left(Q_{k}^{u_{2}} \circ T_{d_{2}}^{\varphi_{2}}\right)\left(f_{2}\right)\right|^{2} \mathrm{~d} x\right)^{1 / 2} \\
& \quad \times\left(\sum_{k \geqslant k_{0}} \int\left|\left(Q_{k}^{u_{1}} \circ T_{d_{1}}^{\varphi_{1}}\right)\left(f_{1}\right) \prod_{j=3}^{N}\left(P_{k}^{u_{j}} \circ T_{d_{j}}^{\varphi_{j}}\right)\left(f_{j}\right)\right|^{2} \mathrm{~d} x\right)^{1 / 2}
\end{aligned}
$$

and

$$
\begin{aligned}
& \sum_{k=k_{0}}^{\infty} \sum_{\ell=k_{0}}^{k} \int Q_{\ell} \circ T_{d_{0}}^{-\varphi_{0}}\left(M_{\mathrm{m}} f_{0}\right)\left[\left(Q_{k}^{u_{1}} \circ T_{d_{1}}^{\varphi_{1}}\right)\left(f_{1}\right)\left(Q_{k}^{u_{1}} \circ T_{d_{2}}^{\varphi_{2}}\right)\left(f_{2}\right) \prod_{j=3}^{N}\left(P_{k}^{u_{j}} \circ T_{d_{j}}^{\varphi_{j}}\right)\left(f_{j}\right)\right] \mathrm{d} x \\
& =\sum_{\ell=k_{0}}^{\infty} \sum_{k=\ell}^{\infty} \int Q_{\ell} \circ T_{d_{0}}^{-\varphi_{0}}\left(M_{\mathfrak{m}} f_{0}\right)\left[\left(Q_{k}^{u_{1}} \circ T_{d_{1}}^{\varphi_{1}}\right)\left(f_{1}\right)\left(Q_{k}^{u_{1}} \circ T_{d_{2}}^{\varphi_{2}}\right)\left(f_{2}\right) \prod_{j=3}^{N}\left(P_{k}^{u_{j}} \circ T_{d_{j}}^{\varphi_{j}}\right)\left(f_{j}\right)\right] \mathrm{d} x \\
& =\sum_{\ell=k_{0}}^{\infty} \sum_{k=0}^{\infty} \int Q_{\ell} \circ T_{d_{0}}^{-\varphi_{0}}\left(M_{\mathfrak{m}} f_{0}\right)\left[\left(Q_{\ell+k}^{u_{1}} \circ T_{d_{1}}^{\varphi_{1}}\right)\left(f_{1}\right)\left(Q_{\ell+k}^{u_{1}} \circ T_{d_{2}}^{\varphi_{2}}\right)\left(f_{2}\right) \prod_{j=3}^{N}\left(P_{\ell+k}^{u_{j}} \circ T_{d_{j}}^{\varphi_{j}}\right)\left(f_{j}\right)\right] \mathrm{d} x,
\end{aligned}
$$

where we remind the reader that now in the last expression $d_{0}$ and $M_{\mathrm{m}}$ depend on $\ell+k$, and we have taken $m_{0}=0$. Given the frequency support properties on $Q_{\ell} \circ T_{d_{0}}^{-\varphi_{0}}$, we can redefine $d_{0} \equiv 1$ without changing the operator and so make it independent of $k+\ell$. Equally, the composition $Q_{\ell} \circ T_{d_{0}}^{-\varphi_{0}} \circ M_{\mathrm{m}}$ can be replaced by a finite sum of operators of the form $Q_{\ell} \circ T_{d_{0}}^{-\varphi_{0}} \circ M_{k}$, where $M_{k}$ depends only on $k$ 
(and $x$ and $U$ ), in the same way as we obtained definition (46). Thus our task is to bound

$$
\begin{aligned}
& \sum_{\ell=k_{0}}^{\infty} \int Q_{\ell} \circ T_{d_{0}}^{-\varphi_{0}}\left(M_{k} f_{0}\right)\left(Q_{k+\ell}^{u_{1}} \circ T_{d_{1}}^{\varphi_{1}}\right)\left(f_{1}\right)\left(Q_{k+\ell}^{u_{1}} \circ T_{d_{2}}^{\varphi_{2}}\right)\left(f_{2}\right) \prod_{j=3}^{N}\left(P_{k+\ell}^{u_{j}} \circ T_{d_{j}}^{\varphi_{j}}\right)\left(f_{j}\right) \mathrm{d} x \\
& \lesssim\left(\sum_{\ell \geqslant k_{0}} \int\left|Q_{\ell} \circ T_{d_{0}}^{-\varphi_{0}}\left(M_{k} f_{0}\right)\right|^{2} \mathrm{~d} x\right)^{1 / 2} \\
& \quad \times\left(\left.\sum_{\ell \geqslant k_{0}} \int\left|\left(Q_{k+\ell}^{u_{1}} \circ T_{d_{1}}^{\varphi_{1}}\right)\left(f_{1}\right)\left(Q_{k+\ell}^{u_{1}} \circ T_{d_{2}}^{\varphi_{2}}\right)\left(f_{2}\right) \prod_{j=3}^{N}\left(P_{k+\ell}^{u_{j}} \circ T_{d_{j}}^{\varphi_{j}}\right)\left(f_{j}\right)\right|^{2} \mathrm{~d} x\right|^{1 / 2}\right. \\
& \lesssim\left\|f_{0}\right\|_{L^{2}}\left(\sum_{\ell \geqslant k_{0}} \int\left|\left(Q_{k+\ell}^{u_{1}} \circ T_{d_{1}}^{\varphi_{1}}\right)\left(f_{1}\right)\left(Q_{k+\ell}^{u_{1}} \circ T_{d_{2}}^{\varphi_{2}}\right)\left(f_{2}\right) \prod_{j=3}^{N}\left(P_{k+\ell}^{u_{j}} \circ T_{d_{j}}^{\varphi_{j}}\right)\left(f_{j}\right)\right|^{2} \mathrm{~d} x\right)^{1 / 2}
\end{aligned}
$$

(where we made use of formula (17)) so that it is summable in $k$; plus we must, of course, bound formula (61).

We begin by further estimating the first factor on the right-hand side of formula (61). In each endpoint case that follows we will have $p_{2}=\infty$, so that

$$
\sum_{k \geqslant k_{0}}\left|\left(Q_{k}^{u_{2}} \circ T_{d_{2}}^{\varphi_{2}}\right)\left(f_{2}\right)(x)\right|^{2} \mathrm{~d} x \delta_{2^{-k}}(t)
$$

is always a Carleson measure, with Carleson norm bounded by $\left\|f_{2}\right\|_{\text {bmo. }}$ Observe also that Lemma 2.3 yields

$$
\left(P_{k_{0}}^{0} \circ T_{d_{0}}^{-\varphi_{0}} \circ M_{\mathrm{m}}\right)\left(f_{0}\right)=\left(P_{k_{0}}^{0} \circ T_{d_{0}}^{-\varphi_{0}} \circ P_{k}^{0} \circ M_{\mathrm{m}}\right)\left(f_{0}\right)=K *\left(\left(P_{k}^{0} \circ M_{\mathrm{m}}\right)\left(f_{0}\right)\right)
$$

for $k \geqslant k_{0}$, with $|K(\cdot)| \lesssim\langle\cdot\rangle^{-n-\varepsilon}$.

Therefore, using Minkowski's integral inequality and estimate (14), the first factor on the right-hand side of formula (61) can be controlled using the nontangential maximal function as

$$
\left\|f_{2}\right\|_{\text {bmo }} \int|K(z)|\left(\int \sup _{k \geqslant k_{0},|y-x| \leqslant 2^{-k}}\left|P_{k}^{0}\left(M_{\mathfrak{m}} f_{0}\right)(y-z)\right|^{2} \mathrm{~d} x\right)^{1 / 2} \mathrm{~d} z .
$$

However, since $P_{k}$ is convolution with a Schwartz function scaled by a factor $2^{-k}$ and $M_{\mathfrak{m}}$ is uniformly bounded, we have

$$
\sup _{k \geqslant k_{0},|y-x| \lesssim 2^{-k}}\left|P_{k}^{0}\left(M_{\mathfrak{m}} f_{0}\right)(y-z)\right| \lesssim\left|\left(M f_{0}\right)(x-z)\right|,
$$

where $M$ is the Hardy-Littlewood maximal function. Thus we have the estimate

$$
\left(\sum_{k \geqslant k_{0}} \int\left|P_{k_{0}}^{0} \circ T_{d_{0}}^{-\varphi_{0}}\left(M_{\mathrm{m}} f_{0}\right)\left(Q_{k}^{u_{2}} \circ T_{d_{2}}^{\varphi_{2}}\right)\left(f_{2}\right)\right|^{2} \mathrm{~d} x\right)^{1 / 2} \lesssim\left\|f_{0}\right\|_{L^{2}}\left\|f_{2}\right\|_{\mathrm{bmo}}
$$

for the first factor in formula (61).

We will see that to estimate formula (62) uniformly in $k$ is a similar task to what was done in Section 7. We must, however, also obtain summability in $k$. The content of the next lemma is the observation that there is some decay in the size of the Carleson norms that appear. 
Lemma 8.1. If $n \geqslant 2$ and $f \in$ bmo, then for $j=1,2$,

$$
\mathrm{d} \mu_{k}(x, t)=\sum_{k^{\prime}=0}^{\infty}\left|\left(Q_{k+k^{\prime}}^{u_{j}} \circ T_{d_{j}}^{\varphi_{j}}\right)(f)(x)\right|^{2} \delta_{2^{-k^{\prime}}}(t) \mathrm{d} x
$$

is a Carleson measure, with Carleson norm $2^{-k / 2}\|f\|_{\mathrm{bmo}}^{2}$.

Proof. For definiteness, take $j=1$. Since we can write $Q_{k+\ell}^{u_{1}} \circ T_{d_{1}}^{\varphi_{1}}=Q_{k+\ell}^{u_{1}} \circ T_{d_{1}}^{\varphi_{1}} \circ \tilde{Q}_{k+\ell}$, where $\tilde{Q}_{k+\ell}$ maps bmo into $L^{\infty}$ uniformly in $k+\ell$, as a first step we consider $f \in L^{\infty}$.

The operator $Q_{k+\ell}^{u_{1}} \circ T_{d_{1}}^{\varphi_{1}}$ is just the $(k+\ell)$ th component of the Seeger-Sogge-Stein decomposition of the Fourier integral operator $T_{d_{1}}^{\varphi_{1}}$, which we saw in Section 3.2.1. This in turn is split into $O\left(2^{(k+\ell)(n-1) / 2}\right)$ separate operators $T_{k+\ell}^{v}\left(v=1,2, \ldots, c 2^{(k+\ell)(n-1) / 2}\right)$ with kernels $K_{k+\ell}^{v}(x, y)$ which, as can be seen from formula (26), satisfy

$$
\left|K_{j}^{v}(x, y)\right| \leqslant c 2^{j}\left\{1+2^{j}\left|\left(x+\nabla \varphi_{1}\left(\xi_{j}^{v}\right)-y\right)_{1}\right|+2^{j / 2}\left|\left(x+\nabla \varphi_{1}\left(\xi_{j}^{v}\right)-y\right)^{\prime}\right|\right\}^{-\mathcal{N}},
$$

for any $\mathcal{N}>0$ and all $j \geqslant 0$. Here we have chosen a coordinate system where $x_{1}$ is parallel to $\xi_{j}^{v}$ (which was also defined in Section 3.2.1), and $x^{\prime}$ denotes the vector of remaining coordinates. For a given ball $B \subset \mathbb{R}^{n}$ with centre $\chi_{0}$ and radius $r \leqslant 1$, we write $g_{j}^{v}=f \chi_{\mathscr{R}_{j}^{v}}$ and $h_{j}^{v}=f \chi_{\left(\mathscr{R}_{j}^{v}\right)^{c}}$, with $\mathscr{R}_{j}^{v}$ being a rectangle with side length $2 r$ parallel to $\nabla \varphi_{1}\left(\xi_{j}^{v}\right)$, side length $2 r^{1 / 2}$ in the remaining directions and centre $x_{0}+\nabla \varphi_{1}\left(\xi_{j}^{v}\right)$. Clearly, then, $f=g_{j}^{v}+h_{j}^{v}$ and

$$
\left(Q_{j}^{u_{1}} \circ T_{d_{1}}^{\varphi_{1}}\right)(f)=\sum_{v} T_{j}^{v}(f)=\sum_{v} T_{j}^{v}\left(g_{j}^{v}\right)+\sum_{v} T_{j}^{v}\left(h_{j}^{v}\right) .
$$

Since $T_{j}^{v}$ are multipliers whose $L^{2}$-norms are bounded by $2^{-j(n-1) / 2}$ and whose symbols have almost disjoint support - that is, with finitely many overlaps - we have

$$
\begin{aligned}
\int_{B}\left|\sum_{v} T_{j}^{v}\left(g_{j}^{v}\right)(x)\right|^{2} \mathrm{~d} x & \leqslant \int\left|\sum_{v} T_{j}^{v}\left(g_{j}^{v}\right)(x)\right|^{2} \mathrm{~d} x \lesssim \sum_{v} \int\left|T_{j}^{v}\left(g_{j}^{v}\right)(x)\right|^{2} \mathrm{~d} x \\
& \lesssim \sum_{v} 2^{-j(n-1)} \int\left|g_{j}^{v}(x)\right|^{2} \mathrm{~d} x \lesssim \sum_{v} 2^{-j(n-1)} \int_{\mathscr{R}_{j}^{v}}|f(x)|^{2} \mathrm{~d} x \\
& \lesssim \sum_{v} 2^{-j(n-1)}\left|\mathscr{R}_{j}^{v}\right|\|f\|_{L^{\infty}}^{2} \lesssim 2^{-j(n-1) / 2} r^{-(n-1) / 2}|B|\|f\|_{L^{\infty}}^{2} .
\end{aligned}
$$

Using formula (65), we also have

$$
\begin{aligned}
& \left|T_{j}^{v}\left(h_{j}^{v}\right)(x)\right| \\
& \leqslant \int_{\left(\mathscr{R}_{j}^{v}\right)^{c}} \frac{c 2^{j} f_{1}(y)}{\left\{1+2^{j}\left|\left(x+\nabla \varphi_{1}\left(\xi_{j}^{v}\right)-y\right)_{1}\right|+2^{j / 2}\left|\left(x+\nabla \varphi_{1}\left(\xi_{j}^{v}\right)-y\right)^{\prime}\right|\right\}^{n+1}} \mathrm{~d} y \\
& \leqslant 2^{-j(n-1) / 2} \int_{\left(\mathscr{R}_{j}^{v}\right)^{c}} \frac{c 2^{j(n+1) / 2} f_{1}(y)}{\left\{1+2^{j}\left|\left(x+\nabla \varphi_{1}\left(\xi_{j}^{\nu}\right)-y\right)_{1}\right|+2^{j / 2}\left|\left(x+\nabla \varphi_{1}\left(\xi_{j}^{\nu}\right)-y\right)^{\prime}\right|\right\}^{n+1}} \mathrm{~d} y \\
& \leqslant 2^{-j(n-1) / 2}\left\|f_{1}\right\|_{L^{\infty}} \int_{\left(\mathscr{R}_{j}^{v}\right)^{c}} \frac{c 2^{j(n+1) / 2}}{\left\{1+2^{j}\left|\left(x+\nabla \varphi_{1}\left(\xi_{j}^{v}\right)-y\right)_{1}\right|+2^{j / 2}\left|\left(x+\nabla \varphi_{1}\left(\xi_{j}^{\nu}\right)-y\right)^{\prime}\right|\right\}^{n+1}} \mathrm{~d} y .
\end{aligned}
$$


For $x \in B$ and $y \in\left(\mathscr{R}_{j}^{v}\right)^{c}$, we must have either

$$
2^{j}\left|\left(x+\nabla \varphi_{1}\left(\xi_{j}^{v}\right)-y\right)_{1}\right| \geqslant 2^{j} r \quad \text { or } \quad 2^{j / 2}\left|\left(x+\nabla \varphi_{1}\left(\xi_{j}^{v}\right)-y\right)^{\prime}\right| \geqslant 2^{j / 2} r^{1 / 2} .
$$

Moreover, for those $j$ such that $2^{-j} \leqslant r$, we have $2^{j} r \geqslant 2^{j / 2} r^{1 / 2}$. Thus, for all such $j$, formula (66) yields

$$
\begin{aligned}
& 1+2^{j}\left|\left(x+\nabla \varphi_{1}\left(\xi_{j}^{v}\right)-y\right)_{1}\right|+2^{j / 2}\left|\left(x+\nabla \varphi_{1}\left(\xi_{j}^{v}\right)-y\right)^{\prime}\right| \\
& \geqslant \frac{1}{2}\left(2^{j / 2} r^{1 / 2}+2^{j}\left|\left(x+\nabla \varphi_{1}\left(\xi_{j}^{v}\right)-y\right)_{1}\right|+2^{j / 2}\left|\left(x+\nabla \varphi_{1}\left(\xi_{j}^{v}\right)-y\right)^{\prime}\right|\right) .
\end{aligned}
$$

Therefore,

$$
\begin{aligned}
& \int_{\left(\mathscr{R}_{j}^{v}\right)^{c}} \frac{c 2^{j(n+1) / 2}}{\left\{1+2^{j}\left|\left(x+\nabla \varphi_{1}\left(\xi_{j}^{v}\right)-y\right)_{1}\right|+2^{j / 2}\left|\left(x+\nabla \varphi_{1}\left(\xi_{j}^{v}\right)-y\right)^{\prime}\right|\right\}^{n+1}} \mathrm{~d} y \\
& \lesssim \int_{\left(\mathscr{R}_{j}^{v}\right)^{c}} \frac{c 2^{j(n+1) / 2}}{\left\{2^{j / 2} r^{1 / 2}+2^{j}\left|\left(x+\nabla \varphi_{1}\left(\xi_{j}^{v}\right)-y\right)_{1}\right|+2^{j / 2}\left|\left(x+\nabla \varphi_{1}\left(\xi_{j}^{v}\right)-y\right)^{\prime}\right|\right\}^{n+1}} \mathrm{~d} y \\
& \lesssim \int \frac{c 2^{j(n+1) / 2}}{\left\{2^{j / 2} r^{1 / 2}+2^{j}\left|y_{1}\right|+2^{j / 2}\left|y^{\prime}\right|\right\}^{n+1}} \mathrm{~d} y \\
& \leqslant \int \frac{c 2^{j n / 2}}{\left\{2^{j / 2} r^{1 / 2}+2^{j / 2}|y|\right\}^{n+1}} \mathrm{~d} y \\
& \leqslant \int \frac{c 2^{-j / 2}}{\left\{r^{1 / 2}+|y|\right\}^{n+1}} d y \leqslant \frac{2^{-j / 2}}{r^{1 / 2}} \int \frac{c r^{1 / 2}}{\left\{r^{1 / 2}+|y|\right\}^{n+1}} \mathrm{~d} y \lesssim \frac{2^{-j / 2}}{r^{1 / 2}} .
\end{aligned}
$$

We conclude that for $x \in B$ and $j$ such that $2^{-j} \leqslant r$,

$$
\left|T_{j}^{v}\left(h_{j}^{v}\right)(x)\right| \lesssim 2^{-j(n-1) / 2} \frac{2^{-j / 2}}{r^{1 / 2}}\|f\|_{L^{\infty}}
$$

Combining these estimates enables us to estimate

$$
\begin{aligned}
& \int_{B \times[0, r]}\left|\mathrm{d} \mu_{k}(x, t)\right|=\sum_{2^{-\ell} \leqslant r} \int_{B}\left|\left(Q_{k+\ell}^{u_{1}} \circ T_{d_{1}}^{\varphi_{1}} \circ \tilde{Q}_{k+\ell}\right)(f)\right|^{2} \mathrm{~d} x \\
& \lesssim \sum_{2^{-\ell \leqslant r}}\left(2^{-k(n-1) / 2} 2^{-\ell(n-1) / 2} r^{-(n-1) / 2}+2^{-k / 2} 2^{-\ell / 2} r^{-1 / 2}\right)|B|\left\|\tilde{Q}_{k+\ell}(f)\right\|_{L^{\infty}}^{2} \\
& \lesssim\left(2^{-k(n-1) / 2}+2^{-k / 2}\right)|B|\|f\|_{\mathrm{bmo}}^{2} \\
& \lesssim 2^{-k / 2}|B|\|f\|_{\mathrm{bmo}}^{2},
\end{aligned}
$$

for $k \geqslant 0$ and $n \geqslant 2$.

Now if the radius $r$ of $B$ is larger than 1, then we cover $B$ by balls $B_{j}$ of radius $\frac{1}{2}$, observing that there are $O\left(r^{n}\right)$ such balls $\left\{B_{j}\right\}$ needed for this covering. Furthermore, we observe that for $r>1$, 
equation (67) yields

$$
\begin{aligned}
\int_{B \times[0, r]}\left|\mathrm{d} \mu_{k}(x, t)\right| & =\int_{B \times[0,1]}\left|\mathrm{d} \mu_{k}(x, t)\right| \leqslant \sum_{O\left(r^{n}\right)} \int_{B_{j} \times[0,1]}\left|\mathrm{d} \mu_{k}(x, t)\right| \\
& \lesssim \sum_{O\left(r^{n}\right)} 2^{-k / 2} 2^{-n}\|f\|_{\mathrm{bmo}}^{2} \lesssim 2^{-k / 2}|B|\|f\|_{\mathrm{bmo}}^{2} .
\end{aligned}
$$

Thus, we have proved that $d \mu_{k}(x, \ell)$ is a Carleson measure with norm at most $2^{-k / 2}\|f\|_{\text {bmo, }}^{2}$, provided $n \geqslant 2$.

\subsection{1. bmo $\times \cdots \times$ bmo $\times L^{2} \rightarrow L^{2}$}

Here we take $n \geqslant 2, m_{j}=-(n-1) / 2, f_{j} \in$ bmo for $j=1, \ldots, N-1, m_{N}=0$ and $f_{N} \in L^{2}$.

Lemma 7.1 shows us that

$$
\sup _{k \geqslant k_{0}}\left\|\left(P_{k}^{u_{j}} \circ T_{d_{j}}^{\varphi_{j}}\right)\left(f_{j}\right)\right\|_{L^{\infty}} \lesssim\left\|f_{j}\right\|_{\mathrm{bmo}} \quad \text { for } j=3, \ldots, N \text { whenever } p_{j}=\infty .
$$

Using formulas (68) and (15) and Theorem 3.1, we can estimate

$$
\begin{aligned}
& \left(\sum_{k \geqslant k_{0}} \int\left|\left(Q_{k}^{u_{1}} \circ T_{d_{1}}^{\varphi_{1}}\right)\left(f_{1}\right) \prod_{j=3}^{N}\left(P_{k}^{u_{j}} \circ T_{d_{j}}^{\varphi_{j}}\right)\left(f_{j}\right)\right|^{2} \mathrm{~d} x\right)^{1 / 2} \\
& \lesssim\left\|f_{1}\right\|_{\mathrm{bmo}} \prod_{j=3}^{N-1}\left\|f_{j}\right\|_{\mathrm{bmo}}\left\|f_{N}\right\|_{L^{2}},
\end{aligned}
$$

and combining this with formula (64) bounds formula (61), as required.

To bound formula (62), we see from Lemma 8.1 and formula (68) that

$$
\sum_{k \geqslant k_{0}}\left|\left(Q_{k+\ell}^{u_{1}} \circ T_{d_{1}}^{\varphi_{1}}\right)\left(f_{1}\right)\left(Q_{k+\ell}^{u_{2}} \circ T_{d_{2}}^{\varphi_{2}}\right)\left(f_{2}\right) \prod_{j=3}^{N-1}\left(P_{k}^{u_{j}} \circ T_{d_{j}}^{\varphi_{j}}\right)\left(f_{j}\right)\right|^{2} \mathrm{~d} x \delta_{2^{-k}}(t)
$$

is a Carleson measure, with norm $2^{k / 2} \prod_{j=2}^{N-1}\left\|f_{j}\right\|_{\mathrm{bmo}}^{2}$. Therefore, again by formula (15) and Theorem 3.1, we see that formula (62) is bounded by $2^{k / 4}\left\|f_{0}\right\|_{L^{2}} \prod_{j=1}^{N-1}\left\|f_{j}\right\|_{\mathrm{bmo}}\left\|f_{N}\right\|_{L^{2}}$, which again is sufficient for our purposes.

\subsection{2. $L^{2} \times$ bmo $\times \cdots \times$ bmo $\rightarrow L^{2}$}

Here we take $m_{1}=0, f_{1} \in L^{2}, m_{j}=-(n-1) / 2$ and $f_{j} \in$ bmo for $j=2, \ldots, N$.

Using formulas (68) and (17) and Theorem 3.1, we can estimate

$$
\left(\sum_{k \geqslant k_{0}} \int\left|\left(Q_{k}^{u_{1}} \circ T_{d_{1}}^{\varphi_{1}}\right)\left(f_{1}\right) \prod_{j=3}^{N}\left(P_{k}^{u_{j}} \circ T_{d_{j}}^{\varphi_{j}}\right)\left(f_{j}\right)\right|^{2} \mathrm{~d} x\right)^{1 / 2} \lesssim\left\|f_{0}\right\|_{L^{2}}\left\|f_{1}\right\|_{L^{2}} \prod_{j=3}^{N}\left\|f_{j}\right\|_{\mathrm{bmo}},
$$

and combining this with formula (64) bounds formula (61), as required.

To bound formula (62), we see from Lemma 8.1 and formula (68) that

$$
\sum_{\substack{k \geqslant k_{0} \\ \mid}}\left|\left(Q_{k+\ell}^{u_{2}} \circ T_{d_{2}}^{\varphi_{2}}\right)\left(f_{2}\right) \prod_{j=3}^{N}\left(P_{k}^{u_{j}} \circ T_{d_{j}}^{\varphi_{j}}\right)\left(f_{j}\right)\right|^{2} \mathrm{~d} x \delta_{2^{-k}}(t)
$$


is a Carleson measure, with norm $2^{k / 2} \prod_{j=2}^{N}\left\|f_{j}\right\|_{\text {bmo }}^{2}$. Therefore, by formula (15) and Theorem 3.1, we see that formula (62) is bounded by $2^{k / 4}\left\|f_{0}\right\|_{L^{2}}\left\|f_{1}\right\|_{L^{2}} \prod_{j=2}^{N}\left\|f_{j}\right\|_{\text {bmo }}$, which is sufficient to conclude the proof of this endpoint case.

\subsection{The endpoint cases with target space $h^{1}$}

The operator $M_{\mathfrak{m}}$ (which we recall depends on $k$ ) can be viewed as a pseudodifferential operator, and therefore (see [11]),

$$
\left\|M_{\mathfrak{m}}\left(f_{0}\right)\right\|_{\text {bmo }} \lesssim \sum_{|\alpha| \lesssim 1}\left\|\partial^{\alpha} \mathfrak{m}\right\|_{L^{\infty}}\left\|f_{0}\right\|_{\text {bmo }} \lesssim\left\|f_{0}\right\|_{\text {bmo }},
$$

with implicit constants independent of $k$. Thus Lemma 7.1 yields

$$
\sup _{k \geqslant k_{0}}\left\|P_{k}^{0} \circ T_{d_{0}}^{-\varphi_{0}}\left(M_{\mathrm{m}} f_{0}\right)\right\|_{L^{\infty}} \lesssim\left\|f_{0}\right\|_{\mathrm{bmo}} \lesssim 1
$$

Moreover, as a scholium to Lemma 7.1, we have

$$
\sup _{k \geqslant k_{0}}\left\|\left(Q_{k}^{u_{j}} \circ T_{d_{j}}^{\varphi_{j}}\right)\left(f_{j}\right)\right\|_{L^{\infty}} \lesssim\left\|f_{j}\right\|_{\mathrm{bmo}} \quad \text { for } j=1,2 \text { when } p_{j}=\infty \text {. }
$$

\subsection{1. bmo $\times \cdots \times$ bmo $\times h^{1} \rightarrow h^{1}$}

Here we take $m_{j}=-(n-1) / 2$ for $j=0, \ldots, N, f_{j} \in$ bmo for $j=0, \ldots, N-1$ and $f_{N} \in h^{1}$.

By formulas (68) and (69), we see that

$$
\begin{aligned}
\mathrm{d} \mu(x, t) & :=\sum_{k \geqslant k_{0}} P_{k}^{0} \circ T_{d_{0}}^{-\varphi_{0}}\left(M_{\mathfrak{m}} f_{0}\right) \\
& \times\left[\left(Q_{k}^{u_{1}} \circ T_{d_{1}}^{\varphi_{1}}\right)\left(f_{1}\right)\left(Q_{k}^{u_{1}} \circ T_{d_{2}}^{\varphi_{2}}\right)\left(f_{2}\right) \prod_{j=3}^{N-1}\left(P_{k}^{u_{j}} \circ T_{d_{j}}^{\varphi_{j}}\right)\left(f_{j}\right)\right] \mathrm{d} x \delta_{2^{-k}}(t)
\end{aligned}
$$

defines a Carleson measure, with Carleson norm bounded by

$$
\left\|f_{0}\right\|_{\mathrm{bmo}} \prod_{j \neq j_{0}}\left\|f_{j}\right\|_{\mathrm{bmo}}\left\|f_{1}\right\|_{\mathrm{bmo}}\left\|f_{2}\right\|_{\mathrm{bmo}} \text {. }
$$

So formula (60) becomes

$$
\sum_{k \geqslant k_{0}} \int P_{k}^{u_{N}} T_{d_{N}}^{\varphi_{N}}\left(f_{N}\right)(x) \mathrm{d} \mu\left(x, 2^{-k}\right)
$$

and arguing as in Section 7.2.1, it follows that

$$
\left|\sum_{k \geqslant k_{0}} \int P_{k}^{u_{N}} \circ T_{d_{N}}^{\varphi_{N}}\left(f_{N}\right)(x) \mathrm{d} \mu\left(x, 2^{-k}\right)\right| \lesssim \prod_{j=0}^{N-1}\left\|f_{j}\right\|_{\mathrm{bmo}}\left\|f_{N}\right\|_{h^{1}} .
$$

\subsection{2. $h^{1} \times$ bmo $\times \cdots \times$ bmo $\rightarrow h^{1}$}

Here we take $m_{j}=-(n-1) / 2$ for $j=0, \ldots, N, f_{0} \in$ bmo, $f_{1} \in h^{1}$ and $f_{j} \in$ bmo for $j=2, \ldots, N$.

Using estimates (68) and (69) again together with Proposition 2.7, Theorem 3.1 and definition (11), we can estimate formula (60) by 


$$
\begin{aligned}
& \left\|f_{0}\right\|_{\mathrm{bmo}} \prod_{j=3}^{N}\left\|f_{j}\right\|_{\mathrm{bmo}}\left\|T_{d_{1}}^{\varphi_{1}}\left(f_{1}\right)\right\|_{H^{1}}\left\|T_{d_{2}}^{\varphi_{2}}\left(f_{2}\right)\right\|_{\mathrm{BMO}} \\
& \leqslant\left\|f_{0}\right\|_{\mathrm{bmo}} \prod_{j=3}^{N}\left\|f_{j}\right\|_{\mathrm{bmo}}\left\|f_{1}\right\|_{h^{1}}\left\|f_{2}\right\|_{\mathrm{bmo}} .
\end{aligned}
$$

\subsection{3. $L^{2} \times L^{2} \times$ bmo $\times \cdots \times$ bmo $\rightarrow h^{1}$}

We choose $m_{1}=m_{2}=0, f_{1}, f_{2} \in L^{2}, m_{j}=-\frac{n-1}{2}$ and $f_{j} \in$ bmo for $j=3, \ldots N$.

Once again, estimates (68) and (69) and Theorem 3.1, this time together with formula (17), mean we can estimate formula (60) by

$$
\begin{aligned}
& \sum_{k \geqslant k_{0}} \int\left|\left(Q_{k}^{u_{1}} \circ T_{d_{1}}^{\varphi_{1}}\right)\left(f_{1}\right)\left(Q_{k}^{u_{2}} \circ T_{d_{2}}^{\varphi_{2}}\right)\left(f_{2}\right)\right| \mathrm{d} x \prod_{j=3}^{N}\left\|f_{j}\right\|_{\mathrm{bmo}} \\
& \lesssim\left(\sum_{k \geqslant k_{0}} \int\left|\left(Q_{k}^{u_{1}} \circ T_{d_{1}}^{\varphi_{1}}\right)\left(f_{1}\right)\right|^{2} \mathrm{~d} x\right)^{1 / 2}\left(\sum_{k \geqslant k_{0}} \int\left|\left(Q_{k}^{u_{2}} \circ T_{d_{2}}^{\varphi_{2}}\right)\left(f_{2}\right)\right|^{2} \mathrm{~d} x\right)^{1 / 2} \prod_{j=3}^{N}\left\|f_{j}\right\|_{\mathrm{bmo}} \\
& \lesssim\left\|f_{1}\right\|_{L^{2}}\left\|f_{2}\right\|_{L^{2}} \prod_{j=3}^{N}\left\|f_{j}\right\|_{\mathrm{bmo}},
\end{aligned}
$$

where we have also used the Cauchy-Schwarz inequality and quadratic estimates.

\subsection{4. bmo $\times$ bmo $\times \cdots \times$ bmo $\times L^{2} \times L^{2} \rightarrow h^{1}$}

We choose $m_{j}=-\frac{n-1}{2}$ and $f_{j} \in$ bmo for $j=1, \ldots, N-2$, and $m_{j}=0, f_{j} \in L^{2}$ for $j=N, N-1$.

Via estimates (68) and (69),

$$
\sum_{k \geqslant k_{0}} P_{k}^{0} \circ T_{d_{0}}^{-\varphi_{0}}\left(M_{\mathfrak{m}} f_{0}\right)\left[\left(Q_{k}^{u_{1}} \circ T_{d_{1}}^{\varphi_{1}}\right)\left(f_{1}\right)\left(Q_{k}^{u_{2}} \circ T_{d_{2}}^{\varphi_{2}}\right)\left(f_{2}\right)\right] \prod_{j=3}^{N-2}\left(P_{k}^{u_{j}} \circ T_{d_{j}}^{\varphi_{j}}\right)\left(f_{j}\right) \delta_{2^{-k}}(t) \mathrm{d} x
$$

can be seen to be a Carleson measure. Since $d_{j}$ is independent of $k$ when $m_{j}=0$ for $j=N$ or $N-1$, formula (15) together with Theorem 3.1 can be used to estimate formula (60) by

$$
\prod_{j=1}^{N-2}\left\|f_{j}\right\|_{\mathrm{bmo}}\left\|T_{d_{N-1}}^{\varphi_{N-1}}\left(f_{N-1}\right)\right\|_{L^{2}}\left\|T_{d_{N}}^{\varphi_{N}}\left(f_{N}\right)\right\|_{L^{2}} \lesssim \prod_{j=1}^{N-2}\left\|f_{j}\right\|_{\mathrm{bmo}}\left\|f_{N-1}\right\|_{L^{2}}\left\|f_{N}\right\|_{L^{2}} .
$$

\subsection{5. $L^{2} \times$ bmo $\times$ bmo $\times \cdots \times$ bmo $\times L^{2} \rightarrow h^{1}$}

We choose $m_{1}=m_{N}=0, f_{1}, f_{N} \in L^{2}, m_{j}=-\frac{n-1}{2}$ and $f_{j} \in$ bmo for $j=2, \ldots, N-1$.

This time we again first apply estimates (68) and (69) to formula (60), but then the Cauchy-Schwarz inequality, to obtain the estimate

$$
\begin{aligned}
& \left\|f_{0}\right\|_{\mathrm{bmo}} \prod_{j=3}^{N-1}\left\|f_{j}\right\|_{\mathrm{bmo}}\left(\sum_{k \geqslant k_{0}} \int\left|\left(Q_{k}^{u_{2}} \circ T_{d_{2}}^{\varphi_{2}}\right)\left(f_{2}\right)\left(P_{k}^{u_{N}} \circ T_{d_{N}}^{\varphi_{N}}\right)\left(f_{N}\right)\right|^{2} \mathrm{~d} x\right)^{1 / 2} \\
& \quad \times\left(\sum_{k \geqslant k_{0}} \int\left|\left(Q_{k}^{u_{1}} \circ T_{d_{1}}^{\varphi_{1}}\right)\left(f_{1}\right)\right|^{2} \mathrm{~d} x\right)^{1 / 2} .
\end{aligned}
$$


Thereafter, formulas (15) and (17) and Theorem 3.1 lead us to the bound

$$
\left\|f_{0}\right\|_{\mathrm{bmo}}\left\|f_{1}\right\|_{L^{2}} \prod_{j=2}^{N-1}\left\|f_{j}\right\|_{\mathrm{bmo}}\left\|f_{N}\right\|_{L^{2}}
$$

\subsection{The endpoint case with target space bmo}

Here we take $m_{j}=-(n-1) / 2$ and $f_{j} \in$ bmo for $j=1, \ldots, N$.

Just as we did in the proof of Lemma 7.1, and with the same notation, we write

$$
\begin{aligned}
P_{k}^{0} \circ T_{d_{0}}^{-\varphi_{0}} & =P_{k}^{0} \circ T_{d_{0}^{\mathrm{b}}}^{-\varphi_{0}}+P_{k}^{0} \circ T_{d_{0}^{\sharp}}^{-\varphi_{0}} \\
& =P_{k}^{0} \circ T_{d_{0}^{\mathrm{b}}}^{-\varphi_{0}}+\sum_{j=k_{0}}^{k} 2^{(k-j) m_{0}} Q_{j} \circ T_{\gamma}^{-\varphi_{0}}
\end{aligned}
$$

with the help of equation (51).

To estimate the term arising from the sum in $j$ in equation (71), we argue as in Section 8.1 and are led to the expression

$$
\sum_{k=0}^{\infty} 2^{k m_{0}} \sum_{\ell=k_{0}}^{\infty} \int Q_{\ell} \circ T_{\gamma}^{-\varphi_{0}}\left(M_{k} f_{0}\right)\left(Q_{k+\ell}^{u_{1}} \circ T_{d_{1}}^{\varphi_{1}}\right)\left(f_{1}\right)\left(Q_{k+\ell}^{u_{1}} \circ T_{d_{2}}^{\varphi_{2}}\right)\left(f_{2}\right) \prod_{j=3}^{N}\left(P_{k+\ell}^{u_{j}} \circ T_{d_{j}}^{\varphi_{j}}\right)\left(f_{j}\right) \mathrm{d} x
$$

The sum in $\ell$ can be estimated using formula (68), the fact (from Lemma 8.1) that

$$
\sum_{\ell \geqslant k_{0}}\left|\left(Q_{k+\ell}^{u_{1}} \circ T_{d_{1}}^{\varphi_{1}}\right)\left(f_{1}\right)(x)\left(Q_{k+\ell}^{u_{1}} \circ T_{d_{2}}^{\varphi_{2}}\right)\left(f_{2}\right)(x)\right| \mathrm{d} x \delta_{2^{-\ell}}(t)
$$

is a Carleson measure with Carleson norm of size $2^{-k / 2}\left\|f_{1}\right\|_{\mathrm{bmo}}\left\|f_{2}\right\|_{\mathrm{bmo}}$ and formula (16). It is then straightforward to sum in $k$.

To deal with the first term of the right-hand side of equation (71), we write

$$
\begin{aligned}
P_{k}^{0} \circ T_{d_{0}^{\mathrm{b}}}^{-\varphi_{0}} \circ M_{\mathfrak{m}} & =T_{d_{0}^{\mathrm{b}}}^{-\varphi_{0}} \circ P_{k}^{0} \circ M_{\mathfrak{m}} \\
& =T_{d_{0}^{\mathrm{b}}}^{-\varphi_{0}} \circ\left[P_{k}^{0}, M_{\mathfrak{m}}\right]+T_{d_{0}^{\mathrm{b}}}^{-\varphi_{0}} \circ M_{\mathfrak{m}} \circ P_{k}^{0} .
\end{aligned}
$$

A fairly standard calculation shows that the kernel of $\left[P_{k}^{0}, M_{\mathrm{m}}\right]$ is integrable and of size $2^{-k}$. This, combined with the estimate of the kernel of $T_{d_{0}^{b}}^{-\varphi_{0}}$ from Lemma 2.3, shows that

$$
\left\|T_{d_{0}^{\mathrm{b}}}^{-\varphi_{0}} \circ\left[P_{k}^{0}, M_{\mathrm{m}}\right]\left(f_{0}\right)\right\|_{L^{1}} \lesssim 2^{-k}\left\|f_{0}\right\|_{L^{1}} \lesssim 2^{-k}\left\|f_{0}\right\|_{h^{1}},
$$

which, together with formulas (68) and (70), proves

$$
\begin{aligned}
& \sum_{k=k_{0}}^{\infty} \int T_{d_{0}^{\mathrm{b}}}^{-\varphi_{0}} \circ\left[P_{k}^{0}, M_{\mathrm{m}}\right]\left(f_{0}\right)\left(Q_{k}^{u_{1}} \circ T_{d_{1}}^{\varphi_{1}}\right)\left(f_{1}\right)\left(Q_{k}^{u_{1}} \circ T_{d_{2}}^{\varphi_{2}}\right)\left(f_{2}\right) \prod_{j=3}^{N}\left(P_{k}^{u_{j}} \circ T_{d_{j}}^{\varphi_{j}}\right)\left(f_{j}\right) \mathrm{d} x \\
& \lesssim\left\|f_{0}\right\|_{h^{1}} \prod_{j=1}^{N}\left\|f_{j}\right\|_{\mathrm{bmo}} .
\end{aligned}
$$


Finally, the term associated with $T_{d_{0}^{b}}^{-\varphi_{0}} \circ M_{\mathfrak{m}} \circ P_{k}^{0}$ can be dealt with by first writing

$$
\begin{aligned}
& \sum_{k=k_{0}}^{\infty} \int T_{d_{0}^{\mathrm{b}}}^{-\varphi_{0}} \circ M_{\mathfrak{m}} \circ P_{k}^{0}\left(f_{0}\right)\left(Q_{k}^{u_{1}} \circ T_{d_{1}}^{\varphi_{1}}\right)\left(f_{1}\right)\left(Q_{k}^{u_{1}} \circ T_{d_{2}}^{\varphi_{2}}\right)\left(f_{2}\right) \prod_{j=3}^{N}\left(P_{k}^{u_{j}} \circ T_{d_{j}}^{\varphi_{j}}\right)\left(f_{j}\right) \mathrm{d} x \\
& =\sum_{k=k_{0}}^{\infty} \int M_{\mathfrak{m}} \circ P_{k}^{0}\left(f_{0}\right) T_{d_{0}^{\mathrm{b}}}^{\varphi_{0}}\left(\left(Q_{k}^{u_{1}} \circ T_{d_{1}}^{\varphi_{1}}\right)\left(f_{1}\right)\left(Q_{k}^{u_{1}} \circ T_{d_{2}}^{\varphi_{2}}\right)\left(f_{2}\right) \prod_{j=3}^{N}\left(P_{k}^{u_{j}} \circ T_{d_{j}}^{\varphi_{j}}\right)\left(f_{j}\right)\right) \mathrm{d} x .
\end{aligned}
$$

The kernel estimate of $T_{d_{0}^{b}}^{-\varphi_{0}}$ from Lemma 2.3 shows that since

$$
\sum_{\ell \geqslant k_{0}}\left|\left(Q_{k}^{u_{1}} \circ T_{d_{1}}^{\varphi_{1}}\right)\left(f_{1}\right)\left(Q_{k}^{u_{1}} \circ T_{d_{2}}^{\varphi_{2}}\right)\left(f_{2}\right) \prod_{j=3}^{N}\left(P_{k}^{u_{j}} \circ T_{d_{j}}^{\varphi_{j}}\right)\left(f_{j}\right)\right| \mathrm{d} x \delta_{2^{-\ell}}(t)
$$

is a Carleson measure, then even

$$
\sum_{\ell \geqslant k_{0}}\left|T_{d_{0}^{\varphi_{0}}}^{\varphi_{0}}\left(\left(Q_{k}^{u_{1}} \circ T_{d_{1}}^{\varphi_{1}}\right)\left(f_{1}\right)\left(Q_{k}^{u_{1}} \circ T_{d_{2}}^{\varphi_{2}}\right)\left(f_{2}\right) \prod_{j=3}^{N}\left(P_{k}^{u_{j}} \circ T_{d_{j}}^{\varphi_{j}}\right)\left(f_{j}\right)\right)\right| \mathrm{d} x \delta_{2^{-\ell}}(t)
$$

is a Carleson measure. Therefore, applying the uniform bound of $m$ and formula (16) in equation (72) completes the proof.

\section{Boundedness results for $T_{\sigma_{0}}^{\Phi}$}

For the case of $T_{\sigma_{0}}^{\Phi}$ given by

$$
T_{\sigma_{0}}^{\Phi}\left(f_{1}, \ldots, f_{N}\right)(x)=\int_{\mathbb{R}^{n N}} \sigma_{0}(x, \Xi) \prod_{j=1}^{N}\left(\widehat{f}_{j}\left(\xi_{j}\right) e^{i x \cdot \xi_{j}}\right) e^{i \Phi(\Xi)} \mathrm{d} \Xi,
$$

we use a separation-of-variables technique as follows.

Let $Q$ be a closed cube in $\mathbb{R}^{n N}$ of side length $L$ which compactly contains the $\Xi$-support of $\sigma_{0}$. We extend $\left.\sigma_{0}(x, \Xi)\right|_{\Xi \in Q}$ periodically in the $\Xi$-variables with period $L$ to $\widetilde{\sigma_{0}}(x, \Xi) \in \mathcal{C}^{\infty}\left(\mathbb{R}_{x}^{n} \times \mathbb{R}_{\Xi}^{n N}\right)$. Set $\zeta \in \mathcal{C}_{c}^{\infty}\left(\mathbb{R}^{n N}\right)$ with $\operatorname{supp} \zeta \subset Q$ and $\zeta=1$ on the $\Xi$-support of $\sigma_{0}(x, \Xi)$, so that we have $\sigma_{0}(x, \Xi)=\widetilde{\sigma_{0}}(x, \Xi) \zeta(\Xi)$. We can then find the Fourier series coefficients of $\widetilde{\sigma}(x, \Xi)$ :

$$
\begin{aligned}
a_{K}(x) & =\widetilde{\widetilde{\sigma_{0}}}(x, \Xi)(x, K)=\frac{1}{L^{n}} \int_{Q} e^{-i \frac{2 \pi}{L} \Xi \cdot K} \widetilde{\sigma_{0}}(x, \Xi) \mathrm{d} \Xi \\
& =\frac{1}{L^{n}} \int_{\mathbb{R}^{n N}} e^{-i \frac{2 \pi}{L} \Xi \cdot K} \sigma_{0}(x, \Xi) \mathrm{d} \Xi
\end{aligned}
$$

where $\Xi=\left(\xi_{1}, \ldots, \xi_{N}\right) \in \mathbb{R}^{n N}, K=\left(k_{1}, \ldots, k_{N}\right) \in \mathbb{R}^{n N}$ and $\Xi \cdot K=\sum_{j=1}^{N} \xi_{j} \cdot k_{j}=\sum_{j=1}^{N} \sum_{\ell=1}^{n} \xi_{j}^{\ell} k_{j}^{\ell}$. Also observe that using this notation, we have $\left|k_{j}\right|^{2}=\sum_{\ell=1}^{n}\left(k_{j}^{\ell}\right)^{2}$. Integration by parts then yields

$$
\left|\partial^{\alpha} a_{K}(x)\right|=\frac{c_{n, M, L}}{\left|k_{j}^{\ell}\right|^{2 M}}\left|\int e^{-i \frac{2 \pi}{L} \Xi \cdot K} \partial_{\xi_{j}^{\ell}}^{2 M} \partial_{x}^{\alpha} \sigma_{0}(x, \Xi) \mathrm{d} \Xi\right|
$$


for all multi-indices $\alpha$, any $M \geqslant 0$ and some constants $c_{n, M, L}$. Therefore, the boundedness of the $\Xi$-support of $\sigma_{0}(x, \Xi)$ and the fact that $\left|\partial^{\alpha} a_{K}(x)\right| \lesssim 1$ imply

$$
\left|\partial^{\alpha} a_{K}(x)\right| \lesssim\left(1+\sum_{j=1}^{N}\left|k_{j}\right|^{2}\right)^{-M}
$$

for all $x \in \mathbb{R}^{n}$ and $M \geqslant 0$.

We now choose $\theta \in \mathcal{C}_{c}^{\infty}\left(\mathbb{R}^{n}\right)$ such that $1=\prod_{j=1}^{N} \theta\left(\xi_{j}\right)$ for $\Xi=\left(\xi_{1}, \ldots, \xi_{N}\right)$ on the support of $\zeta$. We then even have

$$
1=\theta\left(\left(\xi_{1}+\cdots+\xi_{N}\right) / \sqrt{N}\right) \prod_{j=1}^{N} \theta\left(\xi_{j}\right)
$$

for $\Xi=\left(\xi_{1}, \ldots, \xi_{N}\right)$ on the support of $\zeta$. Using the Fourier expansion of $\widetilde{\sigma_{0}}(x, \Xi)$, we can write

$$
T_{\sigma_{0}}^{\Phi}\left(f_{1}, \ldots, f_{N}\right)(x)=\sum_{K \in \mathbb{Z}^{n N}} a_{K}(x) T_{\theta(\cdot / \sqrt{N})}^{\varphi_{0}}\left(\prod_{j=1}^{N} T_{\theta}^{\varphi_{j}} \circ \frac{\tau_{2 \pi k_{j}}}{L}\left(f_{j}\right)\right)(x),
$$

where $\tau_{h} f(x):=f(x-h)$.

Since we only need to consider the endpoint cases of Corollary 5.2, the analysis is confined to the spaces $h^{1}, L^{2}$ and bmo. Now observe that since $\theta \in \mathcal{C}_{c}^{\infty}\left(\mathbb{R}^{n}\right)$, Lemma 2.3 and formulas (10) and (11) yield

$$
\left\|T_{\theta}^{\varphi_{j}}(f)\right\|_{L^{p}} \lesssim\|f\|_{X^{p}} \quad \text { and } \quad\left\|T_{\theta(\cdot / \sqrt{N})}^{\varphi_{0}}(f)\right\|_{X^{p}} \lesssim\|f\|_{L^{p}}
$$

for $p=1,2, \infty$. Combining these estimates with the translation invariance of the norms and Hölder's inequality gives

$$
\left\|T_{\theta(\cdot / \sqrt{N})}^{\varphi_{0}}\left(\prod_{j=1}^{N} T_{\theta}^{\varphi_{j}} \circ \frac{\tau_{2 \pi k_{j}}}{L}\left(f_{j}\right)\right)\right\|_{X^{p_{0}}} \lesssim \prod_{j=1}^{N}\left\|f_{j}\right\|_{X^{p_{j}}}
$$

for all the endpoint cases of $p_{0}, p_{1}, \ldots, p_{N}$ in Corollary 5.2. Finally, the boundedness of $T_{\sigma_{0}}^{\varphi_{0}}$ follows by applying formula (73) with the inclusions $\mathrm{C}_{b}^{1} \cdot h^{1} \subseteq h^{1}, L^{\infty} \cdot L^{2} \subseteq L^{2}$ and $\mathrm{C}_{b}^{1} \cdot$ bmo $\subseteq$ bmo (see [11]).

Acknowledgements. The first author was partially supported by the Spanish Government grant MTM2016-75196-P. The second author was partially supported by the Research School in Interdisciplinary Mathematics at Linköping University. The third author was partially supported by a grant from the Holger and Anna Crafoord foundation. The authors are grateful to the referees for their useful comments and helpful suggestions.

Conflict of Interest: The authors have no conflicts of interest to declare.

\section{References}

[1] F. Bernicot and P. Germain, 'Bilinear oscillatory integrals and boundedness for new bilinear multipliers', Adv. Math. 225(4) (2010), 1739-1785.

[2] F. Bernicot and P. Germain, 'Bilinear dispersive estimates via space-time resonances I: The one-dimensional case', Anal. PDE 6(3) (2013), 687-722.

[3] F. Bernicot and P. Germain, 'Bilinear dispersive estimates via space time resonances, dimensions two and three', Arch. Ration. Mech. Anal. 214(2) (2014), 617-669.

[4] L. Carleson, 'An interpolation problem for bounded analytic functions', Amer. J. Math. 80 (1958), 921-930.

[5] R. Coifman and Y. Meyer, Au delà des opérateurs pseudo-différentiels, Astérisque, 57 (Société Mathématique de France, Paris, 1978).

[6] D. Dos Santos Ferreira and W. Staubach, Global and Local Regularity for Fourier Integral Operators on Weighted and Unweighted Spaces, Mem. Amer. Math. Soc., 229(1074) (American Mathematical Society, Providence, RI, 2014). 
[7] C. Fefferman, 'A note on spherical summation multipliers', Israel J. Math. 15 (1973), 44-52.

[8] P. Germain, N. Masmoudi, and J. Shatah, 'Global solutions for 3D quadratic Schrödinger equations', Int. Math. Res. Not. IMRN 3 (2009), 414-432.

[9] P. Germain, N. Masmoudi and J. Shatah, 'Global solutions for the gravity water waves equation in dimension 3', C. R. Math. Acad. Sci. Paris 347(15-6) (2009), 897-902 (in English, with English and French summaries).

[10] P. Germain, N. Masmoudi and J. Shatah, 'Global solutions for 2D quadratic Schrödinger equations', J. Math. Pures Appl. (9) 97(5) (2012), 505-543 (in English, with English and French summaries).

[11] D. Goldberg, 'A local version of real Hardy spaces', Duke Math. J. 46(1) (1979), 27-42.

[12] L. Grafakos, Classical Fourier Analysis, third edn, Graduate Texts in Mathematics, 249 (Springer, New York, 2014).

[13] L. Grafakos and M. Mastyło, 'Analytic families of multilinear operators', Nonlinear Anal. 107 (2014), 47-62.

[14] A. Hassell, P. Portal and J. Rozendaal, 'Off-singularity bounds and Hardy spaces for Fourier integral operators', Trans. Amer. Math. Soc. 373 (2020), 5773-5832.

[15] A. Israelsson, S. Rodríguez-López and W. Staubach, 'Local and global estimates for hyperbolic equations in Besov-Lipschitz and Triebel-Lizorkin spaces' (2019). To appear in Analysis \& PDE.

[16] S. G. Krantz, 'Fractional integration on Hardy spaces', Studia Math. 73(2) (1982), 87-94.

[17] M. Mendez and M. Mitrea, 'The Banach envelopes of Besov and Triebel-Lizorkin spaces and applications to partial differential equations', J. Fourier Anal. Appl. 6(5) (2000), 503-531.

[18] A. Miyachi, 'On some singular Fourier multipliers', J. Fac. Sci. Univ. Tokyo Sect. IA Math. 28(2) (1981), $267-315$.

[19] J. Peetre, 'On spaces of Triebel-Lizorkin type', Ark. Mat. 13 (1975), 123-130.

[20] M. M. Peloso and S. Secco 'Boundedness of Fourier integral operators on Hardy spaces', Proc. Edinb. Math. Soc. (2) 51(2) (2008), 443-463.

[21] S. Rodríguez-López, D. Rule and W. Staubach, 'A Seeger-Sogge-Stein theorem for bilinear Fourier integral operators', $A d v$. Math. 264 (2014), 1-54.

[22] M. Ruzhansky and M. Sugimoto, 'A local-to-global boundedness argument and Fourier integral operators', J. Math. Anal. Appl. 473(2) (2019), 892-904.

[23] A. Seeger, C. D. Sogge and E. M. Stein, 'Regularity properties of Fourier integral operators', Ann. of Math. (2) 134(2) (1991), 231-251.

[24] E. M. Stein, Harmonic Analysis: Real-Variable Methods, Orthogonality, and Oscillatory Integrals, Princeton Mathematical Series, 43 (Princeton University Press, Princeton, NJ, 1993). With the assistance of Timothy S. Murphy; Monographs in Harmonic Analysis, III.

[25] E. M. Stein and G. Weiss, Introduction to Fourier Analysis on Euclidean Spaces, Princeton Mathematical Series, 32 (Princeton University Press, Princeton, NJ, 1971).

[26] H. Triebel, Theory of Function Spaces, Monographs in Mathematics, 78 (Birkhäuser Verlag, Basel, 1983). 